\title{
Model-based Dynamic Resource Management for Multi Tier Information Systems
}

\author{
by \\ TAO ZHENG \\ A thesis submitted in partial fulfillment of the \\ requirements for the degree of

\section{Doctor of Philosophy} \\ Ottawa-Carleton Institute of Electrical Engineering \\ Faculty of Engineering \\ Department of Systems and Computer Engineering \\ Carleton University \\ Ottawa, Ontario, Canada, K1S 5B6
}

Aug. 22, 2007

(C) Copyright 2007, Tao Zheng 


$\begin{array}{ll}\begin{array}{l}\text { Library and } \\ \text { Archives Canada }\end{array} & \begin{array}{l}\text { Bibliothèque et } \\ \text { Archives Canada }\end{array} \\ \begin{array}{l}\text { Published Heritage } \\ \text { Branch }\end{array} & \begin{array}{l}\text { Direction du } \\ \text { Patrimoine de l'édition }\end{array} \\ \begin{array}{l}\text { 395 Wellington Street } \\ \text { Ottawa ON K1A ON4 }\end{array} & \begin{array}{l}\text { 395, rue Wellington } \\ \text { Ottawa ON K1A ON4 } \\ \text { Canada }\end{array}\end{array}$

Your file Votre référence ISBN: 978-0-494-33520-8 Our file Notre référence ISBN: $978-0-494-33520-8$

NOTICE:

The author has granted a nonexclusive license allowing Library and Archives Canada to reproduce, publish, archive, preserve, conserve, communicate to the public by telecommunication or on the Internet, loan, distribute and sell theses worldwide, for commercial or noncommercial purposes, in microform, paper, electronic and/or any other formats.

The author retains copyright ownership and moral rights in this thesis. Neither the thesis nor substantial extracts from it may be printed or otherwise reproduced without the author's permission.
AVIS:

L'auteur a accordé une licence non exclusive permettant à la Bibliothèque et Archives Canada de reproduire, publier, archiver, sauvegarder, conserver, transmettre au public par télécommunication ou par l'Internet, prêter, distribuer et vendre des thèses partout dans le monde, à des fins commerciales ou autres, sur support microforme, papier, électronique et/ou autres formats.

L'auteur conserve la propriété du droit d'auteur et des droits moraux qui protège cette thèse. $\mathrm{Ni}$ la thèse ni des extraits substantiels de celle-ci ne doivent être imprimés ou autrement reproduits sans son autorisation.
In compliance with the Canadian

Privacy Act some supporting forms may have been removed from this thesis.

While these forms may be included in the document page count, their removal does not represent any loss of content from the thesis.
Conformément à la loi canadienne sur la protection de la vie privée, quelques formulaires secondaires ont été enlevés de cette thèse.

Bien que ces formulaires aient inclus dans la pagination, il n'y aura aucun contenu manquant.

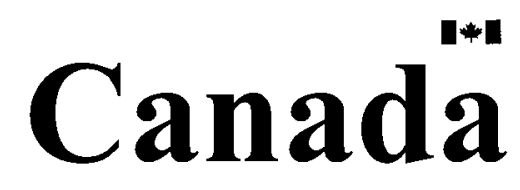




\begin{abstract}
In systems with dynamically changing workloads or components, automated dynamic resource management is promising. It is more effective, responsive and less error prone than static and/or manual resource management. The use of performance models to guide resource management gains the power to explore alternative configurations when making a change. However, it requires building, maintaining and updating a performance model. Queueing network models have been used to analyze static computer systems successfully. This thesis applies a tracking filter technique to update the queueing network model dynamically according to the measurements. The framework proposed in this thesis provides a way to generate the structure of the Layered Queuing Network (LQN) performance model automatically using Application Response Measurement (ARM) standard. The system parameters of the performance model can be measured directly or estimated indirectly using the tracking filters. The extended Kalman filter (EKF) and iterative extended Kalman filter (IEKF) were used to estimate and update system parameters based on the available system measurements with least prediction errors. An in-depth study was made of issues related to the configuring the filter for model parameter confirmation. Given the future system workload predictions, the performance model solver is able to predict the system performance metrics with different configurations in advance to make resource management decisions according to different quality of service (QoS) requirements or policies.
\end{abstract}




\section{ACKNOWLEDGMENTS}

First of all, I would like to express my great thankfulness to my supervisor, Dr. C. M. Woodside for his continuous support, guidance and encouragement over the years. Without his generosity, I would not have completed this research successfully.

I would also like to thank Dr. Marin Litoiu who has been a great mentor and collaborator over the years. My CAS project in IBM Toronto Lab benefited a lot from his work and guidance.

Financial assistance provided by IBM CAS and CITO were greatly appreciated.

Finally, I would like to dedicate this thesis to my dear father Zhihua Zheng, my mother Suzhen Dong for their unlimited love and understanding. 


\section{TABLE OF CONTENTS}

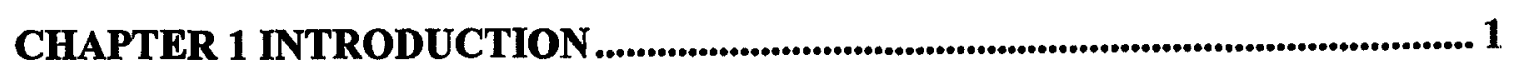

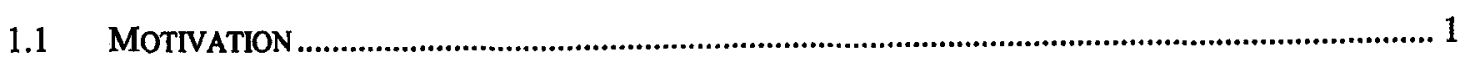

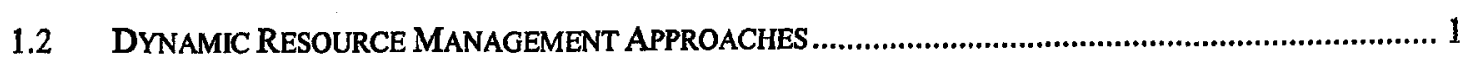

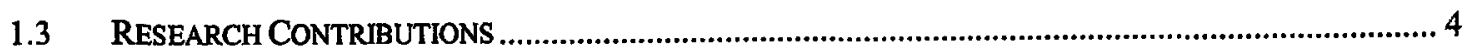

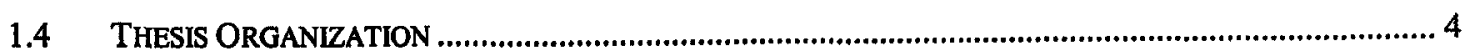

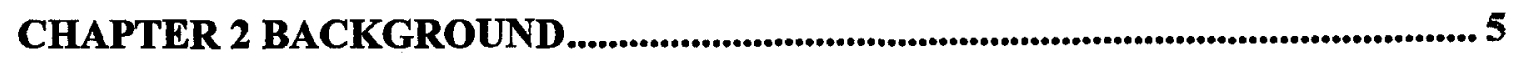

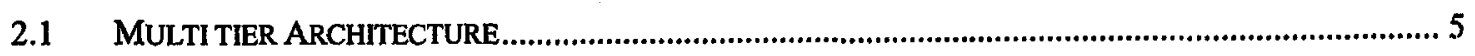

2.2 ADAPTIVE CONTROLS OF COMPUTER AND COMMUNICATION SYSTEMS......................................... 6

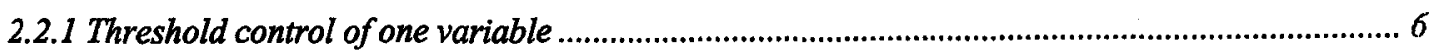

2.2.2 Policy function control for multiple variables....................................................................... 7

2.2.3 Linearized dynamic control ................................................................................................. 8

2.2.4 Queue-based performance model for control ........................................................................... 9

2.2.5 Machine learning approach ................................................................................................ 10

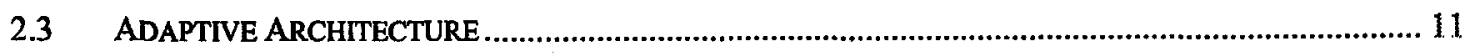

2.4 QUEUEING NETWORK (QN) AND LAYERED QUEUEING NETWORK (LQN) MODEL ...................... 13

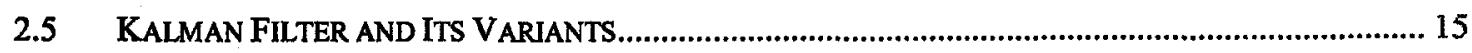

2.5.1 Kalman Filter ............................................................................................................. 15

2.5.2 (Iterated) Extended Kalman Filter ..................................................................................... 16

2.5.3 System Observability ....................................................................................................... 18

CHAPTER 3 PROBLEMS TO SOLVE ............................................................... 19

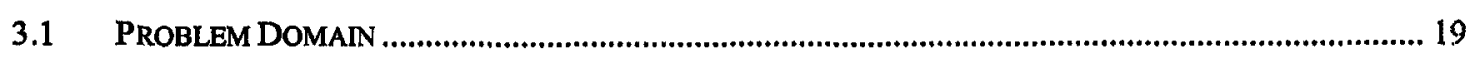

3.2 WEAKNESS OF EXISTING APPROACHES TO RESOURCE MANAGEMENT ......................................... 19

3.3 THE CHOICE OF USING LQN PERFORMANCE MODEL ................................................................... 20

3.4 THE NECESSITY OF TRACKING THE SYSTEM CHANGES ......................................................... 21

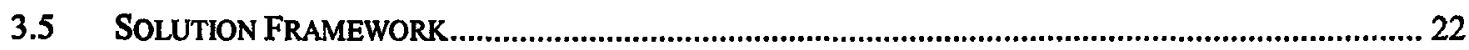

CHAPTER 4 MODEL STRUCTURE EXTRACTOR................................................ 24

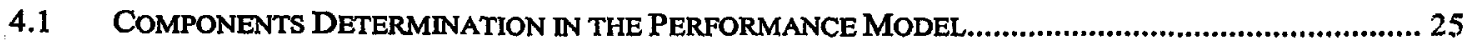

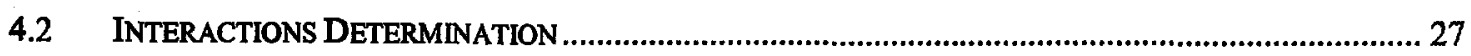

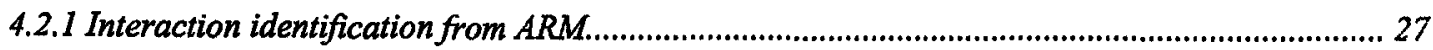

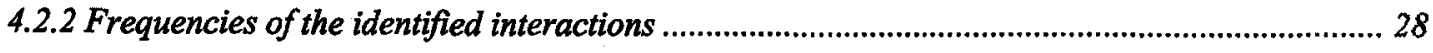

CHAPTER 5 TRACKING FILTER FOR PERFORMANCE MODELS................. 30 


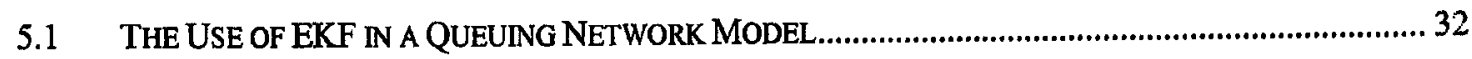

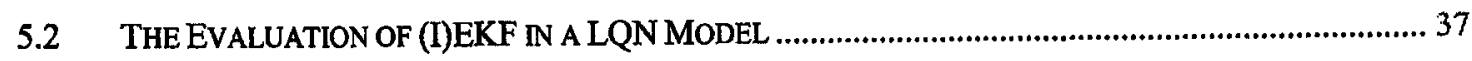

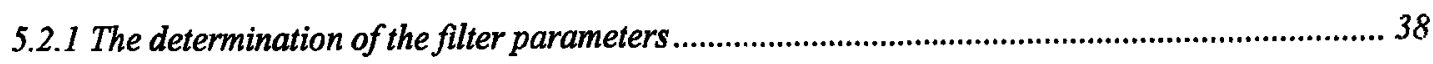

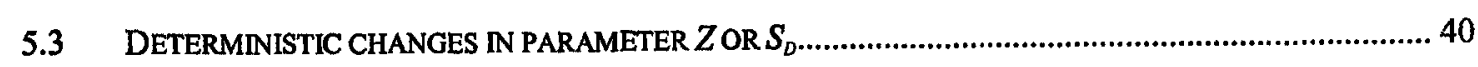

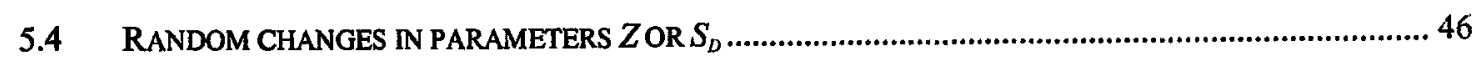

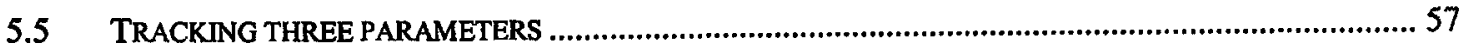

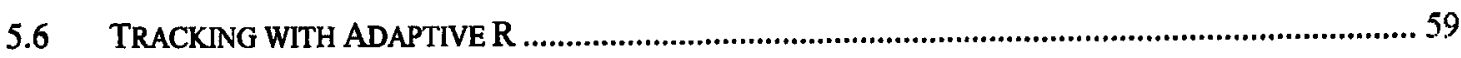

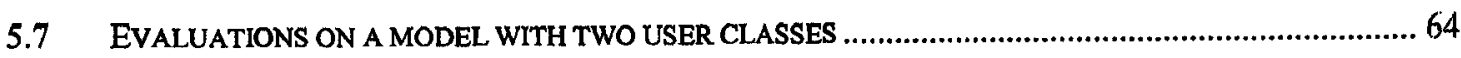

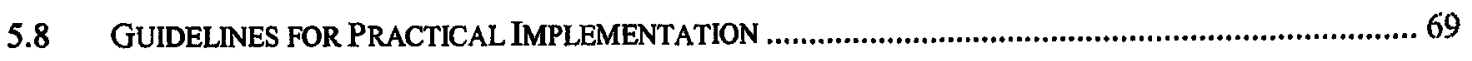

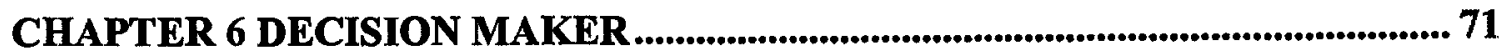

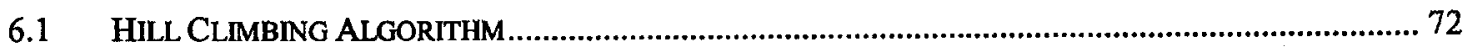

6.2 Provisioning ANd AdMISSION CONTROL IN A Simulated Web SERVER SYSTEM (WSS).......... 74

6.2.1 Simulated Web Server System.................................................................................................. 74

6.2.2 Provisioning Control of the Web Server System ........................................................................ 75

6.2.3 Admission Control of the Web Server System........................................................................... 79

6.3 PROVISIONING AND THREAdS AdJUSTMENT FOR A SIMULATED MULTI TIER SYSTEM WITH Two

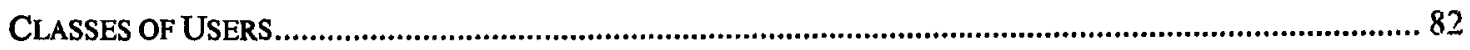

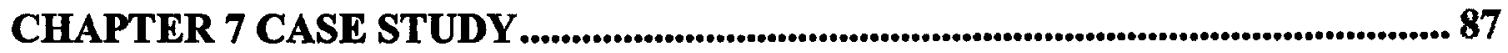

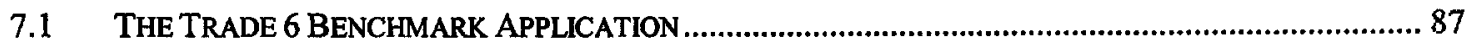

7.2 EVALUATION OF THE PERFORMANCE MODEL ON THE TRADE 6 APPLICATION...............................89

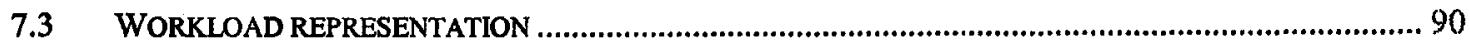

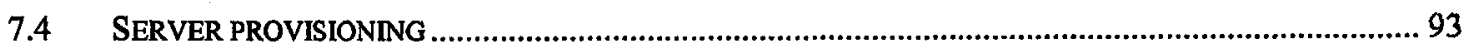

7.4.1 Server provisioning with zero delay .................................................................................... 94

7.4.2 Server provisioning with provisioning delay ............................................................................ 97

CHAPTER 8 CONCLUSIONS................................................................................... 100

REFERENCES...................................................................102

\section{APPENDIX A: OVERVIEW OF CSIMSIMULATOR, CSIMTRACKER AND}

CSIMCONTROLLER.........................................................108

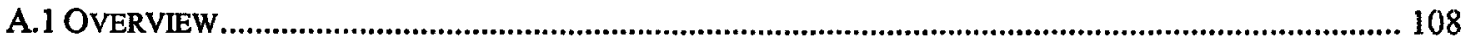

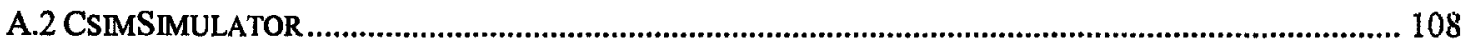

A.3 DATA FORMAT OF THE STATISTIC AND THE COMMUNICATION PROTOCOL BETWEEN CSIMSIMULATOR

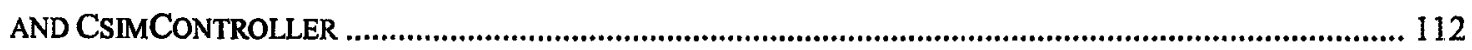


APPENDIX B: LQN MODEL FILES USED IN HT THESIS.......................116

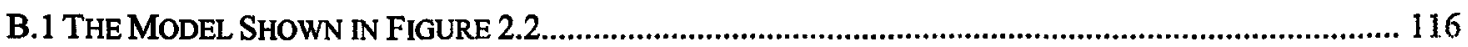

B.2 A MODEL WITH TWO CLASSES OF USERS SHOWN IN FIGURE 5.31 ............................................... 117 


\section{LIST OF FIGURES}

Figure 1.1 Autonomic Manager Control Loop [49] .......................................................................... 2

Figure 1.2 The Architecture of the Framework........................................................................... 3

Figure 2.1 J2EE Architecture [71].......................................................................................... 6

Figure 2.2 The Layerd Queueing Model of a Web Application..................................................... 14

Figure 4.1 The Architecture of the Framework ................................................................................ 24

Figure 4.2 A Common Distributed Application Architecture [38] ................................................... 26

Figure 4.3 An Example of Distributed Transaction [38] ............................................................... 26

Figure 4.4 The Generated Structure of the LQN Model For the Application Shown in Figure 4.2 and

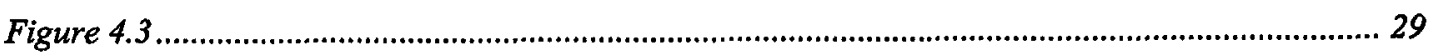

Figure 5.1 The Architecture of the Framework........................................................................... 30

Figure 5.2 A Queueing Model ............................................................................................................... 32

Figure 5.3 A Samplele Transient, with error bars for one standard deviation.................................. 34

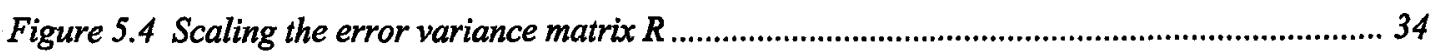

Figure 5.5 Scaling the sampling step time S................................................................................. 35

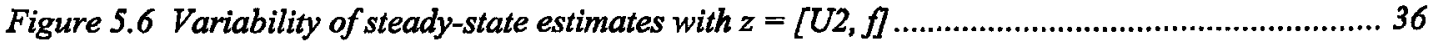

Figure 5.7 Typical transient of the estimates for a two-node model ................................................. 36

Figure 5.8 Standard deviations of estimates using a two-node model ................................................. 37

Figure 5.9 (Case 1) Tracking Performance for a Deterministic Sequence of $Z\left(T=T^{*}\right)(I E K F)$..... 42

Figure 5.10 (Case1) Tracking Performance for a Deterministic Sequence of $Z\left(T=T^{*}\right)(E K F)$....... 43

Figure 5.11 (Case1) Tracking Performance for a Deterministic Sequence of $Z\left(T=10 T^{*}\right)(E K F)$.. 44

Figure 5.12 (Case1) Tracking Performance for a Deterministic Sequence of $Z\left(T=10 T^{*}\right)(I E K F)$.. 44

Figure 5.13 (Case 2) Tracking Performance for a Deterministic Sequence of $S d\left(T=T^{*}\right)(I E K F)$... 45

Figure 5.14 A Trace Fragement of Tracking Random Changes of Z (IEKF)................................... 46

Figure 5.15 (Case 4) MAREs in Tracking Random Changes in User Think Time Z, for Different Lengths of the Measurement Interval (T)

Figure 5.16 (Case 4) fMAREs in Tracking Random Changes in User Think Time Z, for Different Lengths of the Measurement Interval (T) .................................................................................... 52

Figure 5.17 (Case 5) MAREs in Tracking Random Changes Sd, for Different Lengths of the Measurement Interval (T) .................................................................................................... 53

Figure 5.18 (Case 5) fMAREs in Tracking Random Changes in Sd, for Different Lengths of the Measurement Interval (T)

Figure 5.19 (Case 6) MAREs in Tracking Random Changes in User Think Time Z, for Different Values of $C$

Figure 5.20 (Case 6) fMAREs in Tracking Random Changes in User Think Time Z, for Different iv 


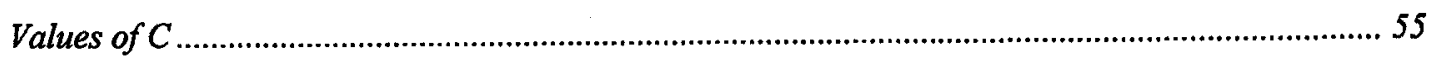

Figure 5.21 (Case 7) MAREs in Tracking Random Changes in Sd, for Different Values of C........... 56

Figure 5.22 (Case 7) fMAREs in Tracking Random Changes in Sd, for Different Values of C.......... 56

Figure 5.23 (Case 8) fMAREs as $T$ is varied for three parameters changing (4 measurements with

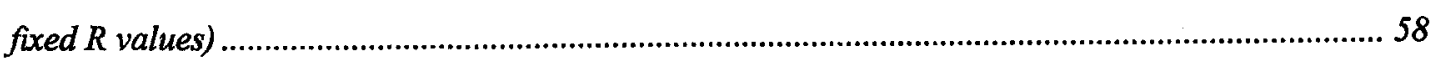

Figure 5.24 (Case 8) fMAREs as $T$ is varied for three parameters changing (7 measurements with

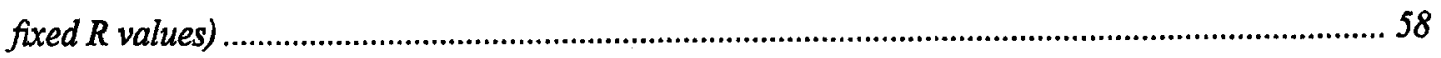

Figure 5.25 (Case 8) fMAREs as $T$ is varied for three parameters changing (4 measurements with adaptive $R$ values from $E q$. (5.13) and Eq. (5.14))

60

Figure 5.26 (Case 8) fMAREs as $T$ is varied for three parameters changing (7 measurements with

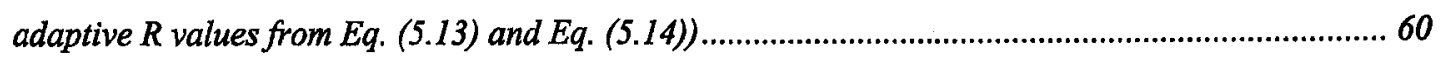

Figure 5.27 (Case 8) fMAREs as $T$ is varied for three parameters changing (4 measurements with

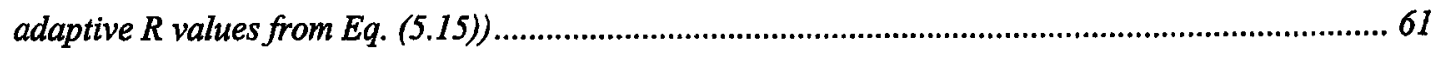

Figure 5.28 (Case 8) fMAREs as $T$ is varied for three parameters changing (7 measurements with adaptive $R$ values from $\mathrm{Eq}$. (5.15)) ...............................................................................................62 62

Figure 5.29 (Case 9) fMAREs as $C$ is varied for three parameters changing (4 measurements) ....... 63

Figure 5.30 (Case 9) fMAREs as $C$ is varied for three parameters changing ( 7 measurements) ....... 63

Figure 5.31 An LQN model with 2 user classes ............................................................................ 64

Figure 5.32 fMAREs as $T$ is varied for the model with 2 user classes ................................................6 66

Figure 5.33 fMAREs as $T$ is varied for the model with 2 user classes ..............................................6 66

Figure 5.34 fMAREs as $T$ is varied for the model with 2 user classes (having entry CPU utilization

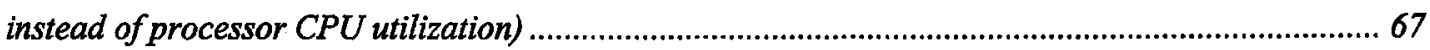

Figure 6.1 The Architecture of the Framework .................................................................................. 71

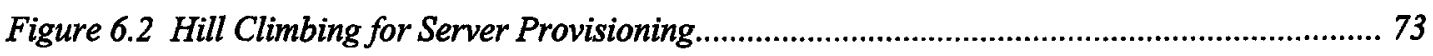

Figure 6.3 Values of Sw and Sd Varied with Time (WSS) ............................................................. 75

Figure 6.4 Flow Chart of Provisioning Control.................................................................................. 78

Figure 6.5 Measured Response Time and Total Servers for Dynamic Provisioning........................... 79

Figure 6.6 Measured Response Time and Number of Users for Admission Control of WSS (SLA_High

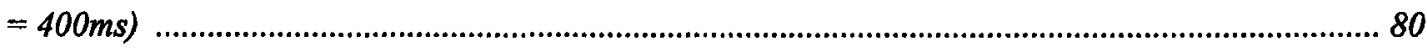

Figure 6.7 Measured Response Time and Number of Users for Admission Control of WSS (SLA_High $=350 \mathrm{~ms})$

Figure 6.8 Values of Class Mix Varied with Time (Multi tier System with 2 Classes of Users).......... 82

Figure 6.9 Measured Response Time and Number of Users of Multi tier System with Provisioning Control Only (SLA1_High $=1 s$ and SLA2_High $=5 s$ ). 83

Figure 6.10 Measured Response Time and Number of Users of Multi tier System with Provisioning Control Only (SLA1_High $=0.9 \mathrm{~s}$ and SLA2_High $=4.5 \mathrm{~s}$ ).

Figure 6.11 Measured Response Time and Number of Users (Multi tier System with Both Provisioning 
Control Only Thread Adjustment)

Figure 7.1 The System Architecture of the Trade 6 Setup ............................................................... 88

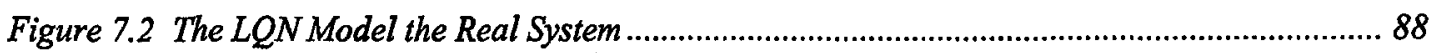

Figure 7.3 Tracking with Varying $N(N$ is Known to the Filter) ..................................................... 90

Figure 7.4 Tracking Results of Trade 6 Application (Tracking both $Z$ and $N$ ) ............................... 91

Figure 7.5 Tracking Results with Fiexed $N^{\prime}\left(N^{\prime}=30\right)$.................................................................... 93

Figure 7.6 Tracking and Forecasting with No Provisioning (zero delay)......................................... 95

Figure 7.7 Tracking, Forecasting and Provisioning (zero delay) ................................................... 96

Figure 7.8 Tracking Result with No Provisioning........................................................................ 98

Figure 7.9 Tracking Results with Reactive Provisioning without Considering Provisioning Delay... 98

Figure 7.10 Tracking Results with Predictive Provisioning control ................................................ 99

vi 


\section{LIST OF TABLES}

Table 4.1 The Mapping between the System and the LQN Model ................................................... 25

Table 4.2 The Component Information for the System Shown in Figure 4.2 and Figure 4.3 .............. 27

Table 5.1 MARE(R) and MARE(Z) for deterministic change of $Z$................................................... 41

Table 5.2 MARE(R) and MARE(Sd) for deterministic change of $S d$................................................ 45

Table 5.3 (Case 3) The MARE(Z), as RFac and QFac are varied using EKF .................................. 48

Table 5.4 (Case 3) The MARE(R), as RFac and QFac are varied using EKF ................................... 48

Table 5.5 (Case 3) The MARE(Z), as RFac and QFac are varied using IEKF ................................. 49

Table 5.6 (Case 3) The MARE(R), as RFac and QFac are varied using IEKF ............................... 49

Table A.1 the programs of the CSIM simulator......................................................................... 109 


\section{LIST OF SYMBOLS AND ACRONYMS}

$a$ : the slope of the linear regression model

$\hat{a}$ : the estimation of $a$

$a^{\prime}:$ the coefficient between two system outputs of a consecutive linearized dynamic model

$\mathbf{A}_{k}$ : the matrix that relates the state vector $\mathbf{x}$ at previous time step $k-1$ to the current step $k$ in the Kalman filter

$b$ : the intercept of the linear regression model

$\hat{b}:$ the estimation of $b$

$b$ ': the coefficient of control input to system output in a linearized dynamic model

$\mathbf{B}_{k}$ : the matrix that relates input vector $\mathbf{u}_{k}$ to the state vector $\mathbf{x}_{k}$

$C$ : the coefficient of variation of a random variable

$C_{i}$ : the coefficient of variation of $x_{i}$

$C_{\text {resource: }}$ the cost of resources in use

$C_{S L A:}$ the cost of violating SLA

$C_{\text {total }}$ : the total cost including $C_{\text {resource }}$ and $C_{S L A}$

D: the service demand vector in the three-node queueing model

$D(i)$ : the service demand of node $i$ in the three-node queueing model

$D_{a}$ : the service demand of the application server in the Trade 6 application

$D_{d b}$ : the service demand of the database server in the Trade 6 application

$e(k)$ : the error between the system output $y(k)$ and reference value $r(k)$ at step $k$

$e_{k+1, i}:$ the measurement error of the $i$ th measurements at step $k+1$ in an adaptive $\mathbf{R}$ solution

$\hat{e}_{k+1, i}:$ the estimated measurement error of the $i$ th measurements in an adaptive $\mathbf{R}$ solution

$f:$ the throughput of requests from users in the three-node queueing model

H: the sensitivity matrix of observations to parameters in the Kalman filter

$\mathbf{H}_{k}$ : the sensitivity matrix of observations to parameters in the Kalman filter at step $k$

$I$ : set of components

I: identity matrix

viii 
$k$ : the step sequence

$K_{P}, K_{I}$ and $K_{D \text { : }}$ the gains of proportional, integral and derivative controller

$\mathbf{K}_{k}$ : Kalman gain in the Kalman filter at step $k$

$M_{d}:$ the number of threads of the database process in the WSS model

$M_{w}:$ the number of threads of the web server process in the WSS model

$N$ : the number of users

$N_{d}$ : the number of replicas of the database server in the WSS model

$N_{w}$ : the number of replicas of the web server in the WSS model

$N_{i}$ : the count that component $i$ is visited during the sampling period

$N_{i j}$ : the count that the identified interaction from component $i$ to component $j$ during the sampling period

O: observability matrix

$\mathbf{P}_{\mathbf{0}}$ : the covariance matrix of the initial estimate

$\mathbf{P}_{k}$ : the covariance matrix of $\hat{\mathbf{x}}_{k}$ at step $k$

$\mathbf{P}_{k}^{-}:$the covariance matrix of $\hat{\mathbf{x}}_{k}^{-}$at $\operatorname{step} k$

Q: covariance matrix of process noise $w$ in the Kalman filter

$\mathbf{Q}_{k}$ : covariance matrix of process noise $\mathbf{w}$ in the Kalman filter at step $k$.

$Q_{i i}:$ the $i$ th diagonal value of $\mathbf{Q}$ matrix

QFac: the factor of the $\mathbf{Q}$ matrix

$r(k)$ : reference value of a control system at step $k$

$\mathbf{R}$ : covariance matrix of measurement noise $\mathbf{v}$ in the Kalman filter

$\mathbf{R}_{k}$ : covariance matrix of measurement noise $\mathbf{v}$ in the Kalman filter at step $k$

$R_{i i}$ : the $i$ th diagonal value of $\mathbf{R}$ matrix

$R F a c$ : the factor of the $\mathbf{R}$ matrix

$R_{a}:$ the response time of the application server in the Trade 6 application

$R_{d b}$ : the response time of the database server in the Trade 6 application

$\hat{R}_{k+1, i, i}$ : the estimation of the $i$ th diagonal value of $\mathbf{R}$ at step $k+1$ in an adaptive $\mathbf{R}$ solution

$R T$ : the mean response time seen by users in the three-node queueing model

$R T(i)$ : the mean response time of node $i$ in the three-node queueing model

$R:$ the user response time 
$S_{d}$ : the service demand of the database server in the WSS model

$S_{w}$ : the service demand of the web server in the WSS model

$S$ : sampling step

SLA_High: the threshold of adding servers in the WSS model

SLA_Low: the threshold of removing servers in the WSS model

SLA1_High: the threshold of adding servers for the user class in the two user classes model

SLA2_High: the threshold of adding servers for the manager class in the two user classes model

SLA1_Low: the threshold of removing servers for the user class in the two user classes model

SLA2_Low: the threshold of removing servers for the manager class in the two user classes model

$T$ : measurement step size

$T^{*}$ : standard measurement step size

$u(k)$ : control input of a linearized dynamic model at step $k$

$\mathbf{u}_{k}$ : a known input vector

$U_{a}$ : the CPU utilization of the application server in the Trade 6 application

$U_{d b}$ : the CPU utilization of the database server in the Trade 6 application

$U_{b}$ : the CPU utilization of the database server in the WSS model

$U_{d}$ : the CPU utilization of the disk in the WSS model

$U_{w}$ : the CPU utilization of the web server in the WSS model

$U(i)$ : the mean utilization of node $i$ in the three-node queueing model

v: the measurement noise

$\mathbf{v}_{k}$ : the measurement noise at step $k$

w: the process noise

$\mathbf{w}_{k}$ : the process noise at step $k$

x: the parameter vector of the Kalman filter

$\mathbf{x}_{k}$ : the parameter vector of the Kalman filter at step $k$

$x_{i}$ : the $i$ th parameter of the parameter vector $\mathbf{x}$

$x_{i, k}$ the mean value of $x_{i}$ at step $k$ 
$\hat{x}_{i, k}:$ the estimation of $x_{i}$ at step $k$

$\hat{\mathbf{x}}_{k}:$ the post-estimation of the state vector $\mathbf{x}$ at step $k$

$\hat{\mathbf{x}}_{k}^{-}$: the pre-estimation of the state vector $\mathbf{x}$ at step $k$

$X_{i:}$ the $i$ th value of $X$ variable in the linear regression model

$\bar{X}$ : the mean value of $X$

$y_{i, k}:$ the prediction of $i$ th measurement at step $k$

$y(k)$ : system output of a linearized dynamic model at step $k$

$Y_{i}$ : the $i$ th value of $Y$ variable in the linear regression model

$\bar{Y}$ : the mean value of $Y$

$Y_{i j}$ : the frequency of the identified interaction from component $i$ to component $j$

$Z$ : the user think time in the WSS model

$\mathbf{z}_{k}$ : the measurement vector at step $k$

$z_{i}$ : the $i$ th measurement of measurement vector $\mathbf{z}$

$z_{i, k}:$ the $i$ th measurement at step $k$

$\alpha$ : an arbitrary value in an adaptive $\mathbf{R}$ solution

$\gamma_{T}$ : the ratio of $T$ to $T^{*}$

$\lambda_{a}$ : the throughput of the application server in the Trade 6 application

$v_{i}$ : the measurement error at sample $i$ in an adaptive $\mathbf{R}$ solution

$\bar{v}$ : the mean value of the measurement error in an adaptive $\mathbf{R}$ solution

API: application programming interface

ARM: application response measurement

CDMA: code division multiple access

CPU: central processing unit

EJB: enterprise Java bean

EKF: extend Kalman filter

fMARE: forward looking mean absolute relative error

HTML: hypertext markup language

IEKF: iterated extended Kalman filter

J2EE: Java ${ }^{\mathrm{TM}} 2$ platform enterprise edition 
JMX: java management extensions

JSP: Java server pages

JVM: Java virtual machine

LQN: layered queueing network

MARE: mean absolute relative error

MDP: Markov decision process

MVA: mean value analysis

QN: queueing network

QoS: quality of service

RPC: remote procedure call

SLA: service level agreement

TPM: Tivoli provisioning manager

UML: unified modeling language

WSS: web server system 


\section{CHAPTER 1 INTRODUCTION}

\subsection{Motivation}

Resources, such as processor, memories and storages, underlie a system's ability to provide adequate quality of service (QoS), such as the response time. Static resource allocation uses a fixed system configuration to face different workloads and scenarios. It is only adequate for systems with small changes. In systems with dynamically changing workloads or components, automated dynamic resource management is a better solution. It is more effective, responsive and less error prone than the static resource allocation. It is able to meet QoS requirements under different workload conditions with limited resources or least costs. For example:

1. An online e-commerce website may have much higher visit rate than usual on holidays, weekends or some special times. The QoS may deteriorate severely if not enough machines are provisioned with high visit rate. However, if enough resources are put to meet the quality of service (QoS) requirement under the worst case conditions, the system is over provisioned most of the time, which wastes resources.

2. In a data center, different services are provided at the same time. If the peak times of those services are quite different, it is possible to shift some of the servers from one to the other to meet QoS all the time with fewer servers.

\subsection{Dynamic Resource Management Approaches}

Dynamic resource management uses basically a feedback control loop to maintain its QoS requirements. The control loop monitors some system measures related to the QoS requirements, then makes changes to the system to meet those QoS requirements. Four approaches were summarized in [29] to deal with the dynamic resource management based on the feedback control loop.

1. Threshold control adds / removes resources according to the thresholds of a measurable parameter. It is the best solution if the system is determined by one parameter only. 
2. Policy function control uses an optimal solution to deal with systems with more than one parameter. It is an extension of approach 1.

3. Linearized dynamic control uses control theory to build a controller to achieve desired QoS on a linearized system model. It works efficiently on linear systems, nearlinear systems or nonlinear systems operating within a linear range.

4. Queuing network performance model has been used to analyze some types of nonlinear system (e.g. e-commerce, telecommunication systems). However, it is too difficult or too expensive to measure some of the model parameters (e.g. service times of the queuing nodes) in real systems. The use of tracking filter proposed in [60][65] provides a way to estimate the hidden model parameters from the available measurements dynamically. It makes this approach more practical to solve the resource management problems in dynamic systems.

Recently, machine learning provides a new approach for dynamic resource management. It uses trial-and-error methodology to derive best configuration for the specific system state. The best configuration and system state pair are remembered to guide the future system control.

IBM proposed autonomic computing [49] architecture as a generic solution of automated dynamic resource management. Autonomic computing is a self-managing environment and has the ability of self-configuring, self-healing, self-optimizing, and self-protecting. The managed resources are controlled by an autonomic manager with a control loop embedded (Figure 1.1) via the sensor and effector. The control loop in the autonomic manager includes the following parts: monitor, analyze, plan, execute and knowledge.

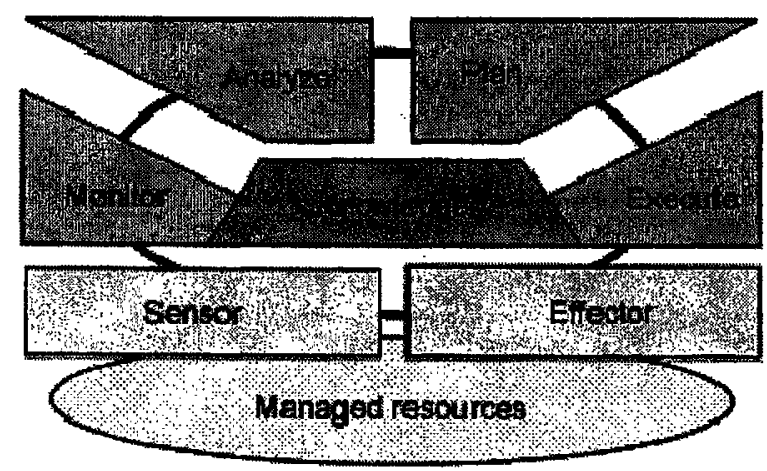

Figure 1.1 Autonomic Manager Control Loop [49] 
All the dynamic resource management approaches mentioned before could be fitted to this architecture.

This thesis uses an extended queueing network performance model called layered queueing network (LQN)[14][15][44][58][59] and a tracking filter to deal with the dynamic resource managements. It proposes a framework for automated dynamic resource managements based on the IBM's autonomic computing architecture (Figure 1.2). In this framework, a performance model will be built automatically from the system design diagrams (e.g. UML diagrams) and measurements. The performance model building in this framework has 2 parts, to extract the model structure and to estimate the model parameters. The structure of the performance model can be generated by Model Structure Extractor from the system design diagrams. The parameters of the performance model are monitored directly by the System Monitor or estimated and updated indirectly by the Tracking Filter. Based on the analysis results, the Decision Maker provides a new optimized system reconfiguration according to meet QoS requirements using optimization methods, heuristic strategies or policies.

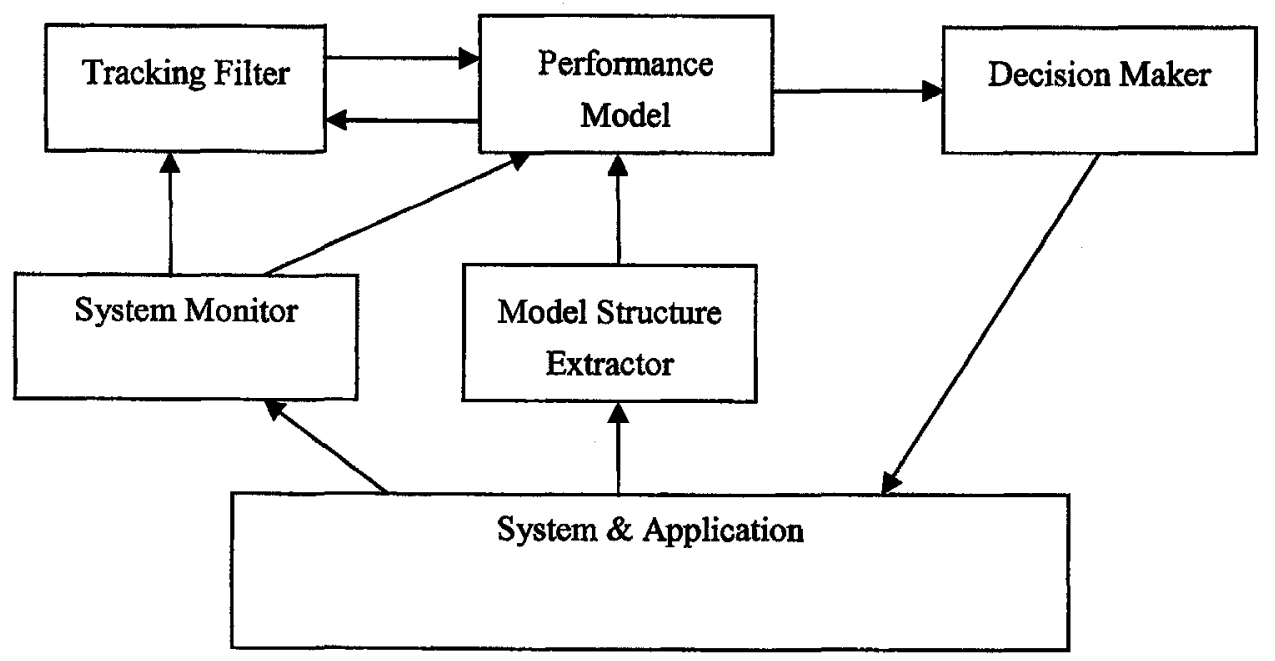

Figure 1.2 The Architecture of the Framework 


\subsection{Research Contributions}

The followings are the contributions of this research:

1. Apply the tracking filters to estimate and update LQN performance models for the first time. It makes it more practical to use the queuing network based performance models for dynamic resource management in autonomic computing systems. This research is the first to apply the Kalman filter for performance model tracking.

2. Evaluate and improve the tracking quality using extended Kalman filter (EKF) and iterative extended Kalman filter (IEKF), with different parameters setting on both real systems and simulated systems. The evaluations suggest good settings (e.g. the set of measurements, covariance matrix of process errors $\mathbf{Q}$, covariance matrix of measurement errors $\mathbf{R}$, measurement step size etc.) to make the tracking filter work best for real and complicated systems.

3. Implementation of a simulated tracking and control test-bed. The simulator is generated automatically from an LQN model. The controller uses a hill-climbing algorithm to optimize the system configuration based on the parameter estimation from tracking filters.

\subsection{Thesis Organization}

This thesis is organized as follows. Chapter 2 gives the background on the adaptive control, tracking filter and related topics. Chapter 3 describes the problems to be solved in this research. Chapter 4 provides a method to generate the structure of performance model from ARM standard and system architecture diagrams and UML diagrams. Chapter 5 investigates and evaluates the tracking filters in the LQN performance models. Chapter 6 discusses the strategies used for decision making in the framework. The strategies were applied to simulated systems and showed the effectiveness. Chapter 7 provides case studies on a real benchmark application. Chapter 8 gives the conclusions finally. 


\section{CHAPTER 2 BACKGROUND}

The world is always changing. By using new technologies, the computer hardware cost has fallen dramatically for many years while the performance increases significantly. At the same time, software systems become more and more complicated, which increases management cost (including the resource management cost). In systems with dynamic changing workloads or components, automated dynamic resource management is a promising and efficient solution. It is more effective, responsive and less error prone than the static and/or manual resource management to meet QoS requirements under different workload conditions with limited resources or least cost.

\subsection{Multi tier Architecture}

In software engineering, multi tier architecture (usually refers to three tier architecture) is an extension of traditional two tier client-server architecture. A typical three tier architecture consists of the presentation tier, business logic tier and data tier. Any of the three tiers can be upgraded independently when the requirements or system change. The multi tier architecture improves the scalability, reusability, data integrity and security. In the thesis, a system that uses the multi tier architecture is called a multi tier system. The target system of this thesis is the multi tier information system.

The Java ${ }^{\mathrm{TM}} 2$ Platform, Enterprise Edition (J2EE) architecture is an example of multi tier architecture. It is "the industry standard for developing portable, robust, scalable and secure server-side Java applications" [70]. It provides a solution to develop multi tier enterprise services faster and more efficient.

Figure 2.1 shows an example of J2EE architecture. A J2EE application system typically includes the following tiers:

- Client tier: The client tier usually consists of Java applications, applets or HTMLs. This is the tier which users interact with directly.

- Middle tier: The middle tier consists of 2 parts: the presentation part and business logic part. The presentation part includes the web components, such as the Servlets 
and JavaServer Pages (JSPs) which communicate with client tier. The business logic part includes the enterprise Java beans (EJB) and Web Services which encapsulate reusable, distributable business logic for the application.

The middle tier is usually an application server. The best known commercial J2EE application servers are WebSphere (IBM), WebLogic Server (BEA) and JBoss (Red Hat).

- Date tier: The data tier stored the enterprise's data in a relational database typically.

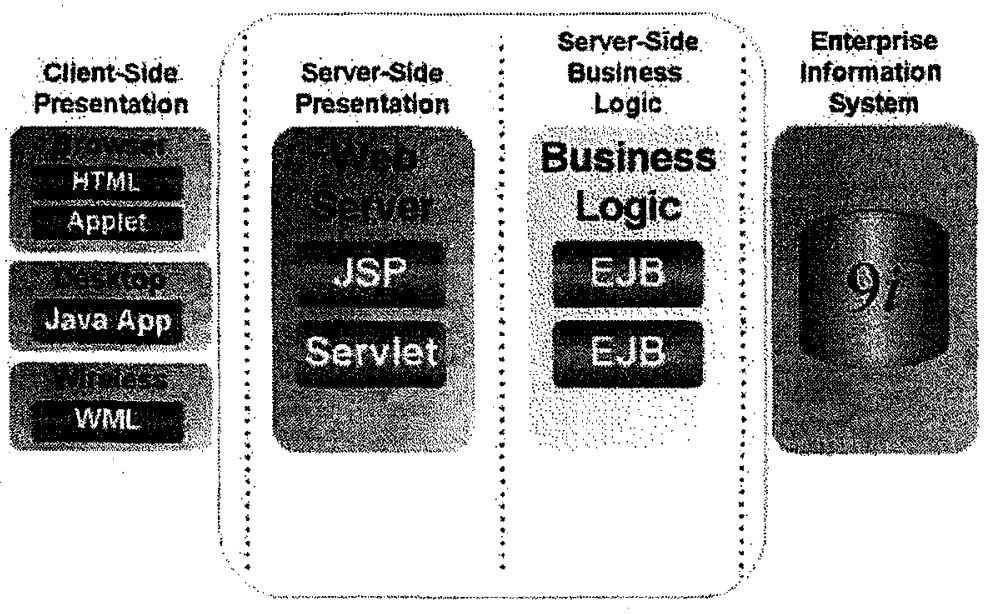

Figure 2.1 J2EE Architecture [71]

\subsection{Adaptive Controls of Computer and Communication}

\section{Systems}

The feedback control loop has been used to manage system resources for a long time. Four types of adaptive controls are summarized in [29]: threshold control, policy function control, linearlized dynamic control and queue-based performance model control. New types of controls appear in recent researches as well.

\subsubsection{Threshold control of one variable}


The controller makes decisions according to the value of a single control variable. If the control variable reaches a certain threshold, the controller will change the system to work at a related state or level. Threshold control is widely used for admission control and congestion control in computing and communication systems. In web servers, the admission control may be based on the mean response time. The request is accepted only if the mean response time is under a threshold. In [45], this type of admission control is extended to multiple classes of users with different priorities. For shared web servers, only the requests with high priorities will be accepted when the mean response time is within a range. For example if a web server is shared by 2 classes of users, 2 thresholds, $R_{d i f f}$ and $R_{\max }$ are set for admission control. All the requests will be accepted when the mean response time is less than the threshold $R_{\text {diff }}$ and all the requests will be rejected if the mean response time is more than the threshold $R_{\max }$. When the mean response time is between $R_{d i f f}$ and $R_{\max }$, the requests from low priority class will be rejected. In communication systems, the connection/call admission control may make decisions according to the threshold of the number of flows in the system, mean service time of a connection or total received power in CDMA systems [40] to meet QoS requirements.

The threshold control reduces the frequency of control actions when the system is in a desirable regime. The cost of control is reduced to minimum. Threshold control is the simplest and most efficient control comparing with other adaptive controls.

The threshold control assumes that only one parameter affects the system behavior. In many systems, a number of parameters may affect the system behavior simultaneously. Threshold control is the best choice for "simple" systems only.

\subsubsection{Policy function control for multiple variables}

Threshold control can be generalized to multiple variables and control levels using an optimal policy. The policy can be determined using different optimization strategies.

\section{Markov Decision Process Model}

A Markov decision process (MDP) [40] model has a set of states $S$ and actions $A$. The model has transition probabilities $P_{i j}$ from state $s_{i}$ to state $s_{j}$. A reward (or cost) $R(s, a)$ is assigned to an action $a$ taken in a state $s$. A policy is a rule to define an action $a$ from 
state $s$. An optimal policy can be calculated with the maximum reward (or minimum cost) in steady states. In [1], a Markovian model has been used to compute optimal policy for a mobile multi-media application which could play video clips, movies etc. from a mobile device. For different number of mobile devices users and QoS requirements, an optimal policy with three decision parameters will be selected for the least cost. In [13] [46] the Markov decision models are used to reconfigure the distributed systems to cope with component failures, repairs and workload changes based on the optimization of the average reward per time unit.

The Markov decision process model is a promising approach if the number of states in the model is limited. It may suffer from state space explosion problem for systems with large number of states. There are ideas for dealing with the state space explosion problem using cluster of states.

A recent research studied a policy-based management of an E-commerce business simulation [25]. All the controls were guided by a number of predefined policies. However, it is very difficult to derive good policies dynamically.

\subsubsection{Linearized dynamic control}

Hellerstein and his co-workers [18] and others have used linearized dynamic models to model a computer system by equations as

$$
y(k+1)=a^{\prime} y(k)+b^{\prime} u(k)
$$

where $y(k)$ and $u(k)$ are the outputs and inputs of the target system at time $k$. The inputs are some control quantities such as the buffer size and the maximum number of users in a system. The outputs are some performance measures such as the CPU utilizations.

The coefficients $a^{\prime}$ and $b^{\prime}$ can be estimated by statistical methods such as least square regression. They designed proportional-integral-derivative (PID) controllers to control the QoS towards a desired value based on a well-developed control theory:

$$
\begin{aligned}
& u(k)=K_{p} e(k)+\left(u(0)+K_{I} \Sigma_{j=1 \ldots k} e(j)\right)+K_{D}(e(k)-e(k-1)) \\
& e(k)=y(k)-r(k)
\end{aligned}
$$

where $K_{P}, K_{I}$ and $K_{D}$ are the gain parameters of the PID controllers, $r(k)$ is the reference value of the target system at time $k$ and $e(k)$ is the error between the output of 
the target system and the reference value at time $k$.

The gain parameters of the controllers (i.e. $K_{P}, K_{I}$ and $K_{D}$ ) can be determined by pole placement or root locus methods. The reference of the controller is carefully chosen so that the target system is approximately a linear system around this operating point. Even if the real system might be nonlinear, the equation 2.1 may still hold around the selected reference values approximately.

Various example systems have been successfully controlled. Hellerstein et al. controlled the KeepAlive parameter, the maximum time the thread in wait state, of the Apache server [8] and the maximum number of users (MaxUsers) parameter in Lotus Notes mail server [17] to achieve required QoS. Linearized dynamic control has been aused by Abdelzaher et al. [2][3] for overload protection and service differentiation as well. They used a linear equation to calculate the CPU utilization. The CPU utilization was controlled to be just below a threshold that in turn guarantees the QoS requirement by providing different levels of degraded contents for different classes of users under overload conditions. In [32], relative delay guarantees are maintained by resource allocation with an additional queuing predictor.

A control-theoretic approach to flow control was applied to network communication area [26]. The communication channels adapted their data transfer rate to changes in network state with best effort.

The theory of control based on time-varying models was studied intensively by Åström and his co-workers [5], and was applied to time-varying linear systems and to nonlinear systems in which the operating point changed. They designed self-tuning regulator to update the linearized system model dynamically.

\subsubsection{Queue-based performance model for control}

Queue-based performance models, including queueing network (QN) model, layered queueing network (LQN) model and their variants, have been used to analyze the static performance features of the computer and communication systems successfully. Recently, those performance models are applied to predict the performance metrics of nonlinear dynamic systems and reconfigure the systems combining the use of optimization strategies. Menasce used Markov chain and queueing network model to 
represent a multithreaded server computer system [33]. A QoS controller determines the best configuration of the system by using searching algorithms (e.g. hill-climbing, beam search algorithm) according to a QoS metric based on the user response time, throughput and probability of rejection. Litoiu used layered queueing model to predict the performance metrics of a multi class multi tier distributed systems [28]. By using linear and nonlinear programming techniques, an object allocation was presented to optimize the performance metric.

The queue-based performance model has the ability to predict performance metrics for types of nonlinear systems. It was widely used to analyze the static computer and communication systems. However, it has not been widely used in dynamic systems because of the difficulties to get model parameters (e.g. service demands of the nodes) in real systems. Some recent researches used queueing models to estimate the model parameters using measurements that are easy to get. Liu et al. inferred the service demands of queueing models by minimizing the estimation errors of the end-to-end response times [31]. Urgaonkar et al. presented a queueing network model for multi tier internet services and applications [54]. The model was validated and applied to dynamic capacity provisioning in a data center. Zhang $e t$ al. used regression-based approximation to estimate the service demands of different transactions, then used queueing network model to predict performance metric with different transaction mixes [62].

\subsubsection{Machine learning approach}

Recently, machine learning becomes a new approach for dynamic resource management. It uses trial-and-error methodology to derive best configuration for the specific system state. The best configuration and system state pair are remembered to guide the future system control.

In [55][50], reinforcement learning [50] were applied to learn the optimal management policy automatically. The optimal policy could be derived from Markov Decision Process (MDP). In [8], another machine learning algorithm called K-nearest-neighbours was used for database provisioning.

Machine learning is a knowledge free approach. However, the learning phase may take 
a long time for large systems. If the system varies too much, the previous exploration might not be able to provide good configuration for current or future system states. Furthermore, some of the machine learning algorithm (e.g. MDP) may suffer state explosion problem for large systems.

In [52], a hybrid approach which combines the queueing model and machine learning was proposed. The performance model was used to deal with resource management first. At the same time, machine learning was started. When the learning phase was done, the performance model was switched to the machine learning.

\subsection{Adaptive Architecture}

Some IT companies have proposed blueprints for dynamic resource management. IBM proposed the autonomic computing architecture as a generic solution of resource management problems in dynamic systems. Autonomic computing is "a computing environment with the ability to manage itself and dynamically adapt to change in accordance with business policies and objectives" [19]. It is a self-managing environment and has the ability of self-configuring, self-healing, self-optimizing, and self-protecting.

- Self-configuring: Reconfigure the systems to adapt to changing environment dynamically.

- Self-healing: detect, diagnose system failures and recover the system automatically.

- Self-optimizing: monitor and tune the system automatically.

- Self-protecting: detect and identify malicious behaviors to protect the system.

The autonomic computing systems consist of managed resources, the touch points and autonomic managers. Autonomic managers are the core components in the autonomic computing systems. They manage other software or hardware components using a control loop. It makes the autonomic computing systems different from normal systems

The control loop in the autonomic managers (Figure 1.2) has 5 parts:

- Monitor: collects available system information from the managed resources via the sensors

- Analyze: analyzes the system information to determine if changes are needed 
according to the policies and service level agreements.

- Plan: creates to a change plan of the managed resources

- Execute: performs the necessary changes to the system via the effectors

Those 4 parts mentioned above constitute a control loop

- Knowledge: maintains the system information data shared by the other 4 parts.

IBM has a series of products which implement this architecture.

In addition to the IBM's autonomic computing architecture, HP proposed the Adaptive Enterprise Architecture for the enterprise solutions. It provides strategies for building adaptive IT environments that are able to improve the performance, minimize the costs and risks even when the business needs will grow and change.

Microsoft has Dynamic Systems Initiative to help IT organizations to build selfmanaging dynamic systems that can increase productivity and responsiveness to changing business demands, reduce the development and maintenance costs.

All those companies have similar objectives to deal with the complexities in dynamic systems. IBM and HP focus on the resource management of dynamic systems (e.g. data centers). Microsoft pays more attentions to the life cycles of the software products to decrease the complexity. To our knowledge, up to now, HP and Microsoft provides only the structure of the architecture and principles of designing self-managing dynamic system while IBM provides more details of designing systems dealing with dynamic resource managements.

The framework proposed in the thesis is based on the IBM's autonomic computing architecture. It is designed to build self-configuring and self-optimization autonomic managers for automated dynamic resource management. IBM has provided products and tools to cover the Execute part in the autonomic manager. The thesis is more interested in the other parts of the control loop in the autonomic managers. The systems discussed in the research are assumed to be able to perform the changes. The Execute part will be omitted in the framework. 


\subsection{Queueing Network (QN) and Layered Queueing Network (LQN) Model}

Queueing Network (QN) Model is a performance model used widely in computer systems and communication systems. A special type of closed QN model with some restrictions called closed product-form queueing network model can have computationally efficient solutions such as Mean Value Analysis [43].

The layered queueing network (LQN) model, presented by Woodside et al., is a performance model for systems with distributed software servers [14][15][44][58][59]. It extends the queueing network model and can be used to model layered and distributed systems with a variety of different system behavior (e.g. hardware devices, software processes, nested services, precedence constraints and multithreads). It has been applied to both enterprise system and embedded systems.

Figure 2.2 is an example of the LQN model of a web application.

In a LQN model, tasks (the squares with bold lines in Figure 2.2) represent software objects that may execute concurrently. A task may have a number of threads running simultaneously on a specific processor (the ovals in Figure 2.2). A task may have some entries (the squares with light lines to the left of the tasks) that provide different services. In queueing terms, a task is a server and each entry is a class of service. An entry consists of some "activities" or "phases" which are the smallest execution units. The activities may have complex precedence relationships (e.g. AND fork, join, OR fork, join) with each other [16].

Three types of communications are supported in LQN model: synchronous call, asynchronous call, and forwarding call:

Synchronous call: sender blocks when waiting for a reply from receiver. This is the pattern of standard Remote Procedure Call (RPC). All the calls in Figure 2.2 are synchronous calls.

Asynchronous call: sender does not block after sending a request. No replies are required. Only request messages exist in asynchronous call.

Forwarding call: The receiver might forward a synchronous call to the third task instead of replying it directly. The third task might reply to it or forward it further. The 
receiver does not block after forwarding. The final receiver will send a reply to the blocked sender directly.

Both analytic and simulation tools are supported for LQN models. The input format of LQN model can be found in [16].

LQN is a suitable tool to model the multi tier information system such as J2EE application systems. The layered feature represents the multi tier system very well. As the EJBs are the software components, they could be modeled as tasks or entries.

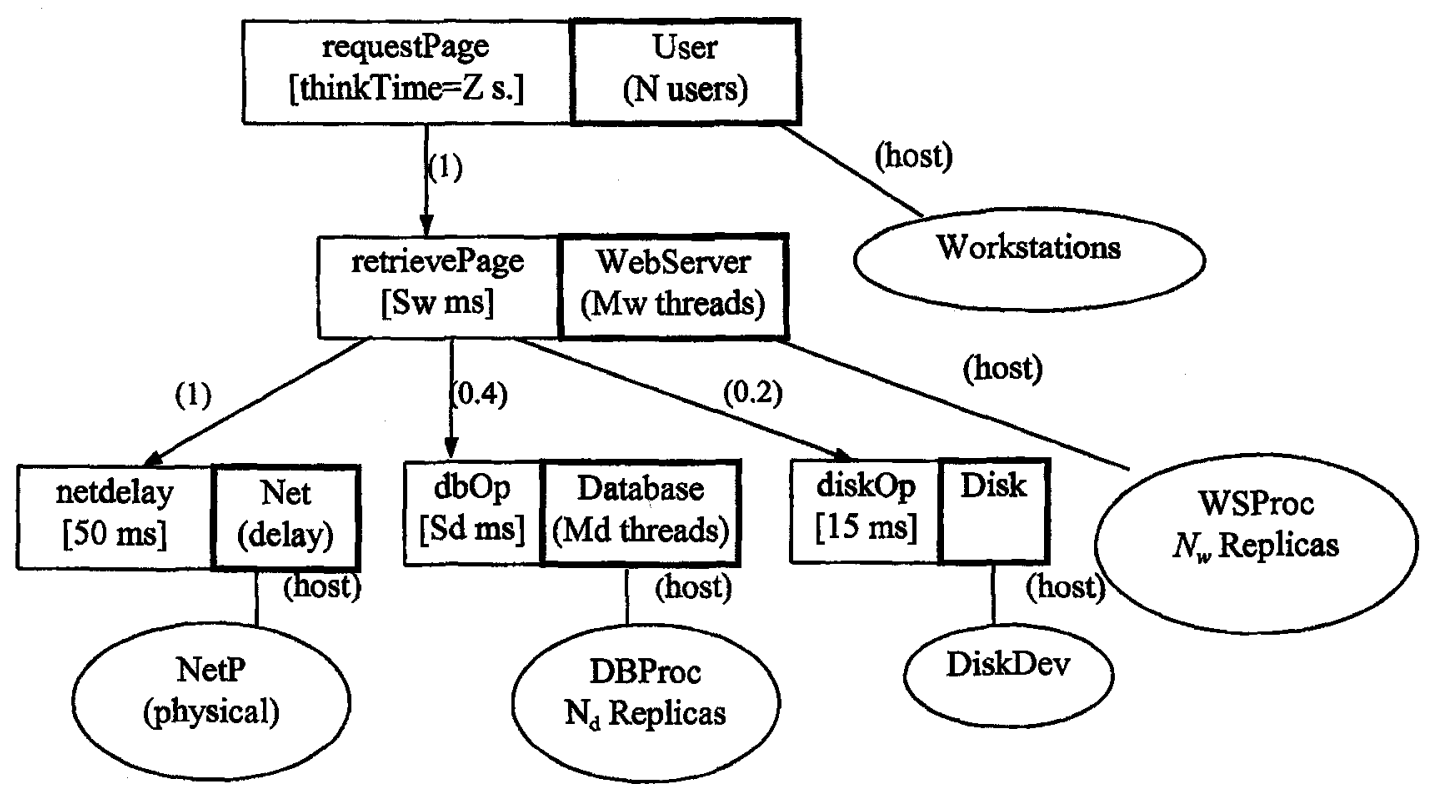

Figure 2.2 The Layered Queuing Model of a Web Application

In Figure 2.2, the web application has $N$ users with think time $Z$ seconds, which cycles between thinking and sending a request to the WebServer with $M_{w}$ threads. The user request requires a CPU demand of $S_{w} \mathrm{~ms}$ on the WebServer, one network latency of 50 $\mathrm{ms}$, and on average 0.4 database operations. Each database operation requires a CPU demand of $S_{d} \mathrm{~ms}$, and 0.2 disk operations with a default $15 \mathrm{~ms}$ CPU demand per visit. The Database is multithreaded task with $M_{d}$ threads. The WebServer and Database tasks are hosted on WSProc and DBProc processors. The processors belong to clusters with $N_{w}$ and 
$N_{d}$ replicas respectively.

In the thesis, the multi tier system it co-located, that is all the physical machines are located closely that the network is ignored. If the physical machines are located remotely, the network delay can be modeled as a simple fixed latency.

\subsection{Kalman Filter and Its Variants}

\subsubsection{Kalman Filter}

The Kalman filter [24] was proposed by R. E. Kalman in 1960. It described a recursive solution to the discrete data linear filtering problem.

For linear discrete system, the model of the state vector $\mathbf{X}$ at step $k$ is

$$
\mathbf{x}_{k}=\mathbf{A}_{k} \mathbf{x}_{k-1}+\mathbf{B}_{k} \mathbf{u}_{k}+\mathbf{w}_{k}
$$

with a measurement vector $\mathbf{z}$

$$
\mathbf{z}_{k}=\mathbf{H}_{k} \mathbf{x}_{k}+\mathbf{v}_{k}
$$

where

$\mathbf{A}_{k}$ is the matrix that relates the state vector at previous time step $k-1$ to the current step $k$

$\mathbf{u}_{k}$ is a known input vector

$\mathbf{B}_{k}$ is matrix that relates the state vector $\mathbf{x}_{k}$, and input vector $\mathbf{u}_{k}$

$\mathbf{w}_{k}$ is the process noise with zero mean and covariance matrix $\mathbf{Q}$

$\mathbf{v}_{k}$ is the measurement noise with zero mean and covariance matrix $\mathbf{R}$

$\mathbf{z}_{k}$ is the measured value for $\mathbf{z}$ at step $k$.

$\mathbf{H}_{k}$ is the sensitivity of observations to parameters

Equation (2.4) is called the process model of the linear system and equation (2.5) is referred as the measurement model as well.

The optimal estimate $\hat{\mathbf{x}}$ at time $k$ minimizes the sum of squared errors $\left(\hat{\mathbf{x}}-\mathbf{x}_{k}\right)$ of the states, given the sequence of observations $\mathbf{z}_{l}, \ldots \mathbf{z}_{k}$, and an initial estimate $\hat{\mathbf{x}}_{0}$ (which is normally distributed with initial covariance matrix $\mathbf{P}_{0}$ ). The optimal filter executes the following operations at step $k$ (see, e.g. [19][24]): 
1. project the state $\hat{\mathbf{x}}_{k}^{-}$ahead:

$$
\hat{\mathbf{x}}_{k}^{-}=\mathbf{A}_{k} \hat{\mathbf{x}}_{k-1}+\mathbf{B}_{k} \mathbf{u}_{k}
$$

2. project the estimated covariance matrix $\mathbf{P}^{-}$, for the estimates of $\mathbf{x}$ :

$$
\mathbf{P}_{k}^{-}=\mathbf{A}_{k} \mathbf{P}_{k-1} \mathbf{A}_{k}^{\mathrm{T}}+\mathbf{Q}_{k}
$$

3. compute the Kalman gain $\mathbf{K}_{k}$ as:

$$
\mathbf{K}_{k}=\mathbf{P}_{k}^{-} \mathbf{H}_{k}^{\mathbf{T}}\left(\mathbf{H}_{k} \mathbf{P}_{k}^{-} \mathbf{H}_{k}^{\mathbf{T}}+\mathbf{R}_{k}\right)^{-1}
$$

4. make the measurement $\mathbf{z}_{k}$ and correct the state vector:

$$
\hat{\mathbf{x}}_{k}=\hat{\mathbf{x}}_{k}^{-}+\mathbf{K}_{k}\left(\mathbf{z}_{k}-\mathbf{H}_{k} \hat{\mathbf{x}}_{k}^{-}\right)
$$

5. correct the error covariance $\mathbf{P}_{k}$ :

$$
\mathbf{P}_{k}=\left(\mathbf{I}-\mathbf{K}_{k} \mathbf{H}_{k}\right) \mathbf{P}_{k}^{-}
$$

with the additional notation:

$\hat{\mathbf{x}}_{k}^{-}$is the pre-estimation of the state vector $\mathbf{X}$ at step $k$

$\hat{\mathbf{x}}_{k}$ is the post-estimation of the state vector $\mathbf{x}$ at step $k$

$\mathbf{P}_{k}^{-}$is the covariance matrix of $\hat{\mathbf{x}}_{k}^{-}$at step $k$

$\mathbf{P}_{k}$ is the covariance matrix of $\hat{\mathbf{x}}_{k}$ at step $k$

$\mathbf{R}_{k}$ is measurement error covariance matrix at step $k$.

$\mathbf{Q}_{k}$ is disturbance error covariance matrix at step $k$.

The Kalman filter itself has an feedback loop embedded. The state vector $\mathbf{x}$ is first estimated from the process model (Eq. (2.4)). Then the estimation is corrected according to the difference between the measurements and predictions (Eq. (2.9)).

\subsection{2 (Iterated) Extended Kalman Filter}

For nonlinear system, the predictions based on $\mathbf{x}$ are no longer linear equations. The process model and measurement model are modified as follows:

$$
\begin{aligned}
& \mathbf{x}_{k}=\mathbf{f}\left(\mathbf{x}_{k-1}, \mathbf{u}_{k}\right)+\mathbf{w}_{k} \\
& \mathbf{z}_{k}=\mathbf{h}\left(\mathbf{x}_{k-1}\right)+\mathbf{v}_{k}
\end{aligned}
$$

The extended Kalman filter (EKF) [19] linearizes the process model $\mathbf{h}\left(\mathbf{x}_{k}\right)$ and measurement model around the state estimate for nonlinear system. In this thesis, we 
assume $\mathbf{u}_{k}=0, \mathbf{Q}_{k}$ and $\mathbf{R}_{k}$ do not vary with time and $\mathbf{A}_{k}=\mathbf{I}$, the identity matrix for simplicity.

The EKF equations work as follows:

1. project the state ahead with $\mathbf{w}_{k}=0$ :

$$
\hat{\mathbf{x}}_{k}^{-}=\hat{\mathbf{x}}_{k-1}
$$

2. project $\mathbf{P}_{k+1}$, the estimated covariance matrix for the estimates of $\mathbf{x}$ :

$$
\mathbf{P}_{k}^{-}=\mathbf{P}_{k-1}+\mathbf{Q}_{k}
$$

3a compute the linearization of the output function $\mathbf{H}_{k}$ at $\hat{\mathbf{x}}_{k}^{-}$,

where $\mathbf{H}_{k}=\partial \mathbf{h} / \partial \mathbf{x}$ is the linear term in the Taylor expansion of $\mathbf{h}(\mathbf{x})$.

$\mathbf{H}_{k}$ can be calculated numerically by increasing $\hat{\mathbf{x}}_{k}^{-}$a small percentage (e.g. $1 \%$ ).

$3 \mathrm{~b}$ compute the Kalman gain $\mathbf{K}_{k}$ :

$$
\mathbf{K}_{k}=\mathbf{P}_{k}^{-} \mathbf{H}_{k}{ }^{\mathbf{T}}\left(\mathbf{H}_{k} \mathbf{P}_{k}^{-} \mathbf{H}_{k}{ }^{\mathbf{T}}+\mathbf{R}_{k}\right)^{-1}
$$

4. correct the state vector using the error of the nonlinear predicted output:

$$
\hat{\mathbf{x}}_{k}=\hat{\mathbf{x}}_{k}^{-}+\mathbf{K}_{k}\left(\mathbf{z}_{k}-\mathbf{h}\left(\hat{\mathbf{x}}_{k}^{-}\right)\right)
$$

5. correct the error covariance $\mathbf{P}_{k}$ :

$$
\mathbf{P}_{k}=\left(\mathbf{I}-\mathbf{K}_{k} \mathbf{H}_{k}\right) \mathbf{P}^{-}{ }_{k}
$$

A variant of EKF called iterative extended Kalman filter (IEKF) [19] linearizes the process and measurement model around the updated state estimate instead of the previous state estimate in EKF. IEKF is achieved by iterations using the previous state estimate as an input to generate a new state estimate. The iteration will continue until the 2 successive state estimates are close enough.

The EKF and its variants are used to estimate positions in robotics [46], locations of aircraft from radar observations (and in navigation generally)[6]. When $\mathbf{f}(\mathbf{x})$ or $\mathbf{h}(\mathbf{x})$ are nearly linear, the filter is expected to have near-optimal properties. The optimality and convergenice properties depend on the functions $\mathbf{f}(\mathbf{x})$ or $\mathbf{h}(\mathbf{x})$, and how the linear expansion is found. 


\subsubsection{System Observability}

For linear time invariant systems described by equation (2.4) and (2.5), the linear system with state vector $\mathbf{x}$ of dimension $n$ is observable if the observability matrix

$$
\mathbf{O}=\left[\begin{array}{c}
H \\
: \\
H A^{i} \\
: \\
H A^{n-1}
\end{array}\right]
$$

has matrix rank $n$ [19].

As $\mathbf{A}$ is an identity matrix in this research, the matrix $\operatorname{rank}$ of $O$ is the same as the matrix rank of $\mathbf{H}$. The linear system requires at least $n$ linearly independent measures to estimate a state vector $\mathbf{x}$ of size $n$.

Because we use (I)EKF to linearize the nonlinear system, the rank of $\mathbf{H}_{k}$ is used to determine the observability of the nonlinear system at time step $k$. The system is observable if the matrix rank of $\mathbf{H}_{k}$ is the same as $n$, the size of the state vector $\mathbf{x}$. 


\section{CHAPTER 3 PROBLEMS TO SOLVE}

\subsection{Problem Domain}

Dynamic resource management systems have two kinds of goals: they must maintain adequate quality of service (QoS), typically defined by service-level agreements (SLAs), and they may also seek to use the minimum resources to achieve the QoS requirements. The SLAs are normally represented as the constraints of the average user response times, the probabilities of exceeding the target delay, the average CPU utilizations and the throughputs. The resource cost is given by a cost function based on the resource used.

The primary target systems of this research are multi tier web-based service systems. The systems may include a number of clusters of web servers, application servers and database servers that may interact each other hierarchically. From the view of the client side, the users send requests to the web servers and expect to receive responses within an acceptable waiting time. From the view of the server side, the web server may reply to the requests directly, or query the databases via the application servers etc. For different number of users, the resources on the server side must be configured properly to achieve the users' QoS requirements. A typical example is the data center which is responsible for maintaining a number of applications or services with groups of computers as servers. The computers could be used for different applications by installing different software packages. Different applications may have different peak times. Furthermore there might be different classes of users to access the same application with different QoS requirements or priorities. The aim is to maintain a minimum number of computers to achieve the QoS requirements for all the applications with different classes of users using dynamic resource management strategies.

\subsection{Weakness of Existing Approaches to Resource Management}

In general a system may have several control variables and they may interact so that the choice of value should really consider them together. Single variable control 
strategies such as threshold control are less desirable.

The linearized dynamic control usually limits the maximum users or connections under overload conditions to make the system work around the designed operating point. The accepted users are guaranteed to have the expected QoS. However, the probability of being rejected may be very high with high arrival rates. It may be preferable to satisfy all the users by adding more resources instead of rejecting part of the users. The linearized dynamic strategy assumes the system works around the designed operating area. If the real operating point is far away from the designed operating area, the linearized model may not fit the system anymore. The controller built from the linearized model may not as good as expected.

The use of Markov decision process does deal with interactions with multiple control variables for nonlinear systems. However the state space explosion problem makes it only suitable for small systems.

Machine learning is a knowledge-free approach for resource management. It might be good if the system behaviours are close to those at learning phase. If the system varies from time to time, it is expensive or even impossible to use machine learning approach.

The queue based performance model control is able to configure the system with multiple control variables to meet the QoS requirements. It is scalable and suitable for nonlinear systems. Most of the work used static models which are not suitable for highly dynamic system. Recently, a few researches used inference or approximation to estimate the model parameters in queueing network models [31][54]. However, none of the existing researches updated the performance models in layered systems with software components.

\subsection{The Choice of Using LQN Performance Model}

The target system of this thesis is the multi tier enterprise system using J2EE architecture. In this type of system, the software components may be as important as the hardware devices. The simultaneous resource possession may result in the bottlenecks of a system. The traditional queueing network model is not able to model these features. LQN model has the ability to model the software components and simultaneous resource 
possession. It has been used in multi tier system to identify bottlenecks, control the number of processor replicas for provisioning, tune the number of server threads to ease the bottleneck, adjust priorities and reallocate processes for QoS requirements As an extension of queueing network performance model, it has advantages of queueing network model approach over other approaches such as the scalability and ability to control multiple variables for nonlinear systems simultaneously.

The LQN performance model is the best choice for this research.

\subsection{The Necessity of Tracking the System Changes}

It is well known that some of the parameters (e.g. execution demands) of the LQN model are difficult to calibrate. It is impossible or at least very expensive to measure those parameters directly.

Furthermore even if the parameters could be measured or estimated with experiments, the values of those parameters may change from time to time in real systems. In some cases, the dynamic behavior is so important that a static performance model can not describe the system accurately. For example:

1. In order to improve the performance of the Java virtual machine (JVM), dynamic optimization techniques have been applied to design JVM. It has been reported that if the JVM finds a loop with a big loop count, the codes related to this loop will be recompiled. The new code could be executed 10 times faster after recompiling.

2. An online e-commerce website may sell new items from time to time. The table size of the database will increase gradually. This may cause longer search time months later. The longer search time may affect the system behavior significantly if the table size is over a threshold.

3. Some types of transactions like browsing consume mainly the resources of the web servers, while other types like purchasing consume both the resources of the web servers and database servers. Different ratio of transaction types may affect the system performance.

4. User traffic might be much higher on peak time due to different reasons (e.g. an 
important sport game for a sport site, a political accident for a news site.)

In those cases, the systems must be updated in time. The tracking filter technique is able to estimate "hidden" model parameters (e.g. execution demands) from the available measurements. The parameters could be updated dynamically by monitoring the measurable quantities continuously.

\subsection{Solution Framework}

The dynamic resource management using performance model needs to solve following problems:

1. Generate a performance model structure for the real system.

2. Build a performance model with model parameters close to those of the real system

3. Update the model parameters while the real system changes

Solving these problems is time consuming and needs expertise. The goal of this research is to provide a framework that is able to solve those problems with automation. Then the resource management decisions could be made to achieve the QoS requirements by comparing the configurations of the performance model

Figure 1.2 shows the framework of the architecture. The System and Application block represents the real system or application we are going to control. The Performance Model block is the abstraction of the system or application. It is an LQN performance model that approximates the system characteristics in this research. The model building is separated as 2 parts: extract the model structure (problem 1), build and update the model parameters (problem 2 and 3). The Model Structure Extractor is used to construct the structure of the LQN model. The construction could be based on system design diagrams and the transaction traces across the components. The parameters of the LQN model are achieved and updated in 3 ways. It could be monitored by the System Monitor directly, calculated from Model Structure Extractor or estimated and updated by the Tracking Filter. The System Monitor block contains some tools to measure available system and performance metrics. The Model Structure Extractor block records all the transaction information that provides the information related to the visit counts. The Tracking Filter 
block is a tracking filter which estimates and updates indirect system variables from direct system variable via the System Monitor. The iterative extended Kalman filter (IEKF) is chosen as the tracking filter for this research. With a proper performance model and model parameters, the Decision Maker block is able to reconfigure the system or application under different workload conditions, available resources and QoS requirements.

In this research the change of the model parameters (e.g. user workloads and service demands) are assumed to be slow (e.g. take minutes), which makes the performance estimation feasible. For each time step of the tracking filter, it assumes to have enough data to provide adequate accuracy (e.g. confidence interval is less than $10 \%$ of the value). There are available tools in System Monitor and Model Structure Extractor block to provide real time data to the Performance Model block. 


\section{CHAPTER 4 MODEL STRUCTURE}

\section{EXTRACTOR}

Model based performance analysis needs to build a performance model. It is time consuming and needs expertise to build a precise model for a real system. The difficulties of the model building impede the use of the performance model in real systems. In this research, the model building will be automated to some degree in the framework proposed in the previous chapter. The model building is separated to 2 parts: to extract the initial model structure and to estimate the model parameters. This chapter focuses on the Model Structure Extractor block in the framework that generates the LQN model structure from the system architecture diagrams and the transaction traces.

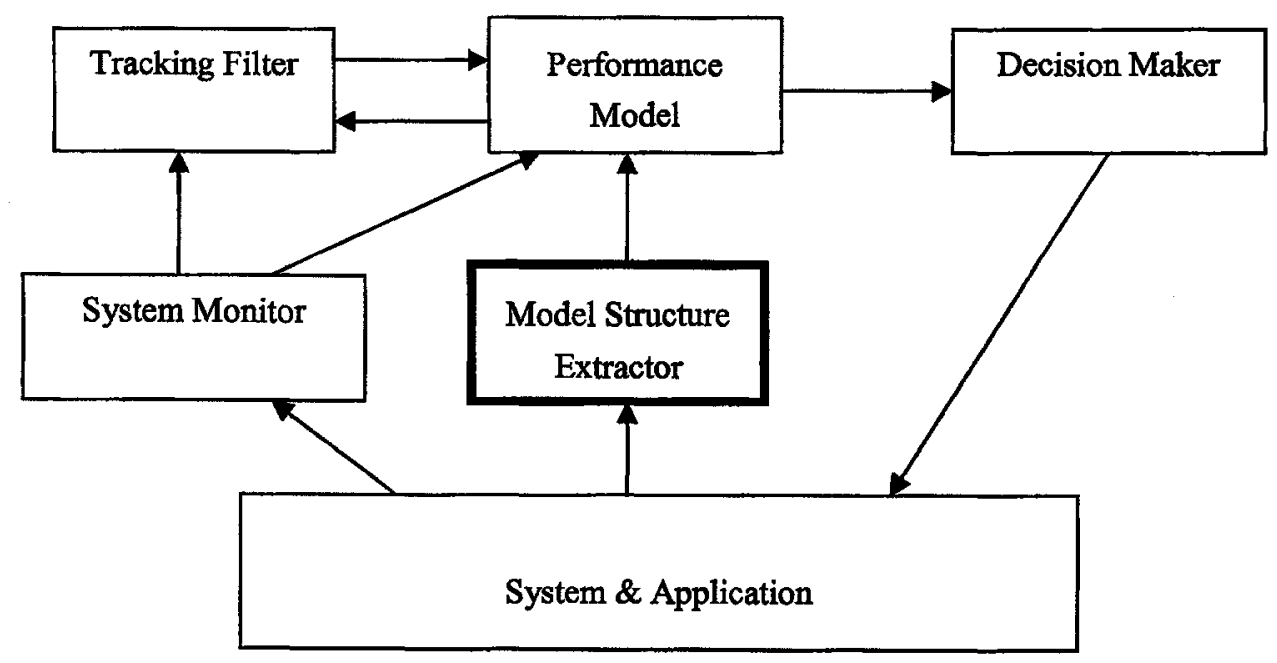

Figure 4.1 The Architecture of the Framework

The LQN model structure extraction consists of 2 parts: component determination and interaction determination. 


\subsection{Components Determination in the Performance Model}

The components of the model must be determined before the model generation. It includes the hardware and software components and host relationships, which are mapped to the tasks, entries and hosted processors in the LQN semantics. This information is derived from the system architecture diagrams or imported from system design files (e.g. deployment diagrams and class diagrams in unified model language (UML)). Generally, a computer or a node in the system architecture diagram is mapped to an LQN processor. A computer cluster is mapped to a processor with replicas. A software component in the architecture diagram is mapped as a task in the LQN model. The multiple threads of the software component could be represented as a task with multi servers. A software component may have different functions or methods. The functions or methods are mapped to the entries. Table 4.1 lists the component mapping between the real system and the LQN model.

\begin{tabular}{|c|c|}
\hline In System & In LQN Model \\
\hline Computer or Node & Processor \\
\hline Computer Cluster & Processor with Replicas \\
\hline Software Component or Process & Task \\
\hline Process with Multiple Threads & Task with Multi Servers \\
\hline Function or Method & Entry \\
\hline
\end{tabular}

Table 4.1 The Mapping between the System and the LQN Model

Generally the system architecture diagram and deployment diagram in UML provide information of the hardware devices in the system. The LQN processor and task information can be derived from them. The class diagram in UML provides information of software component in the system. The LQN task and entry information can be derived from it.

Figure 4.2 is the system architecture diagram of a distributed application and Figure 4.3 shows its related interactions. In Figure 4.2, each separated green (dark) block is considered as a LQN processor. Those processors were named as ClientCPU, App1CPU, $A p p 2 C P U, D b 1 C P U$. Each yellow (light) block is considered as a task. The hosts of the tasks are the processors they are within. The host relationship could be easily identified from Figure 4.2. Each separated yellow (light) block in Figure 4.3 is a service or function 


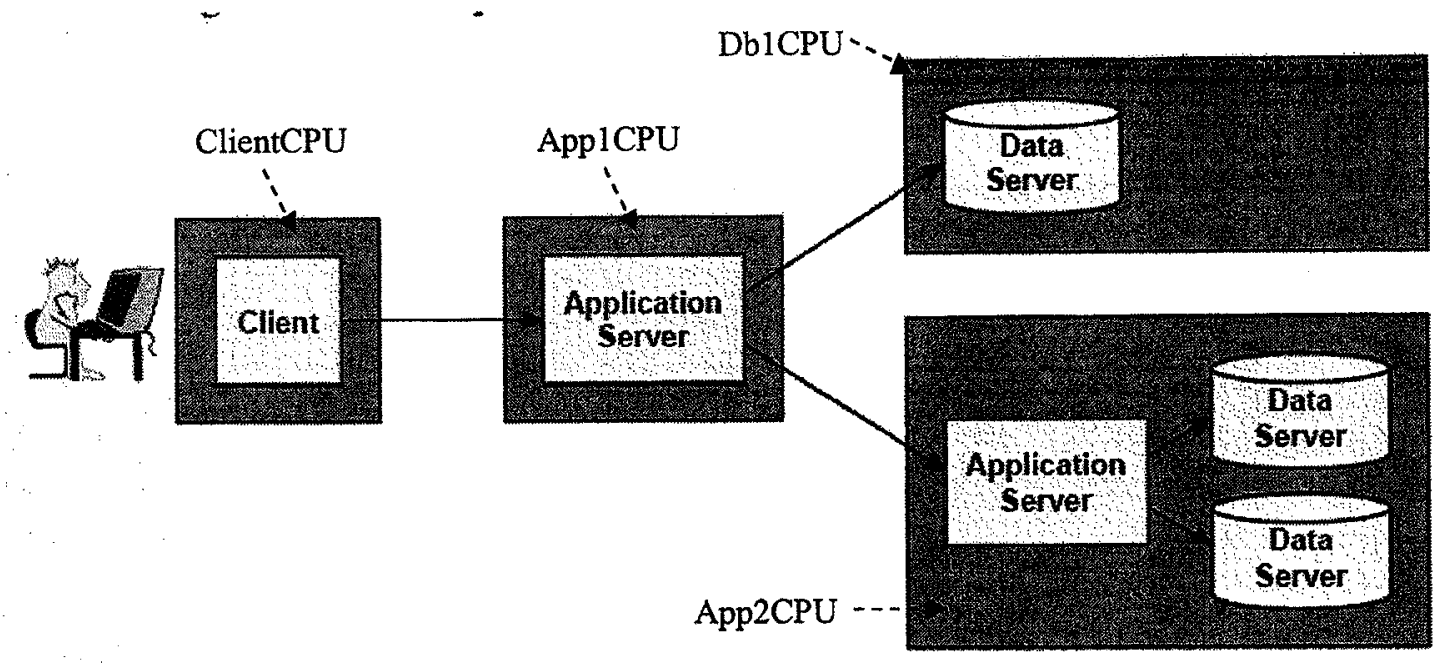

Figure 4.2 A Common Distributed Application Architecture [38]

which is considered as an LQN entry. Some related entries (e.g. query and update) are assigned to the same task, as they are the functions belong to the same software component (task).

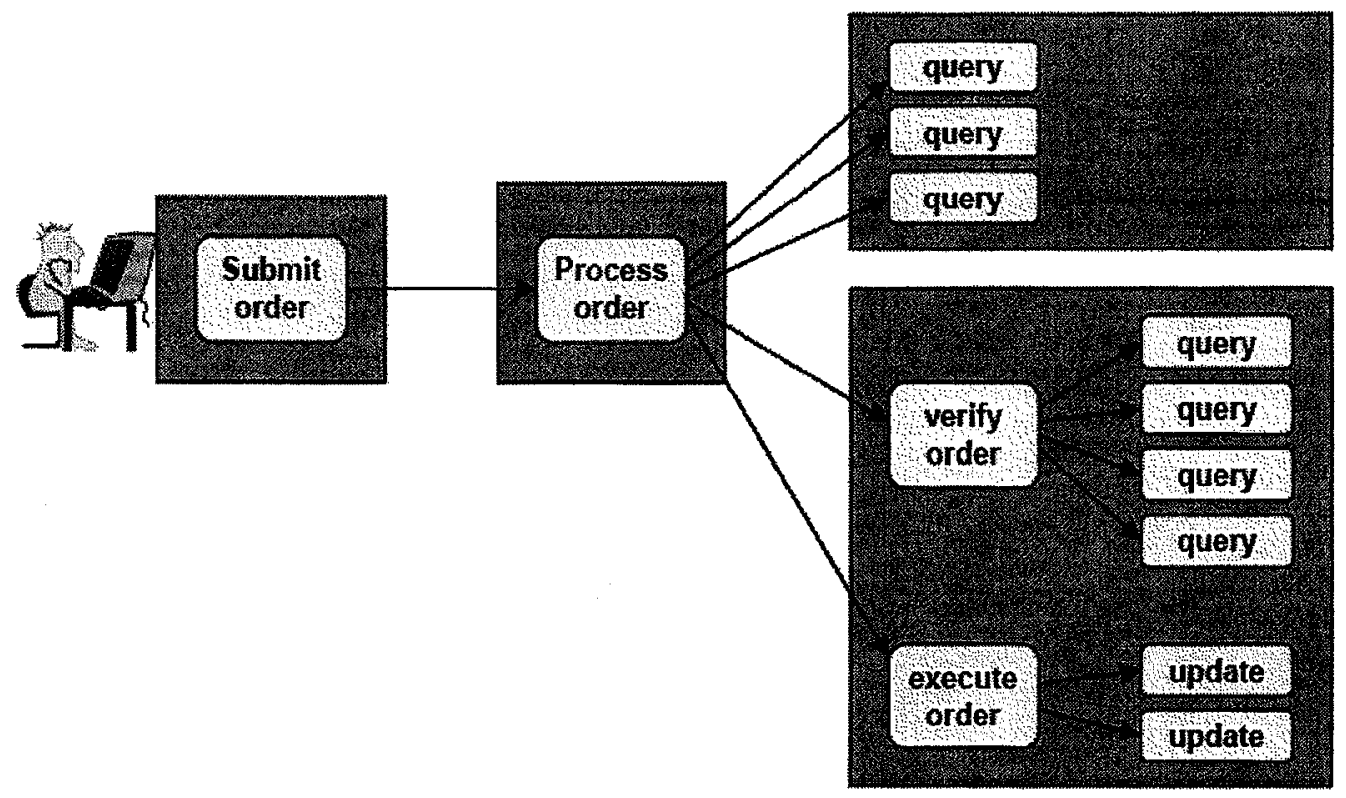

Figure 4.3 An Example of Distributed Transaction [38]

Table 4.2 shows the LQN processors, tasks and entries mapped from Figure 4.2 and Figure 4.3. In the column of Task and Entry, the name in parenthesis is the name shown 
in Figure 4.2 and Figure 4.3 while the other one is the name used in the LQN model. In LQN model, the name of Task or Entry must be unique.

\begin{tabular}{|l|l|l|}
\hline Processor & Task (Name in Figure 4.2, Figure 4.3) & Entry (Name in Figure 4.2, Figure 4.3) \\
\hline ClientCPU & Client (Client) & SubmitOrder (Submit order) \\
\hline App1CPU & App1 (Application Server) & ProcessOrder (Process order) \\
\hline App2CPU & App2 (Application Server) & VerifyOrder (verify order) \\
\hline App2CPU & App2 (Application Server) & ExecuteOrder (execute order) \\
\hline Db1CPU & Db1 (Database Server) & Q1 (query) \\
\hline Db1CPU & Db1 (Database Server) & Q2 (query) \\
\hline Db1CPU & Db1 (Database Server) & Q3 (query) \\
\hline App2CPU & Db2 (Database Server) & Q4 (query) \\
\hline App2CPU & Db2 (Database Server) & Q5 (query) \\
\hline App2CPU & Db2 (Database Server) & Q6 (query) \\
\hline App2CPU & Db2 (Database Server) & Q7 (query) \\
\hline App2CPU & Db3 (Database Server) & U1 (update) \\
\hline App2CPU & Db3 (Database Server) & U2 (update) \\
\hline
\end{tabular}

Table 4.2 The Component Information for the System Shown in Figure 4.2 and Figure 4.3

\subsection{Interactions Determination}

After the component determination, the interactions between the components should be determined to generate the structure of the performance model. The frequencies of the interactions should be determined as well.

\subsubsection{Interaction identification from ARM}

Application Response Measurement (ARM) [67] is a standard for measuring transaction response times and transaction status of single system and distributed applications. Transactions instrumented with ARM identifies the interactions between the software components. It provides a way to observe and analyze the availability and performance of the system.

An application uses ARM by creating objects that implement ARM interfaces, and then invoking methods on these objects. The objects use start() and stop() methods to record the round trip response times of the objects. An object can also be programmed to receive a correlation token from its parent and pass a correlation token to its children. For example, in Figure 4.3, Submit Order passes a correlation token to Process Order after 
ARM instrumentation. An interaction between Submit Order and Process Order is then identified. By executing all the transactions, all the existed interactions can be extracted.

\subsubsection{Frequencies of the identified interactions}

Let's define $I$ as the set of components (entries in LQN semantics) determined in section 4.1, $Y_{i j}$ as frequency of the identified interaction from components $i$ to $j(i, j \in I)$ from ARM instrumentation. By recording the transaction traces for a period of time, it is able to get $N_{i}$, the count which component $i$ is visited during the measurement period, and $N_{i j,}$, the count which the identified interaction from components $i$ to $j$ during the

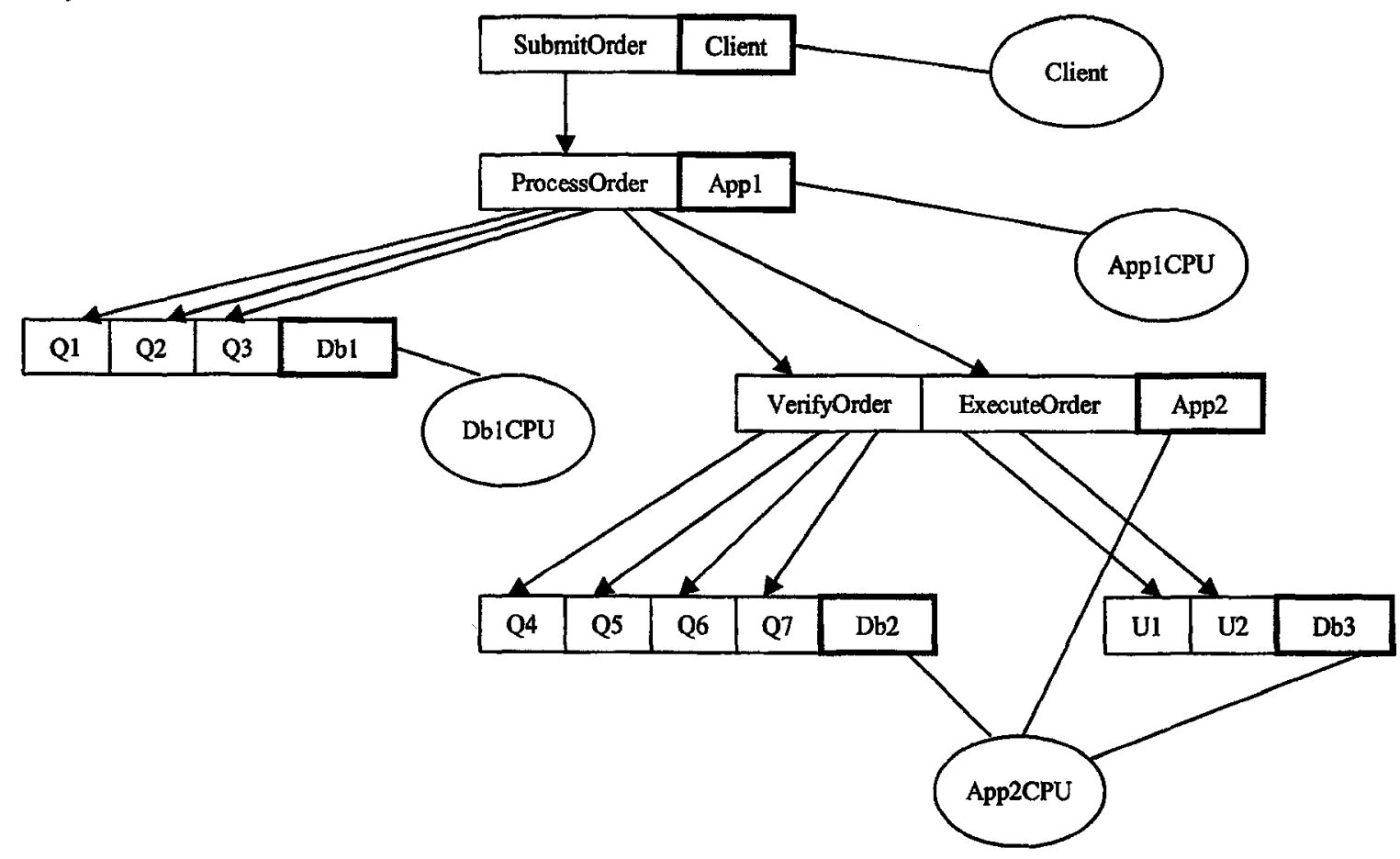

Figure 4.4 The Generated Structure of the LQN Model For the Application Shown in Figure 4.2 and Figure 4.3

measurement period. For a specific measurement period, we are able to calculate:

$$
Y_{i j}=N_{i j} / N_{i}
$$

The interactions and their frequencies are then determined.

Figure 4.4 is the structure of the performance model generated from the application shown in Figure 4.2 and Figure 4.3. 


\section{Summary}

The generation of the structure of the LQN performance model is summarized as follows:

1. Determine the LQN processor, task and entry from the system architecture diagrams and UML diagrams (e.g. deployment diagram, class diagram).

2. Implement ARM instruments.

3. Extract component interactions and collect data $N_{i}$ and $N_{i j}$ by executing ARM transactions.

4. Calculate the frequency $Y_{i j}$ using Eq. (4.1) for the identified interaction from components $i$ to $j$

Up to now, the service demand parameters of the LQN model are still missing. Those parameter values are difficult to be achieved from measurements directly. The tracking filter introduced in the next chapter will help to estimate those demand parameters. 


\section{CHAPTER 5 TRACKING FILTER FOR}

\section{PERFORMANCE MODELS}

This chapter focuses on the Tracking Filter block Figure 5.1 in the framework.

Tracking filter is a technique to estimate the changing value of the system states and parameters, which might be difficult to observe, based on the related measurements. It can be applied to any systems.

When a QN or LQN performance model was applied to analyze software applications, it was usually assumed that workloads and model parameters were known and static. But the workloads and model parameters (e.g. service demands of the

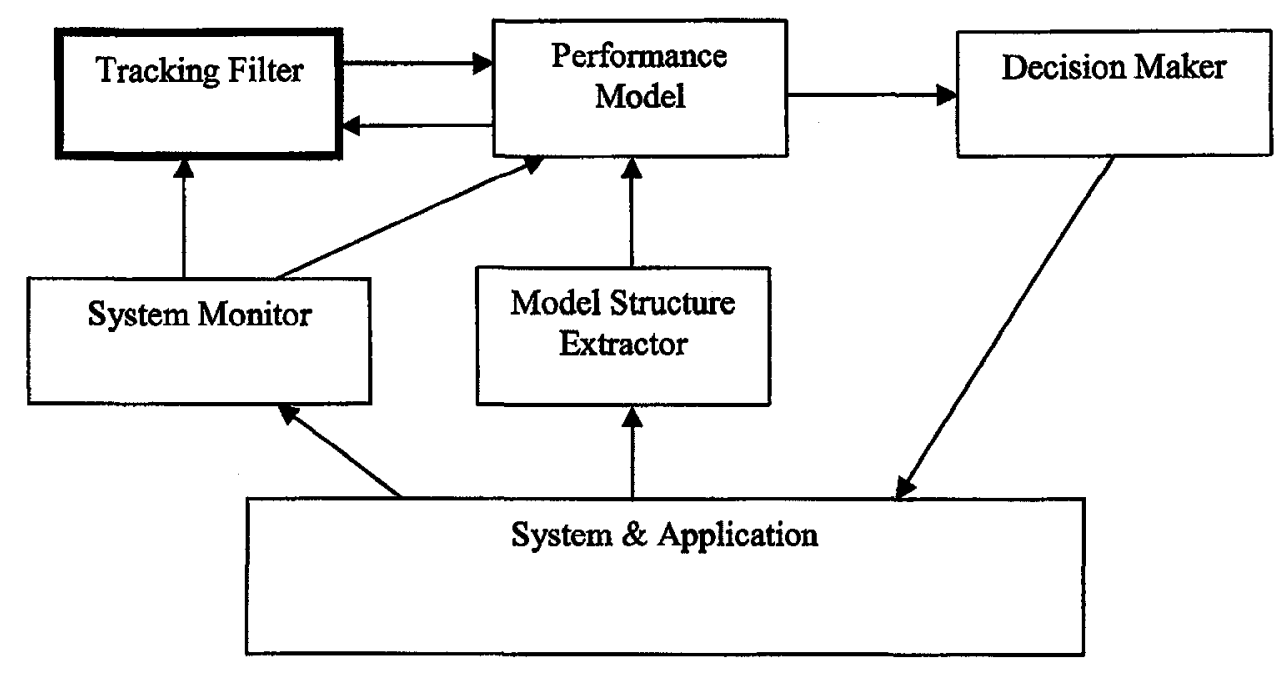

Figure 5.1 The Architecture of the Framework

queueing node) are sometimes difficult to measure directly in real systems. Furthermore, the real systems are usually dynamic which means that static model parameters might not be accurate enough when the system states change a lot. The advantages of using tracking filter in dynamic systems have been described in section 3.4. With the help of the 
tracking filter, workloads and service demands can be estimated and updated dynamically based on available system measurements (e.g. response times, utilizations, throughput etc.).

This chapter evaluates the tracking qualities of extended Kalman filter (EKF) and iterated extended Kalman filter (IEKF) with different settings which are important to apply the tracking filter technique to real systems. The evaluations will answer:

Which tracking filter is better for tracking the performance model?

How does the measurement step length affect the tracking quality?

How to set the filter parameter ( $\mathbf{Q}$ and $\mathbf{R}$ matrices in Eq. (2.7) and Eq. (2.8))?

How to choose the measurement sets?

How good will the tracking quality be?

These questions have not previously been addressed for estimating performance models.

In this thesis, the quality of per $f$ MARE $\left(x_{i}\right)=(1 / K) \sum_{k=1}^{K}\left|\hat{x}_{i, k-1}-x_{i, k}\right| / x_{i, k \text { s }}$ tracking was measured over a number of steps by its mean absolute relative error (MARE). For a tracked parameter $x_{i}$ in a simulated system, if $\hat{x}_{i, k}$ denotes the estimation of $x_{i}$ and $x_{i, k}$ denotes mean value of all the simulated samples during the $k$ th of $\mathrm{K}$ measurement steps, this gives

$$
\operatorname{MARE}\left(x_{i}\right)=(1 / K) \sum_{k=1}^{K}\left|\hat{x}_{i, k}-x_{i, k}\right| / x_{i, k}
$$

$\operatorname{MARE}\left(x_{i}\right)=(1 / K) \sum_{k=1}^{K}\left|\hat{x}_{i, k}-x_{i, k}\right| / x_{i, k}$

For a predicted performance measure $z_{i}$, the error was:

$$
\operatorname{MARE}\left(z_{i}\right)=(1 / K) \sum_{k=1}^{K}\left|y_{i, k}-z_{i, k}\right| / z_{i, k}
$$

where

$y_{i, k}$ and $z_{i, k}$ are the predictions and measurements of $h\left(x_{i}\right)$ at step $k$.

In real systems, the tracking filter will be used for prediction before the new measurements are available. Another set of error measures will be applied to indicate how good it is to use the tracking filter for prediction:

$$
f M A R E\left(x_{i}\right)=(1 / K) \sum_{k=1}^{K}\left|\hat{x}_{i, k-1}-x_{i, k}\right| / x_{i, k}
$$




$$
f M A R E\left(z_{i}\right)=(1 / K) \sum_{k=1}^{K}\left|y_{i, k-1}-z_{i, k}\right| / z_{i, k}
$$

$f M A R E\left(x_{i}\right)$ and $f M A R E\left(z_{i}\right)$ are called forward looking MAREs of the tracking parameters and measurement predictions. They emphasize the errors for the next step, when the model is applied to make decisions.

\subsection{The Use of EKF in a Queuing Network Model}

In [60], the EKF was applied to the queuing network model for the first time. The model (shown in Figure 5.2) has a Webserver node with its Disk and a separate node for $C G I$ application service. The response time of the $U$ sers includes the work done between visits.

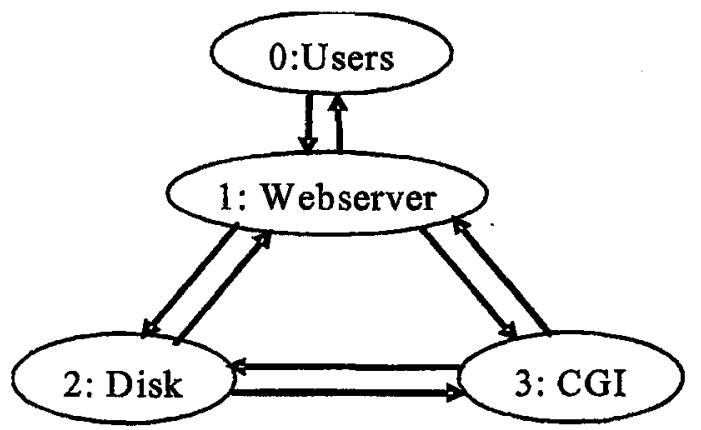

Figure 5.2 A Three-Node Queueing Model

The performance measures include:

$f=$ the throughput of requests from Users, per sec.,

$R T=$ the mean response time seen by users $=N / f$, where $N$ is the number of users

$R T(i)=$ the mean total delay at node $i$ per user request, assumed to include queueing and service,

$U(i)=$ the mean utilization of node $i$,

The tracking parameters are the service demands with exponential distributions: $\mathbf{x}=\mathbf{D}$ $=[D(1), D(2), D(3)]$. The default measurement set is $\mathbf{z}=[R T(1), R T(2), R T(3), f]$. The mean value analysis (MVA) [6] provides the exact values of $T(i)$ and $f$ from $D(i)$. The sensitivity matrix $\mathbf{H}$ can be inferred to calculate the derivatives $\partial R T(i) / \partial D(j)$ and $\partial f$ $/ \partial D(j)[60]$. 


\section{Parameter setting}

- The server demands change from $\mathbf{D}=[4,5,6]$ to $\mathbf{D}=[2,3,4]$ at time zero and remains constant.

- The performance model has the same structure and the filter begins at $\hat{x}_{0}^{-}=[4,5$, 6].

- $\mathbf{P}_{0}$, the estimated covariance of the initial estimate $\hat{\mathbf{x}}_{0}^{-}$, was chosen as the squares of the initial estimates, so $\mathbf{P}_{\mathbf{0}}=\operatorname{diag}(16,25,36)$.

- $\mathbf{R}=\operatorname{diag}(0.0109,0.0374,0.0745,0.0000154)$ where the values were the variance of the measurement vector $\mathbf{z}$ by running a simulation with the sampling length $S=$ 10000

- $\mathbf{Q}$ was set as an identity matrix.

\section{Experiments}

A number of experiments were implemented to investigate important issues applying EKF. The experimental results can be summarized as follows:

1. It is suitable to set covariance matrices $\mathbf{P}_{\mathbf{0}}, \mathbf{Q}$ and $\mathbf{R}$ as diagonal matrices for the tracking filter in practice.

2. A sample transient with $\mathbf{z}=[R T(1), R T(2), R T(3), f]$ shown in Figure 5.3 indicates that the parameter estimates change quickly to the real values, and remain with a small random variation. The throughput prediction error settles quickly to near zero. 


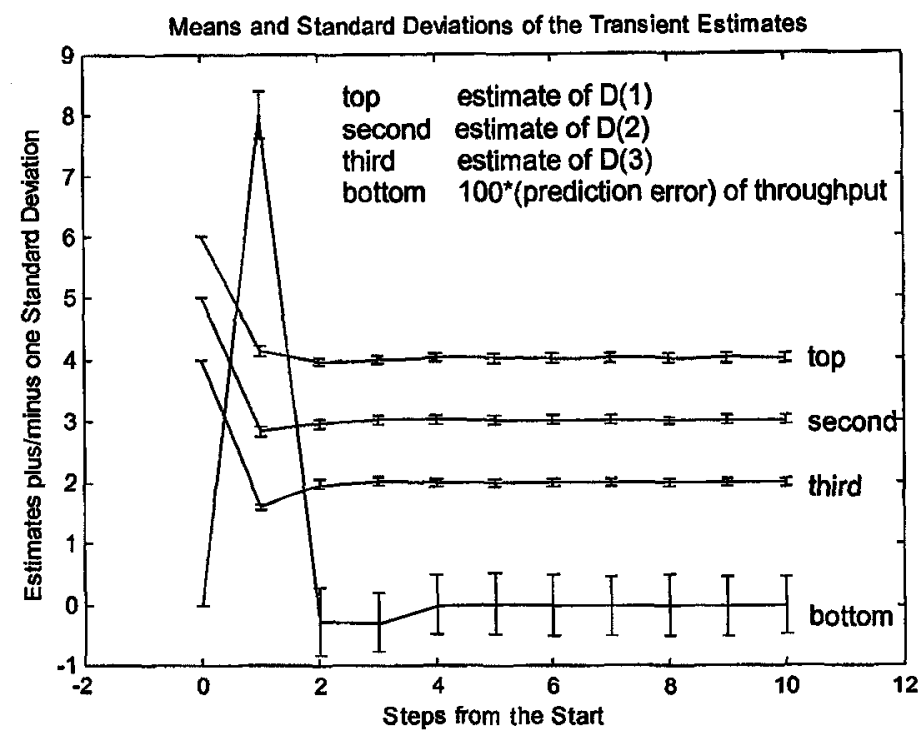

Figure 5.3 A Sample Transient, with error bars for one standard deviation

3. Figure 5.4 shows effect of scaling $\mathbf{R}$ on the steady state errors (after step 4 in Figure 5.3 where the model entered steady state). When $\mathbf{R}$ is increased, the filter tends to reduce its adaptation to the measurements, and its prediction quality suffers. $\mathbf{P}_{0}, \mathbf{Q}$ had almost no effect. The reason of $\mathbf{Q}$ having no effect is that the system is not actually drifting. The service demands remain the same all the time.

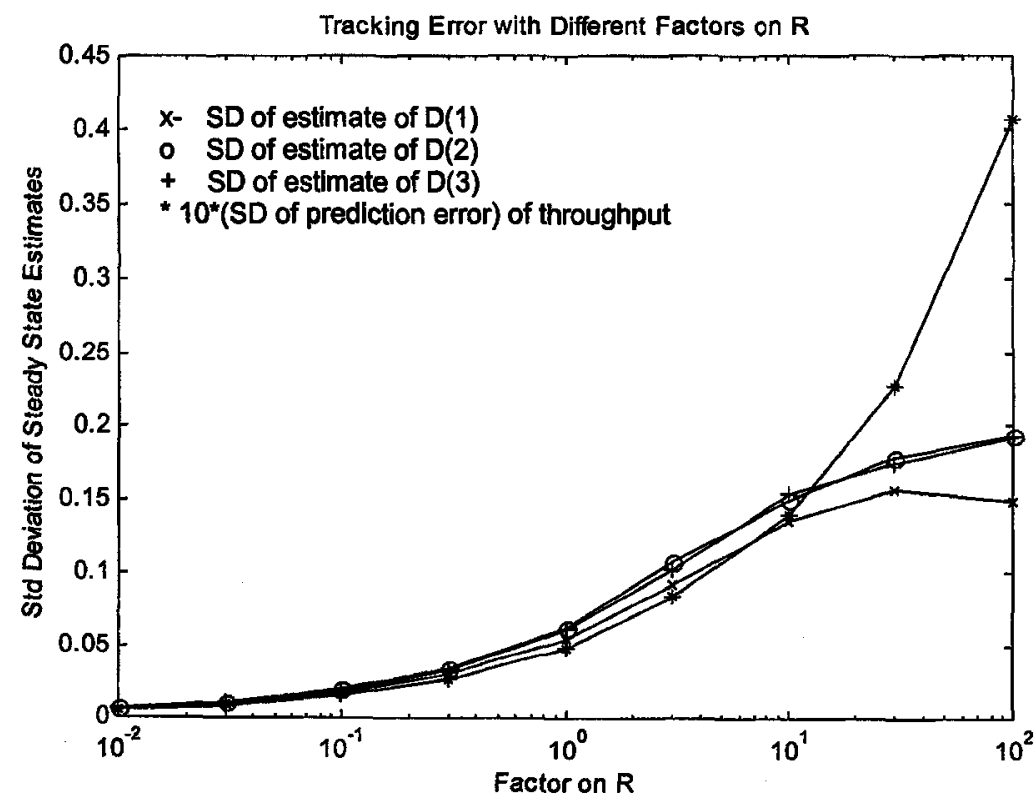

Figure 5.4 scaling the error variance matrix $R$ 
4. The filter is based on average measurements over a sampling step of $S$. The sampling step $S$ had a significant effect on the tracking errors. The longer the sampling step, the smaller the measurement errors and smaller tracking errors. As $\mathbf{R}$ is proportional to $1 / S$, Figure 5.5 is almost a mirror of Figure 5.4.

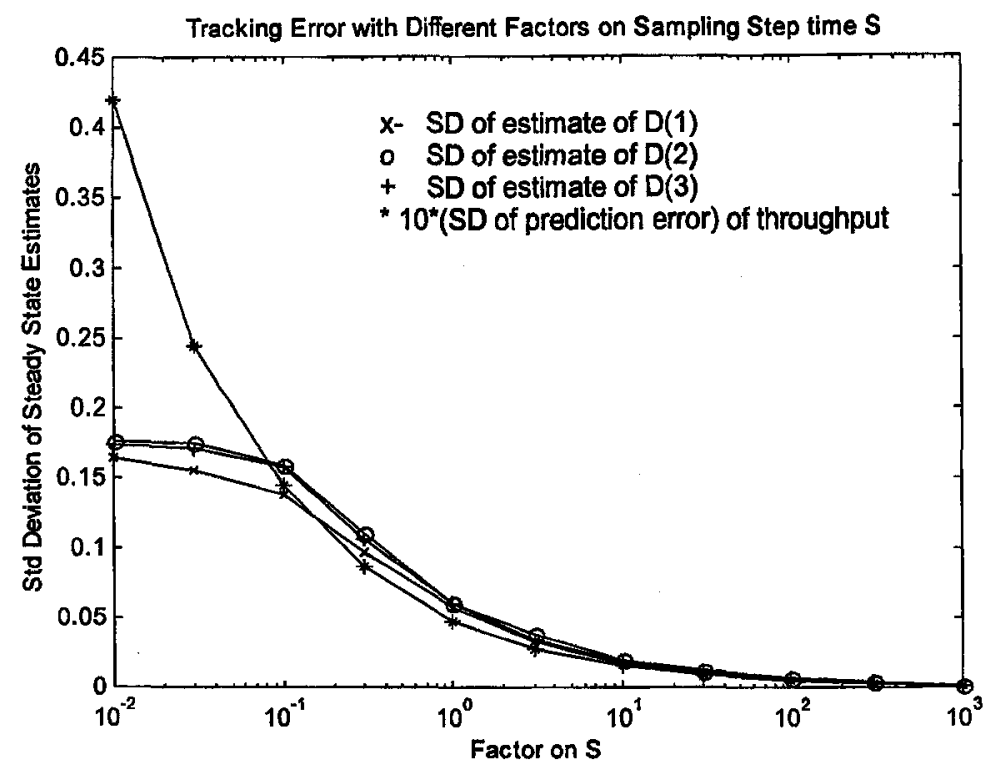

Figure 5.5 Scaling the sampling step time $S$

5. Extended Kalman filter (EKF) has its capability to fuse data of different types, such as delay, throughput, and utilization. It is not necessary to have direct data on every server in the system, but the observability matrix (Eq. (2.18)) must have a rank equal to the number of tracking parameters. If the rank of obervability matrix is smaller than the number of tracking parameters, some of the tracking parameters are not identified. Figure 5.6 shows a case with measurement $\operatorname{set} \mathbf{z}=$ $[U(2), f]$, which violates the identification condition. The errors for $D(1)$ and $D(3)$ are much larger than in Figure 5.5, while the errors for $D(2)$ are similar. The small errors for $D(2)$ reflect the better quality of information from the measured utilization $U(2)$. 


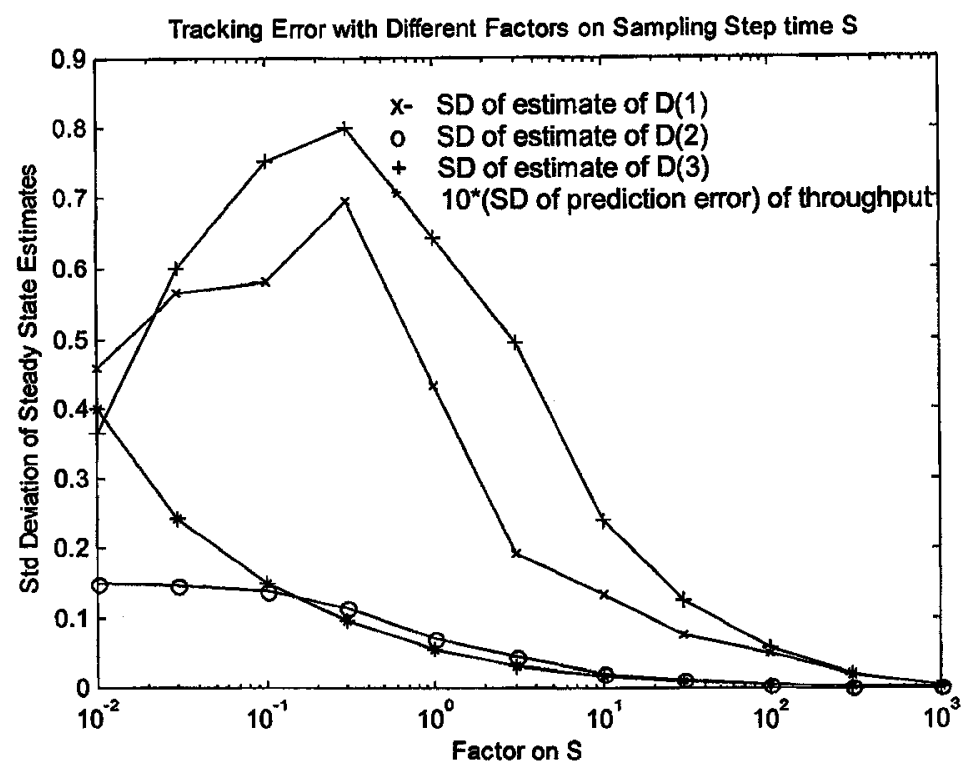

Figure 5.6 Variability of steady-state estimates with $\mathrm{z}=[U 2, f]$

6. Wrong models were used to test impact of a structural mismatch between the system and model, as performance models are always approximations. The filter was quite robust to the use of an approximate model, such as a model with fewer servers than the actual system. Figure 5.7 and Figure 5.8 show the transient and

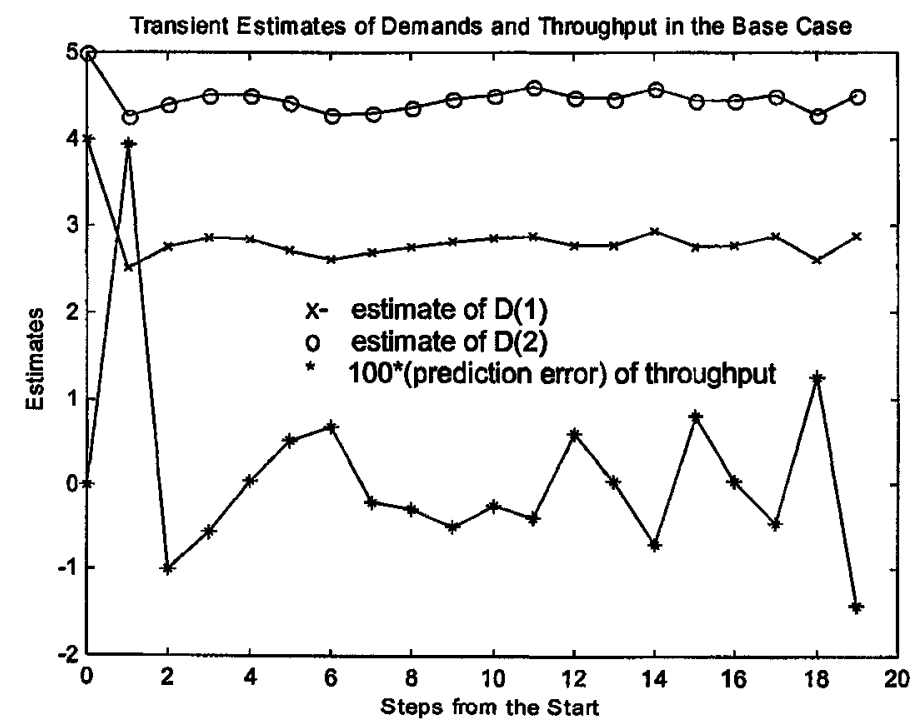

Figure 5.7 Typical transient of the estimates for a two-node model 


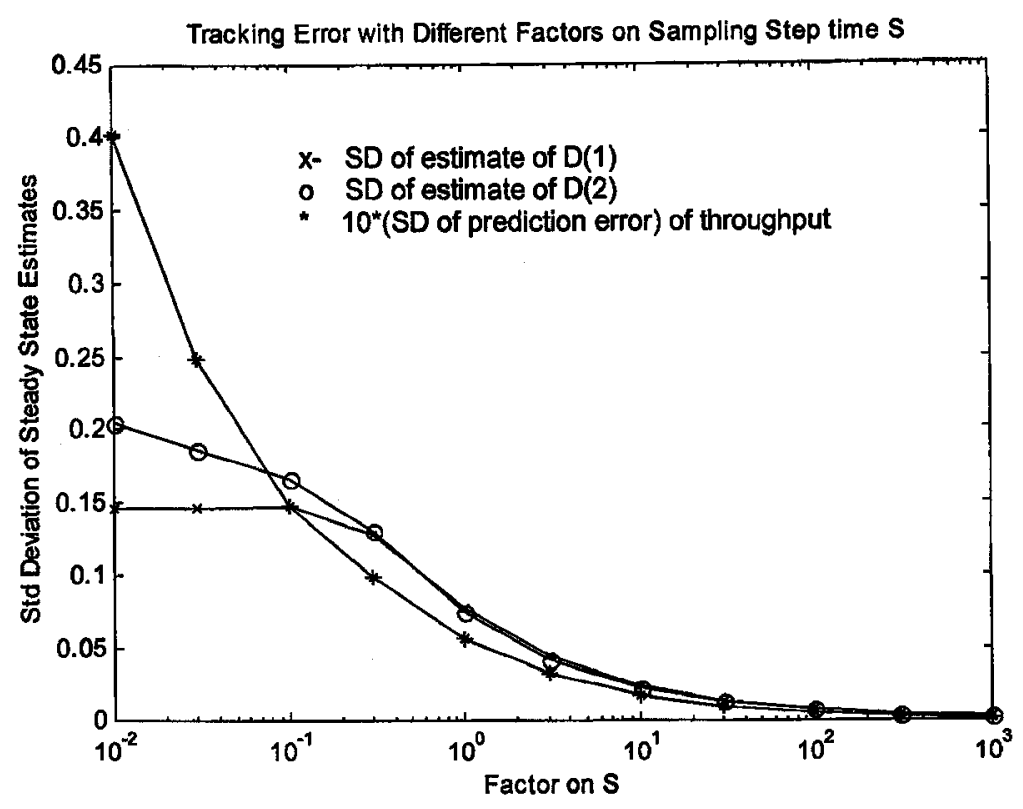

Figure 5.8 Standard deviations of estimates using a two-node model

standard deviations of estimate using a two-node (Node 1 and Node 2) model. The standard deviations are about the same as in Figure 5.5 while $D(1)$ and $D(2)$ are converged to 2.78 and 4.44. The throughput prediction errors in Figure 5.7 are only slightly larger than the one in Figure 5.3. The two-node model seems to predict and track as well as the original three-node model.

Overall the investigation was overwhelmingly positive about the potential of these estimators to track parameter changes.

\subsection{The Evaluation of (I)EKF in a LQN Model}

Based on the work described in previous section, the tracking filter was extended to layered queueing network (LQN) model in the rest of the thesis. Also, the iterated extended Kalman filter (IEKF) was applied as well as the extended Kalman filter (EKF).

This section studies the web-based application described in section 2.3. The LQN model of the application was shown in Figure 2.2. This model has been studied in [65] to evaluate the tracking quality of applying extended Kalman filter. In this section, a variant of Kalman filter called iterated extended Kalman filter (IEKF) will be used to estimate the model parameters in addition to the extended Kalman filter. The tracking quality of 
applying IEKF will be evaluated and compared with the one of applying EKF.

The default values of the model parameters are listed as follows:

$N$ : number of active users (default 100)

$Z$ : mean $U$ ser think time per request (default $1000 \mathrm{~ms}$ )

$S_{w}$ : mean WebServer CPU demand per user request (default $5 \mathrm{~ms}$ )

$S_{d}$ : mean Database CPU demand per database request (default $10 \mathrm{~ms}$ )

$M_{w}:$ number of WebServer threads (default 50)

$M_{d}:$ number of Database threads (default 1)

$N_{w}$ : number of WSProc replicas (default 1)

$N_{d}$ : number of $D B P r o c$ replicas (default 1)

A simulator using CSIM (see CsimSimulator in Appendix A) was implemented to simulate the system represented by the model shown in Figure 2.2. The statistics of simulation at each measurement step were considered as the measurements of the simulated system. The tracking filter maintains an LQN performance model by itself. At the end of each measurement step size, the tracking filter collects the measurements from the simulator. Those measurements are compared with the predictions solved by an analytic solver. The model parameters are then estimated and updated using Eq. (2.13) Eq. (2.17).

The tracking parameters were first set as $\mathbf{x}=[Z]$ or $\mathbf{x}=\left[S_{d}\right]$ where $Z$ is the user think time and $S_{d}$ is the service demand of the database server..

The tracking filter was driven by the measurement set $\mathrm{z}$ :

$$
\mathbf{z}=\left[R, U_{w}, U_{b}, U_{d}\right]
$$

where

$R$ is the user response time

$U_{w}, U_{b}$ and $U_{d}$ are the CPU utilizations of the WebServer, Database and Disk respectively.

\subsubsection{The determination of the filter parameters}

In the (iterated) extended Kalman filter (Eq. (2.11) - Eq. (2.17), the matrices $\mathbf{Q}$ and $\mathbf{R}$ 
capture knowledge or assumptions about the disturbances and the measurement errors, and they also influence how the filter reacts to new data.

Small values in $\mathbf{Q}$ describe an assumption that only small changes are expected, and lead to a small filter gain matrix $\mathbf{K}$ that can only adapt slowly. A large value of $\mathbf{Q}$ leads to large $\mathbf{P}$ and thus large gains that might overreact to measurement errors.

On the contrary, large values in $\mathbf{R}$ assume large measurement errors, and leads to small values in $\mathbf{K}$ that make the adaptation slowly. Small values in $\mathbf{R}$ make the filter adapt fast.

Both $\mathbf{Q}$ and $\mathbf{R}$ can be either static or adaptive. Static means the values in $\mathbf{Q}$ and $\mathbf{R}$ will not change after initialization. Adaptive means the values in $\mathbf{Q}$ and $\mathbf{R}$ may be different at each step. Both $\mathbf{Q}$ and $\mathbf{R}$ are static for simplicity at first. Adaptive $\mathbf{R}$ will be evaluated and discussed in section 5.6.

As mentioned in section 5.1, both $\mathbf{Q}$ and $\mathbf{R}$ can be assumed to be diagonal, with variance terms for the one step disturbances and the measurement errors, respectively.

Each diagonal element $Q_{i i}$ should be set to the square of an estimate of the magnitude of the changes to be tracked in parameter $x_{i}$ : For deterministic change of $x_{i}$ :

$$
Q_{i i}=\left(\text { maximum change of } x_{i}\right)^{2}
$$

For random change of $x_{i}$ with coefficient of variance $C_{i}$

$$
Q_{i i}=\left(C_{i} x_{i}\right)^{2}
$$

Before determining the values in $\mathbf{R}$, it is necessary to run a simulation to get a standard measurement step length $T^{*} . T^{*}$ was determined by giving a $95 \%$ confidence interval of $\pm 5 \%$ in the user response time measure $z_{l}$. For the system in Figure 2.1 with the default values of the parameters, $T^{*}=15.7 \mathrm{~s}$. From the asymptotic properties of the $t$ distribution, the confidence interval is 1.96 times the standard deviation.

Each diagonal element $R_{i i}$ should be an estimate of the variance of the measurement error in $\mathbf{z}_{\mathbf{i}}$. If the averaging measurement step time $T$ is large enough (which we shall assume is the case), the central limit theorem indicates that $R_{i i}$ should vary as:

$$
R_{i i}=\text { constant } / T
$$

For standard measurement step $T^{*}$ we can get

$$
R_{1 l}\left(T^{*}\right)=\left(0.05 z_{l} / 1.96\right)^{2} \text {. }
$$

For other values of $T$, the ratio of $T$ to $T^{*}$ is denoted by $\gamma_{T}$ : 


$$
\gamma_{T}=T / T^{*}
$$

and then, approximately:

$$
R_{I I}=R_{1 I}\left(T^{*}\right) T^{* / T}=\left(0.0255 z_{I}\right)^{2} / \gamma_{T}
$$

Further, it can be assumed that the confidence intervals of the other measures have similar accuracy. Thus:

$$
R_{i i}=\left(\begin{array}{ll}
0.0255 & z_{i}
\end{array}\right)^{2} / \gamma_{T}
$$

To demonstrate the ability of the filter to follow parameter changes, the system in Figure 2.2 was simulated with deterministic and random parameter changes (disturbance changes). A tracking filter was set up, based on the same model solved by an approximate analytic calculation with the LQNS solver.

The quality of tracking can be measured in two ways, by the performance prediction errors $\operatorname{MARE}\left(z_{i}\right), f M A R E\left(z_{i}\right)$ and by the parameter tracking errors $\operatorname{MARE}\left(x_{i}\right)$. fMARE $\left(x_{i}\right)$ defined by Eq. (5.1) - Eq. (5.4).

\subsection{Deterministic changes in parameter $Z$ or $S_{d}$}

Changes in a single parameter were tracked, with either $Z$ or $S_{d}$ i.e. $\mathbf{x}=[Z]$ or $\mathbf{x}=\left[S_{d}\right]$. $Z$ is the user think time which controls the user workload. $S_{d}$ is the service demand of the database which controls the resource consumption.

The tracking performance was recorded for a series of alternating step changes in value of two parameters. Each parameter was studied separately.

Case 1: The mean of the user think time $Z$ alternates between $500 \mathrm{~ms}$ and $2500 \mathrm{~ms}$ with a change every $471 \mathrm{~s}\left(30 T^{*}\right)$. The individual user think time is generated from an exponential distribution with the above means.

This creates a much larger arrival rate for small $Z$ than for large $Z$ (about 168/s when $Z$ $=500 \mathrm{vs} 39 / \mathrm{s}$ when $Z=2500$ ).

Different measurement step length $T \mathrm{~s}$ were used to investigate how the measurement step length affects the tracking quality. $T$ was set to $0.1 T^{*}, T^{*}$ and $10 T^{*}$ (making $\gamma_{T}=$ $0.1,1$ and 10 ). This gives estimate accuracy such that the $95 \%$ confidence interval in the mean user response time is roughly $\pm 15.8 \%, \pm 5 \%$ and $\pm 1.58 \%$ of the default mean value 
respectively according to Eq. (5.7). The total simulation time is $180 T^{*}$.

$\mathbf{Q}$ is a scalar, since there is just one parameter to track, and this is the square of the step change in $Z$ that is applied in going from 500 to 2500 . We can get

$$
\mathbf{Q}=(2500-500)^{2}=4,000,000
$$

from Eq. (5.5a).

$\mathbf{R}$ was a diagonal matrix with an element for each performance measure. It was calculated using Eq. (5.7)

Both EKF and IEKF were used for all the 3 different $T$ s.

The tracking quality measures were user response time prediction error $M A R E(R)$ and user think time tracking error $M A R E(Z)$

Table 5.1shows the $M A R E(R)$ and $M A R E(Z)$ for all the 6 combinations.

\begin{tabular}{|c|c|c|c|c|}
\hline & \multicolumn{2}{|c|}{ EKF } & \multicolumn{2}{c|}{ IEKF } \\
\hline & MARE(Z) & MARE $(R)$ & MARE $(Z)$ & MARE $(R)$ \\
\hline $\mathrm{T}=0.1 \mathrm{~T}^{*}$ & 0.163 & 0.116 & 0.162 & 0.067 \\
\hline $\mathrm{T}=\mathrm{T}^{*}$ & 0.065 & 0.067 & 0.044 & 0.028 \\
\hline $\mathrm{T}=10 \mathrm{~T}^{*}$ & 0.294 & 0.401 & 0.015 & 0.02 \\
\hline
\end{tabular}

Table 5.1 MARE(R) and $M A R E(Z)$ for deterministic change of $Z$

From Table 5.1, we can observe that

- The $M A R E(R)$ and $M A R E(Z)$ of using IEKF are always better than those of using EKF. When $T=0.1 T^{*}$, The $M A R E(Z) s$ are very close. 


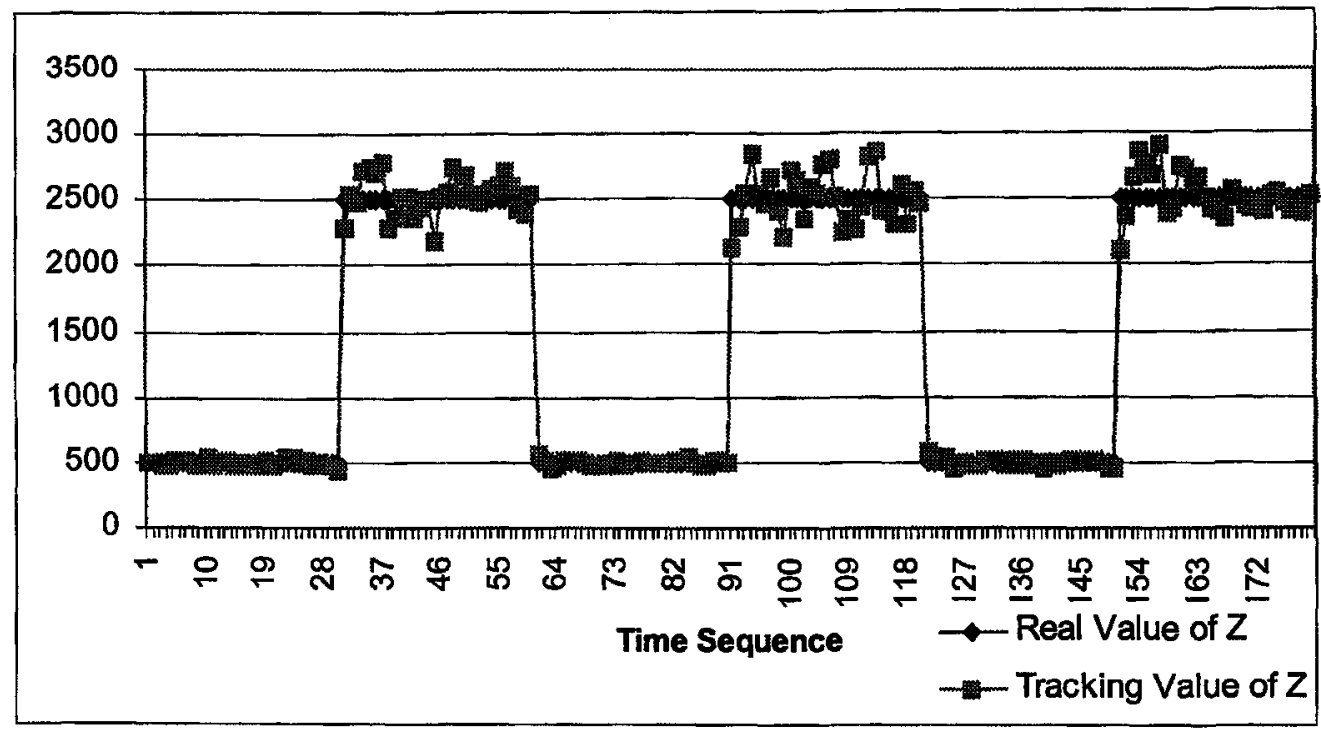

Figure 5.9 (Case 1) Tracking Performance for a Deterministic Sequence of $Z$ $\left(T=T^{*}\right)(\mathrm{IEKF})$

- In the case of IEKF, the tracking quality improves when $T$ becomes big. The larger the measurement errors (the measurement step length is smaller), the worse the tracking quality. For example, the MAREs when $T=T^{*}$ are much smaller than those when $T=0.1 T^{*}$. This phenomenon can be observed in Figure 5.9 as well. In Figure 5.9, the tracking error was visibly greater for $Z=$ $2500 \mathrm{~ms}$. When $Z=2500 \mathrm{~ms}$, there are fewer response in the averaging period, so the variances of the measurements are larger than those when $Z=500 \mathrm{~ms}$.

- In the case of EKF, MAREs in the case of $T=0.1 T^{*}$ are bigger than the MAREs when $T=T^{*}$, whose trend is similar to the case of IEKF. Figure 5.10 is similar to Figure 5.9 which has larger tracking error when $Z=2500 \mathrm{~ms}$. One difference is that EKF took 3 or 4 step to catch the change from $500 \mathrm{~ms}$ to $2500 \mathrm{~ms}$ while IEKF took 1 step only.

- However, when $T=10 T^{*}$, the MAREs of using EKF are much bigger than expected. There errors are supposed to be smaller as the larger measurement step length provides smaller measurement errors. There must be other factors 


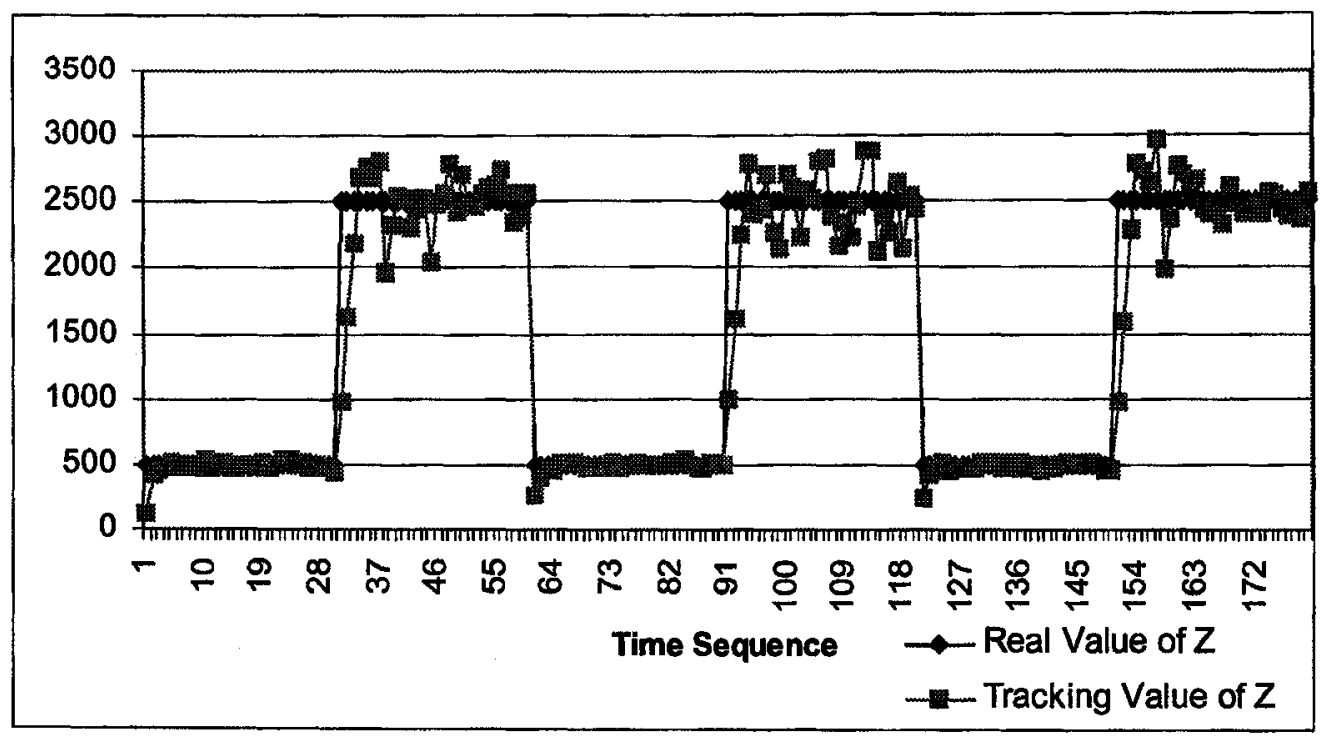

Figure 5.10 (Case 1) Tracking Performance for a Deterministic Sequence of $Z$ $\left(T=T^{*}\right)(\mathrm{EKF})$

besides the measurement errors that affect the tracking quality of EKF. The hidden factors can be shown clearly in Figure 5.11 and Figure 5.12 with the transients of tracking using EKF and IEKF. Although the measurement error is quite small when $T=$ $10 T^{*}$, the changing rate becomes very fast relative to $T$. The EKF seems to need several steps to reach the new value, thus it is not able to keep up with fast changes (Figure 5.11). IEKF does not have this drawback. It is able to catch up the change in 1 step and the tracking quality is quite good (Figure 5.12). This explains why the tracking quality of IEKF is much better than EKF when $T=10 T^{*}$ 


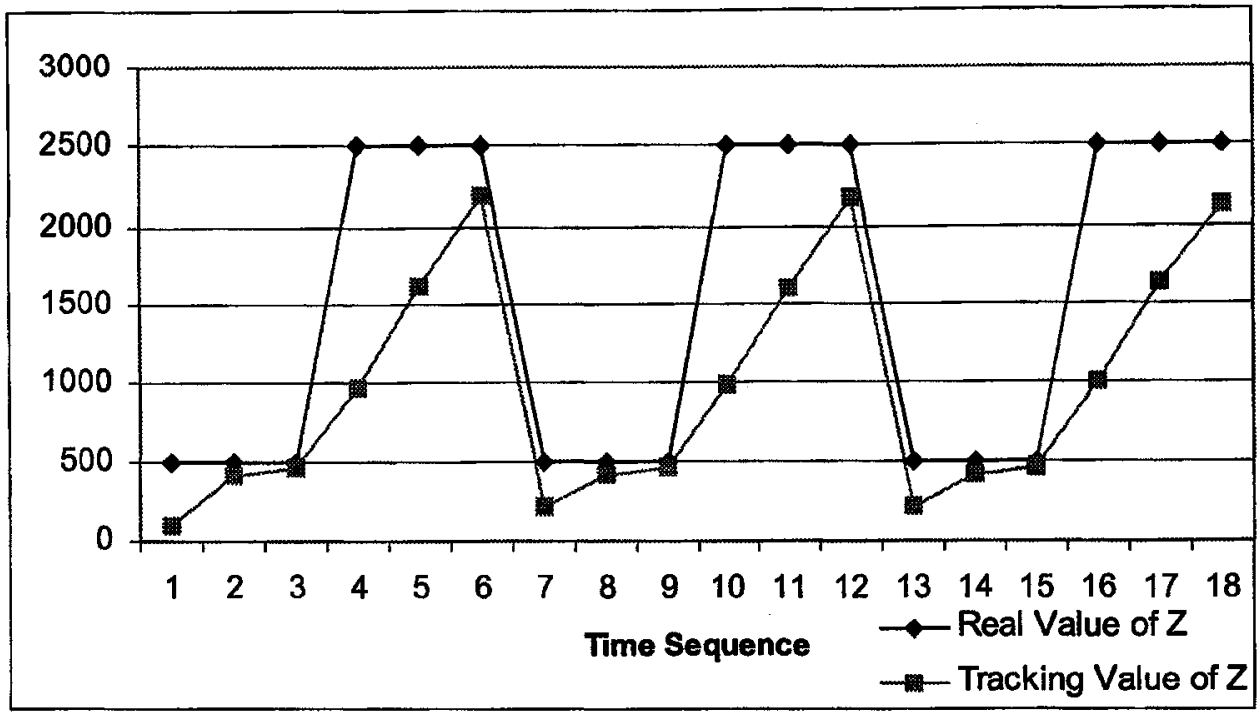

Figure 5.11 (Case 1) Tracking Performance for a Deterministic Sequence of $Z$ $\left(T=10 T^{*}\right)(\mathrm{EKF})$

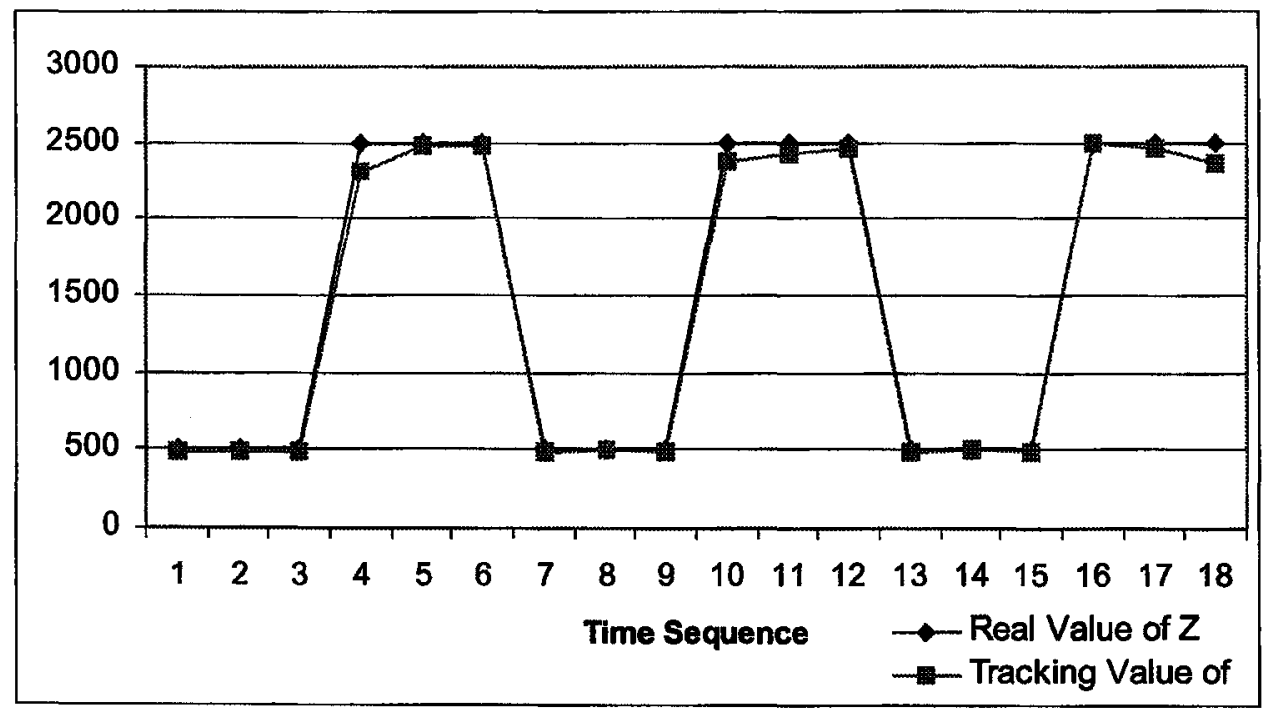

Figure 5.12 (Case 1) Tracking Performance for a Deterministic Sequence of $Z$ $\left(T=10 T^{*}\right)($ IEKF)

Case 2: In this case, the database demand $S_{d}$ (instead of user think time $Z$ ) alternates between $10 \mathrm{~ms}$ and $40 \mathrm{~ms}$, with changes every $471 \mathrm{~s}\left(30 T^{*}\right)$. At the lower value, the system is lightly loaded; the higher value creates a significant load at the database, with a queuing delay that blocks some application threads.

Other experiment conditions were set the same as in case 1 except $Q$ was set to 900 44 
and $\operatorname{MARE}\left(S_{d}\right)$ represents the tracking error of the $S_{d}$

\begin{tabular}{|c|c|c|c|c|}
\hline & \multicolumn{2}{|c|}{ EKF } & \multicolumn{2}{c|}{ IEKF } \\
\hline & MARE(Sd) & MARE $(R)$ & MARE(Sd) & MARE(R) \\
\hline$T=0.1 T^{*}$ & 0.147 & 0.160 & 0.152 & 0.029 \\
\hline$T=T^{*}$ & 0.083 & 0.206 & 0.049 & 0.012 \\
\hline$T=10 T^{*}$ & 0.330 & 1.176 & 0.019 & 0.004 \\
\hline
\end{tabular}

Table 5.2 MARE $(R)$ and $M A R E\left(S_{d}\right)$ for deterministic change of $S_{d}$

Table 5.2 shows that the MAREs of using IEKF are generally smaller than the ones using EKF. The MAREs of using EKF when $T=10 T^{*}$ are much larger than expected. Those results are similar to those of Table 5.1 for case 1 . The analysis in the previous case works in this case as well. IEKF is able to catch up the change in 1 step while EKF may take several steps. This feature deteriorates the tracking quality of EKF when the change rate is fast relative to $T$. In the case of IEKF, the MAREs become small when the measurement step length increase.

Figure 5.13 shows the transients of tracking which corresponds to Figure 5.9 for case1. In Figure 5.13, the larger tracking errors are evident for the larger value of $S_{d}$, which corresponds to a heavier load (as opposed to the case 1 in which the larger value of $Z$ gives the lighter load). This time the number of responses in an averaging period decreases with heavy load which lowers the arrival rate.

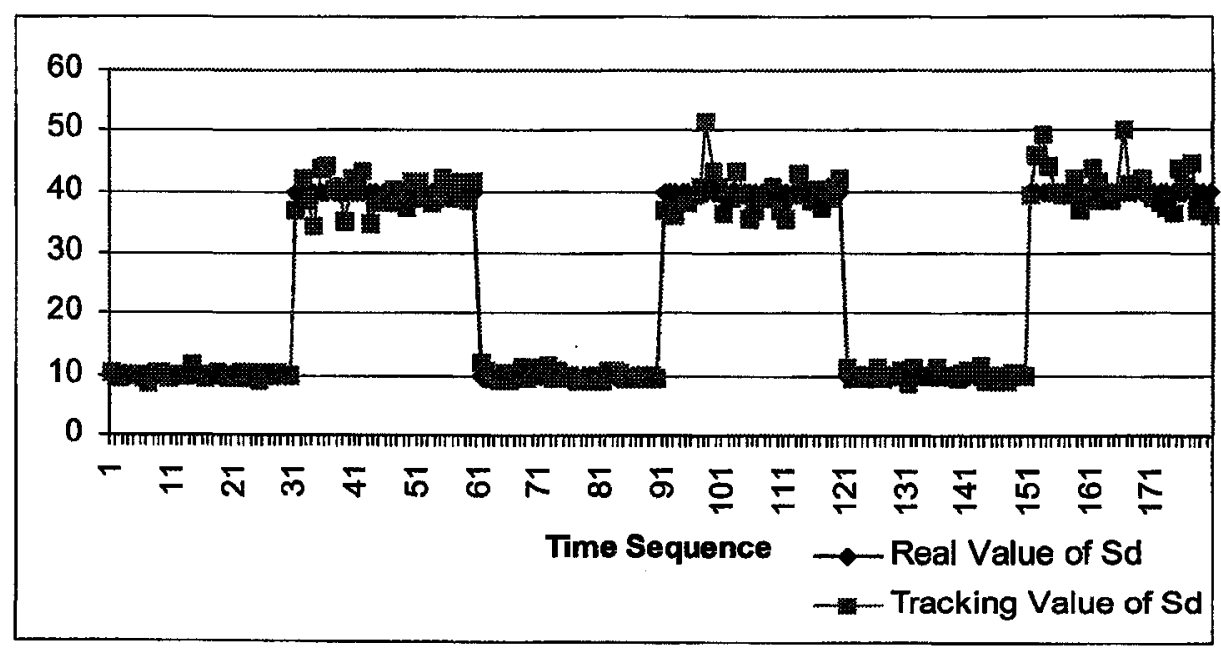

Figure 5.13 (Case 2) Tracking Performance for a Deterministic Sequence of $S_{d}$ $\left(T=T^{*}\right)($ IEKF)

Summary of case 1 and case 2 
From the results of case 1 and case 2, it was found that:

- Overall, IEKF provides better tracking quality than EKF. The tracking quality of EKF was extremely bad when the change rate is fast relative to $T$.

- IEKF tends to have smaller MAREs with smaller measurement errors.

\subsection{Random changes in parameters $Z$ or $S_{d}$}

In this section, a random change process was generated in the simulation, with step changes of a parameter value occurring at randomly chosen. The change process was applied to one parameter at a time either $Z$ or $S_{d}$.

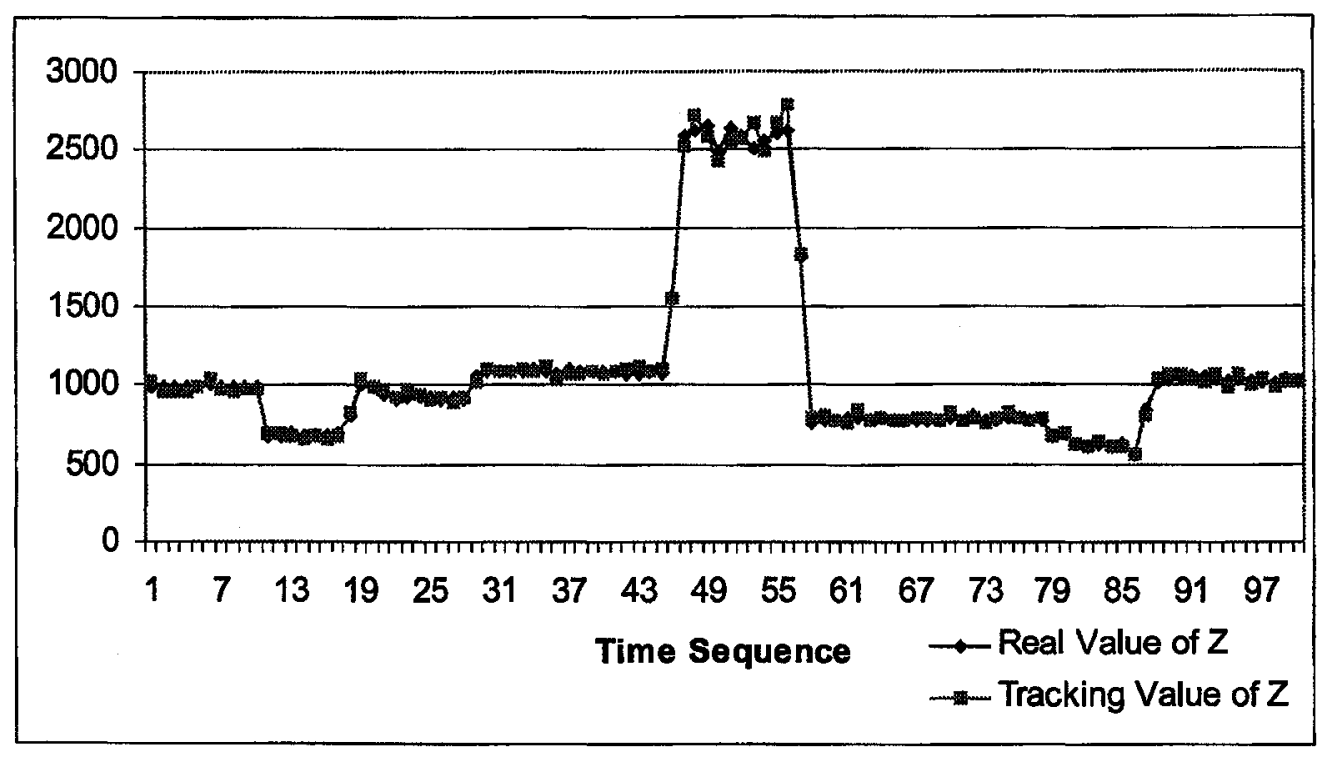

Figure 5.14 A Trace Fragment of Tracking Random Changes of $Z$ (IEKF)

Figure 5.14 shows a fragment of a trace of the filter tracking random changes in the mean user think time $Z$. Generally, EKF follows a change within a few steps while IEKF is able to follow a change in one step. The tracking errors are relatively large when $Z$ is big. The reason is the same as that explained in case 1.

\section{Change Process}

At each change instant, a new value of the parameter $\left(Z\right.$ or $\left.S_{d}\right)$ was chosen independently according to a shifted hyper-exponential distribution [4]:

$$
M=P /(1+C)+\operatorname{Hyper}\left(P^{*} C /(1+C),\left(P^{*} C\right)^{2}\right)
$$


where $M$ is the new value of the parameter (e.g. $Z, S_{d}$ ),

$P$ and $C$ are the mean value and coefficient of variation of the distribution of the parameter value

Hyper $\left(P^{*} C /(1+C),\left(P^{*} C\right)^{2}\right)$ is the hyper exponential distribution with mean $P^{*} \mathrm{Cl}$ $(1+C)$ and variance $\left(P^{*} C\right)^{2}$

$M$ has the mean value of $P$ and coefficient of variation close to $C$. It has a feature that it always has a lower limit value. It avoids very small values of $Z$ which might make the analytic solver give errors larger than usual. When a new value of $X$ is generated, it will be assigned as the mean value of the parameter until the next change occurs.

\section{Case 3: Parameters (Q and $R$ ) Tuning}

The filter tuning parameters $\mathbf{Q}$ and $\mathbf{R}$ might affect the way the filter reacts. Small values in $\mathbf{Q}$ make the filter conservative, as it assumes only small changes are possible in a single step. Small values in $\mathbf{R}$ make the filter track more aggressively, as it assumes that the measurements are accurate and therefore the filter must react in order to explain them. We must learn how to set these parameters, and also it is important to understand how sensitive the whole filter process is to their values.

To investigate these effects, the entries of $\mathbf{Q}$ and $\mathbf{R}$ were multiplied by factors denoted as $Q F a c$ and $R F a c$ respectively. These two factors were varied over two orders of magnitude. The expressions used for $\mathbf{Q}$ and $\mathbf{R}$ in Eq. (5.5b) and Eq. (5.7) became:

$$
\begin{aligned}
& Q_{i i}=\left((Q F a c) x_{i} C_{i}\right)^{2} \\
& R_{i i}=\left((R F a c)\left(z_{i}\right) 0.0255\right)^{2} / \gamma_{T}
\end{aligned}
$$

The experiments had the usual base values of $\gamma_{T}=4.0$ and $C_{i}=1.0$ and change rate of $40 T^{*}$. Both EKF and IEKF were used to estimate $Z$ or $S_{d}$ with different combinations of $Q F a c$ and $R F a c$. The simulation was applied 10000 steps for each combination of $Q F a c$ and $R F a c$.

The results in Table 5.3 - Table 5.6 show that the IEKF has smaller MAREs than the EKF in every case, just as in case 1 and case 2. It also agrees with the conclusion in [27] which indicates that IEKF is the most informative tracking filter for systems with linear process model and nonlinear measurement model. From the experiment results we 
obtained, we can conclude that IEKF is better than EKF to estimate system parameters. In the rest of the thesis, IEKF will be the default tracking filter and only results using IEKF will be provided and discussed.

\begin{tabular}{|c|c|c|c|c|c|c|c|c|c|c|}
\hline & \multicolumn{9}{|c|}{ QFac } \\
\hline & & 0.01 & 0.03 & 0.1 & 0.3 & 1 & 3 & 10 & 30 & 100 \\
\hline \multirow{9}{*}{ RFac } & 0.01 & 0.037 & 0.037 & 0.037 & 0.037 & 0.037 & 0.037 & 0.037 & 0.037 & 0.037 \\
\hline & 0.03 & 0.037 & 0.037 & 0.037 & 0.037 & 0.037 & 0.037 & 0.037 & 0.037 & 0.037 \\
\hline & 0.1 & 0.038 & 0.037 & 0.037 & 0.037 & 0.037 & 0.037 & 0.037 & 0.037 & 0.037 \\
\hline & 0.3 & 0.049 & 0.038 & 0.037 & 0.037 & 0.037 & 0.037 & 0.037 & 0.037 & 0.037 \\
\hline & 1 & 0.107 & 0.051 & 0.038 & 0.037 & 0.037 & 0.037 & 0.037 & 0.037 & 0.037 \\
\hline & 3 & 0.228 & 0.107 & 0.049 & 0.038 & 0.037 & 0.037 & 0.037 & 0.037 & 0.037 \\
\hline & 10 & 0.412 & 0.244 & 0.107 & 0.051 & 0.038 & 0.037 & 0.037 & 0.037 & 0.037 \\
\hline & 30 & 0.616 & 0.412 & 0.228 & 0.107 & 0.049 & 0.038 & 0.037 & 0.037 & 0.037 \\
\hline & 100 & 0.871 & 0.637 & 0.412 & 0.244 & 0.107 & 0.051 & 0.038 & 0.037 & 0.037 \\
\hline
\end{tabular}

Table 5.3 (Case 3) The MARE(Z), as RFac and QFac are varied using EKF

\begin{tabular}{|c|c|c|c|c|c|c|c|c|c|c|}
\hline & \multicolumn{9}{|c|}{ QFac } \\
\hline & & 0.01 & 0.03 & 0.1 & 0.3 & 1 & 3 & 10 & 30 & 100 \\
\hline \multirow{9}{*}{ RFac } & 0.01 & 0.044 & 0.044 & 0.044 & 0.044 & 0.044 & 0.044 & 0.044 & 0.044 & 0.044 \\
\hline & 0.03 & 0.044 & 0.044 & 0.044 & 0.044 & 0.044 & 0.044 & 0.044 & 0.044 & 0.044 \\
\hline & 0.1 & 0.042 & 0.044 & 0.044 & 0.044 & 0.044 & 0.044 & 0.044 & 0.044 & 0.044 \\
\hline & 0.3 & 0.038 & 0.042 & 0.044 & 0.044 & 0.044 & 0.044 & 0.044 & 0.044 & 0.044 \\
\hline & 1 & 0.029 & 0.036 & 0.042 & 0.044 & 0.044 & 0.044 & 0.044 & 0.044 & 0.044 \\
\hline & 3 & 0.039 & 0.029 & 0.038 & 0.042 & 0.044 & 0.044 & 0.044 & 0.044 & 0.044 \\
\hline & 10 & 0.061 & 0.041 & 0.029 & 0.036 & 0.042 & 0.044 & 0.044 & 0.044 & 0.044 \\
\hline & 30 & 0.088 & 0.061 & 0.039 & 0.029 & 0.038 & 0.042 & 0.044 & 0.044 & 0.044 \\
\hline & 100 & 0.113 & 0.091 & 0.061 & 0.041 & 0.029 & 0.036 & 0.042 & 0.044 & 0.044 \\
\hline
\end{tabular}

Table 5.4 (Case 3) The $M A R E(R)$ as $R F a c$ and $Q F a c$ are varied using EKF

The values in Table 5.5 and Table 5.6 also indicate that it is the ratio of QFac to RFac that is important, rather than the values of the parameters $\mathbf{Q}$ and $\mathbf{R}$. The tracking quality is almost the same for the same ratio of $Q F a c$ to $R F a c$. Also roughly, above the diagonal (when $\mathbf{Q}$ is too large, and the filter over-responds to measurement errors), the tracking quality is almost the same. On the other hand, when $\mathbf{Q}$ is too small (the filter is sluggish), the error increases steadily.

We can conclude that the tracking performance is somewhat insensitive to $\mathbf{Q}$ and $\mathbf{R}$. 
Around the ideal balance between $\mathbf{Q}$ and $\mathbf{R}$, there is a wide range (more than a factor of 100 up or down) in which the filter provides the same tracking quality.

The experiment results suggested that if we lack information to choose proper values for $\mathbf{Q}$ and $\mathbf{R}$, it is better to overestimate $\mathbf{Q}$ (rather than underestimate) relative to $\mathbf{R}$.

In the rest of the thesis, we set $Q F a c=1$ and $R F a c=1$ and use IEKF to estimate the system parameters.

\begin{tabular}{|c|c|c|c|c|c|c|c|c|c|c|}
\hline & \multicolumn{9}{|c|}{ QFac } \\
\hline & & 0.01 & 0.03 & 0.1 & 0.3 & 1 & 3 & 10 & 30 & 100 \\
\hline \multirow{9}{*}{ RFac } & 0.01 & 0.018 & 0.018 & 0.018 & 0.018 & 0.018 & 0.018 & 0.018 & 0.018 & 0.018 \\
\hline & 0.03 & 0.018 & 0.018 & 0.018 & 0.018 & 0.018 & 0.018 & 0.018 & 0.018 & 0.018 \\
\hline & 0.1 & 0.018 & 0.018 & 0.018 & 0.018 & 0.018 & 0.018 & 0.018 & 0.018 & 0.018 \\
\hline & 0.3 & 0.019 & 0.018 & 0.018 & 0.018 & 0.018 & 0.018 & 0.018 & 0.018 & 0.018 \\
\hline & 1 & 0.045 & 0.019 & 0.018 & 0.018 & 0.018 & 0.018 & 0.018 & 0.018 & 0.018 \\
\hline & 3 & 0.131 & 0.045 & 0.019 & 0.018 & 0.018 & 0.018 & 0.018 & 0.018 & 0.018 \\
\hline & 10 & 0.309 & 0.142 & 0.045 & 0.019 & 0.018 & 0.018 & 0.018 & 0.018 & 0.018 \\
\hline & 30 & 0.485 & 0.309 & 0.131 & 0.045 & 0.019 & 0.018 & 0.018 & 0.018 & 0.018 \\
\hline & 100 & 0.724 & 0.504 & 0.309 & 0.142 & 0.045 & 0.019 & 0.018 & 0.018 & 0.018 \\
\hline
\end{tabular}

Table 5.5 (Case 3) The $M A R E(Z)$ as $R F a c$ and $Q F a c$ are varied using IEKF

\begin{tabular}{|c|c|c|c|c|c|c|c|c|c|c|}
\hline & \multicolumn{9}{|c|}{ QFac } \\
\hline & & 0.01 & 0.03 & 0.1 & 0.3 & 1 & 3 & 10 & 30 & 100 \\
\hline \multirow{9}{*}{ RFac } & 0.01 & 0.015 & 0.015 & 0.015 & 0.015 & 0.015 & 0.015 & 0.015 & 0.015 & 0.015 \\
\hline & 0.03 & 0.015 & 0.015 & 0.015 & 0.015 & 0.015 & 0.015 & 0.015 & 0.015 & 0.015 \\
\hline & 0.1 & 0.015 & 0.015 & 0.015 & 0.015 & 0.015 & 0.015 & 0.015 & 0.015 & 0.015 \\
\hline & 0.3 & 0.015 & 0.015 & 0.015 & 0.015 & 0.015 & 0.015 & 0.015 & 0.015 & 0.015 \\
\hline & 1 & 0.017 & 0.015 & 0.015 & 0.015 & 0.015 & 0.015 & 0.015 & 0.015 & 0.015 \\
\hline & 3 & 0.023 & 0.017 & 0.015 & 0.015 & 0.015 & 0.015 & 0.015 & 0.015 & 0.015 \\
\hline & 10 & 0.043 & 0.024 & 0.017 & 0.015 & 0.015 & 0.015 & 0.015 & 0.015 & 0.015 \\
\hline & 30 & 0.069 & 0.043 & 0.023 & 0.017 & 0.015 & 0.015 & 0.015 & 0.015 & 0.015 \\
\hline & 100 & 0.099 & 0.072 & 0.043 & 0.024 & 0.017 & 0.015 & 0.015 & 0.015 & 0.015 \\
\hline
\end{tabular}

Table 5.6 (Case 3) The $M A R E(R)$ as $R F a c$ and $Q F a c$ are varied using IEKF 


\section{Case 4: Random changes in $Z$ with measurement step time varying}

The next investigation considers how the measurement step time $T$ affects the accuracy of tracking. The mean user think time $Z$, and thus the request arrival rate, changed at random instants as Eq.(5.8). $Z$ was chosen from a distribution with mean $1000 \mathrm{~ms}$ and $C_{Z}=1$ (thus, $\mathbf{Q}$ was set to $10^{6}$ ). The average time between changes is $40 T^{*}$. The relative measurement interval was varied over the range $\gamma_{T}=[0.4,20]$, so that the measurement errors were varied from $8 \%$ to $1.1 \%$ from Eq. (5.7). And parameter changing rate varied from one change per 100 measurement steps $(100 T)$ to one change per 2 measurement steps ( $2 T)$ by average.

Figure 5.15 and Figure 5.16 plot the MAREs and AMAREs with standard deviations (a $95 \%$ confidence interval is about twice as wide) of the tracking error of user think time $Z$ $(M A R E(Z))$ and prediction error of user response time $R(M A R E(R))$ from simulations with 10000 measurement steps. The horizontal axis gives $\log _{10} \gamma_{T}$, which ranges from -0.4 to 1.3. The values of MAREs are smaller than the values of fMAREs. The larger the $\gamma_{T,}$, the larger the difference between MARE and fMARE for tracking parameters. The large $\gamma_{T}$ indicates the large measurement step time which leads to fast change rate and big differences between consecutive measurement steps. It is less accurate to use the current estimate to represent the system status of the next step.

In Figure 5.15, the tracking error of $Z$ decreases when the measurement step size increases. The prediction error of $R$ is quite small and is not sensitive to $\gamma_{T}$. Basically larger measurement step size (smaller measurement errors) provides better tracking. In Figure 5.16, we see that there is an optimal step size around $2 T^{*}$ for parameter tracking. For performance prediction the optimum is around $4 T^{*}$, but the errors are rather insensitive. The parameter tracking error curve rises less steeply on the left, indicating that it is better to have measurement step size too small than too large for the purpose of prediction. The reason is that the filter averages the excessive measurement errors for small $T$, across successive steps, but it cannot compensate for falling behind the drift when $T$ is large. 


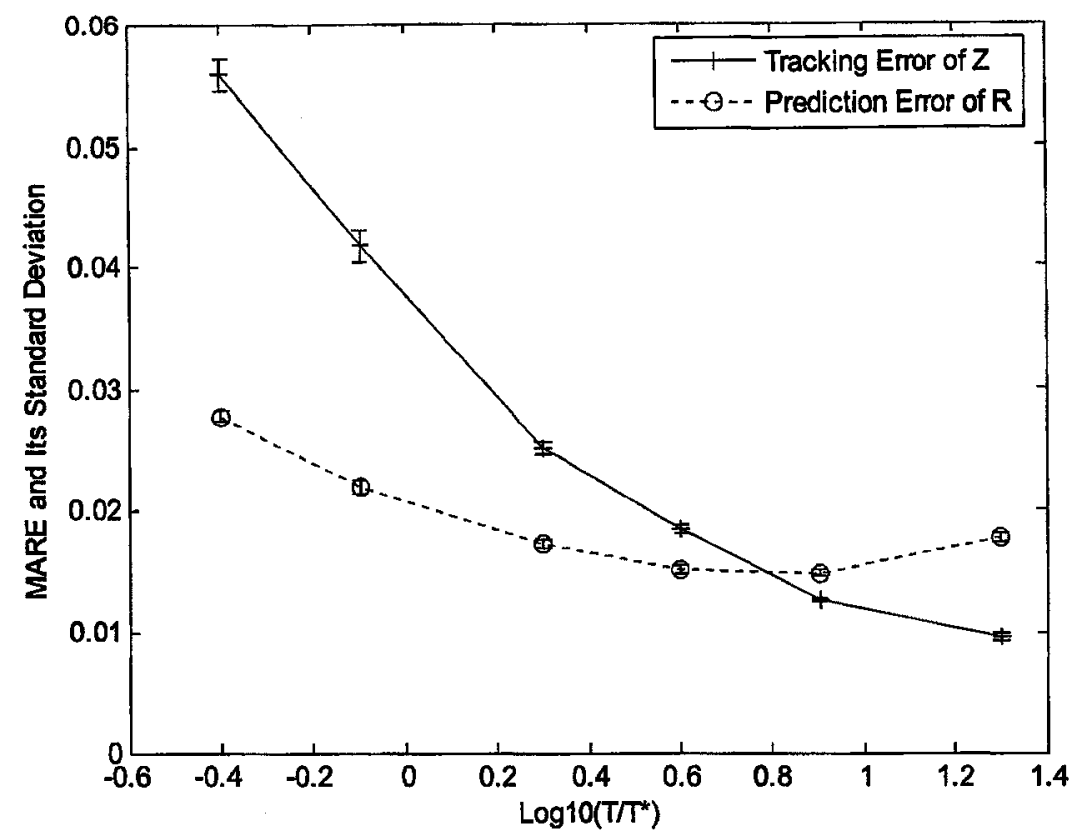

Figure 5.15 (Case 4) MAREs in Tracking Random Changes in User Think Time $Z$, for Different Lengths of the Measurement Interval $(T)$

From the trend shown in Figure 5.16, the measurement step time is important to the tracking quality when the filter is applied to make decisions. If the measurement step is too small, the measurement errors will be too big to have a good tracking quality. When the measurement step becomes large, the parameter changer per measurement step time increases, which leads to greater tracking errors. It seems to have an optimal value or region to balance the measurement error and change rate. 


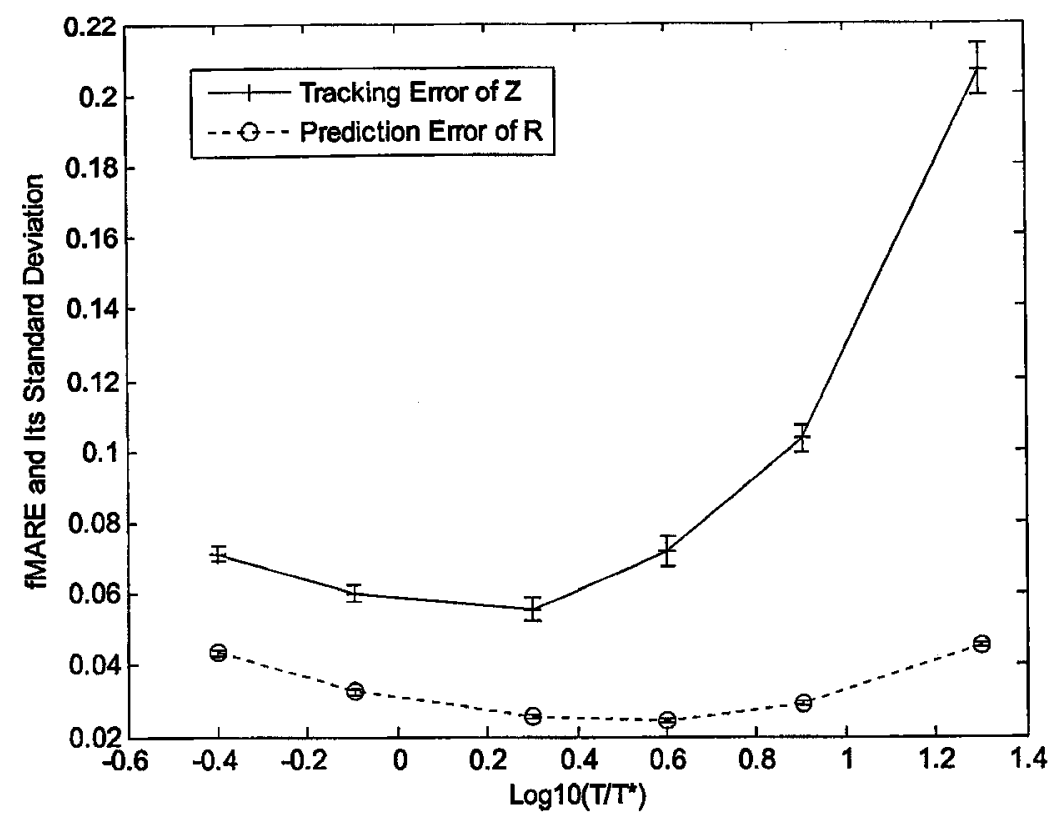

Figure 5.16 (Case 4) fMAREs in Tracking Random Changes in User Think Time $Z$, for Different Lengths of the Measurement Interval $(T)$

\section{Case 5: Random changes in $S_{d}$ with measurement step time varying}

Disturbances to service demands can arise when the users of a system swing to using different functions or using functions differently, for example to requiring larger database searches for each operation. They can also result from system aging, for instance due to memory leaks.

The mean database server demand $S_{d}$ changed randomly, using the same random disturbance process as for $Z$ in case 4 . The average value is $m_{S d}=10 \mathrm{~ms}$., and $C_{S d}=1$.

As in Case 4, the normalized measurement time interval was varied from $\gamma_{T}=0.4$ to 20, and the MAREs of $S_{d}$ and $R$ were determined. The total simulation measurement step was 10000 as well. 


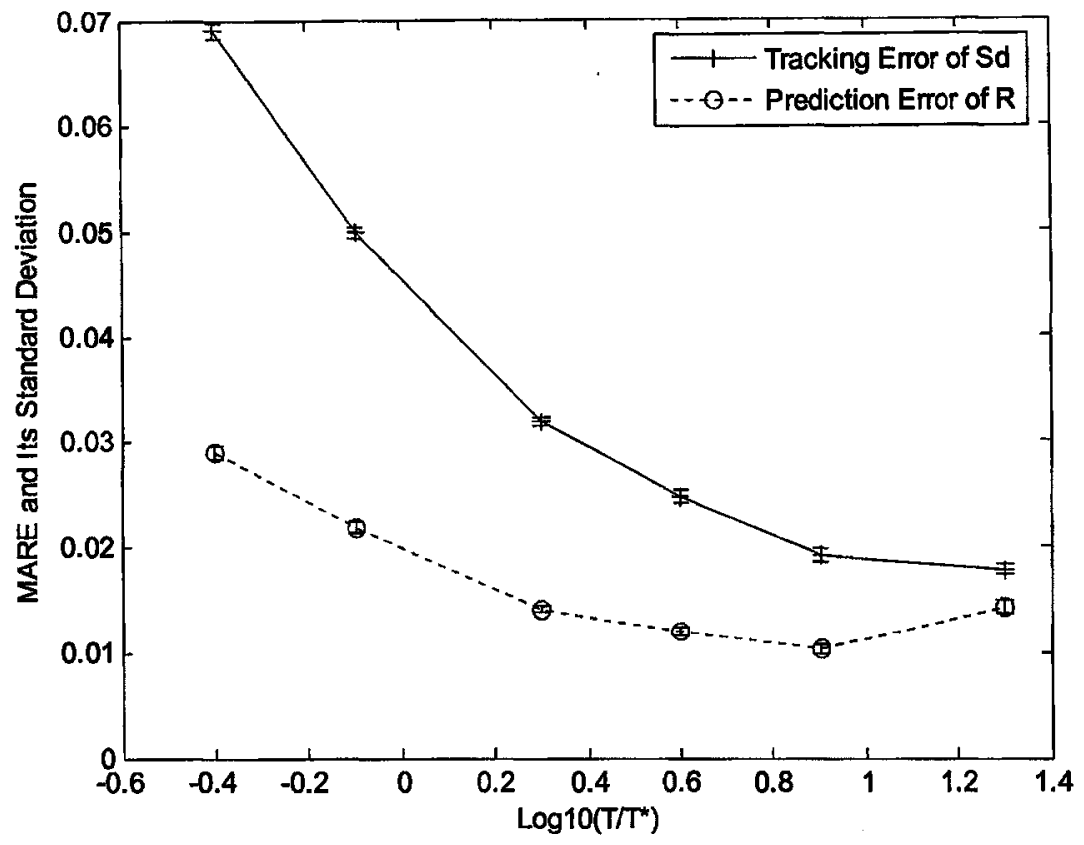

Figure 5.17 (Case 5) MAREs in Tracking Random Changes in $S_{d}$, for Different Lengths of the Measurement Interval ( $T$ )

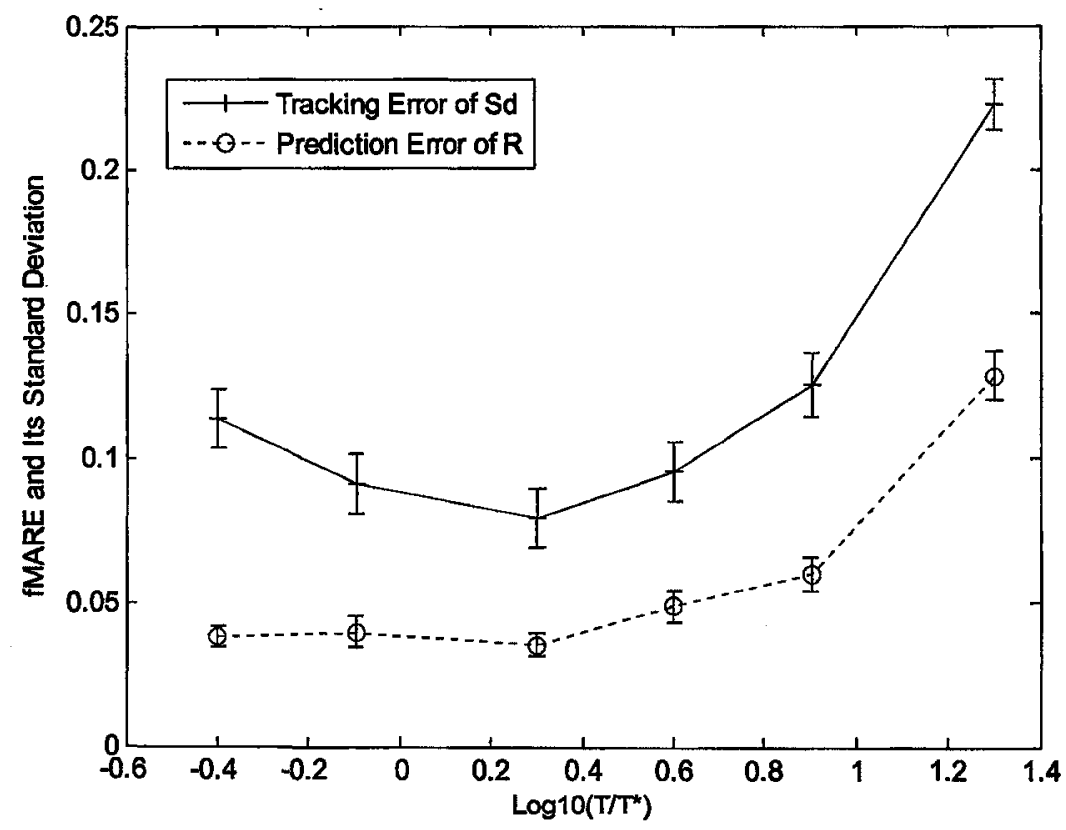

Figure 5.18 (Case 5) fMAREs in Tracking Random Changes in $S_{d,}$, for Different Lengths of the Measurement Interval $(T)$

The curves in Figure 5.17 and Figure 5.18 are similar to the curves in Figure 5.15 and 
Figure 5.16 respectively. The MAREs are smaller than AMAREs. Bigger $\gamma_{T}$ tends to make the difference between fMAREs and MAREs bigger. Generally the MAREs have smaller errors when the measurement step time is larger, while the fMAREs has a sharp slope after a threshold. The fMAREs have an optimal value which is around $T=2 T^{*}$.

\section{Case 6: Random changes in $Z$ with Different Disturbance Variances $C$ varying}

This case considers different distributions, variances of the random changes to the think time $Z$. The coefficient of variation $C_{Z}$ of the disturbances to $Z$ was varied over a range from 0.1 (quite small changes) to 2 . The other parameters took the base values. As expected, Figure 5.19 and Figure 5.20 show that the MAREs and AMAREs are small when the disturbance variance $C$ is small and the errors increase when $C$ becomes large. Big coefficient of variation implies big value change which results in large errors. The fMAREs in Figure 5.20 are bigger than the MAREs in Figure 5.19. The difference is more significant when $C$ is big. All the MAREs are very small and fMAREs are no more than $10 \%$.

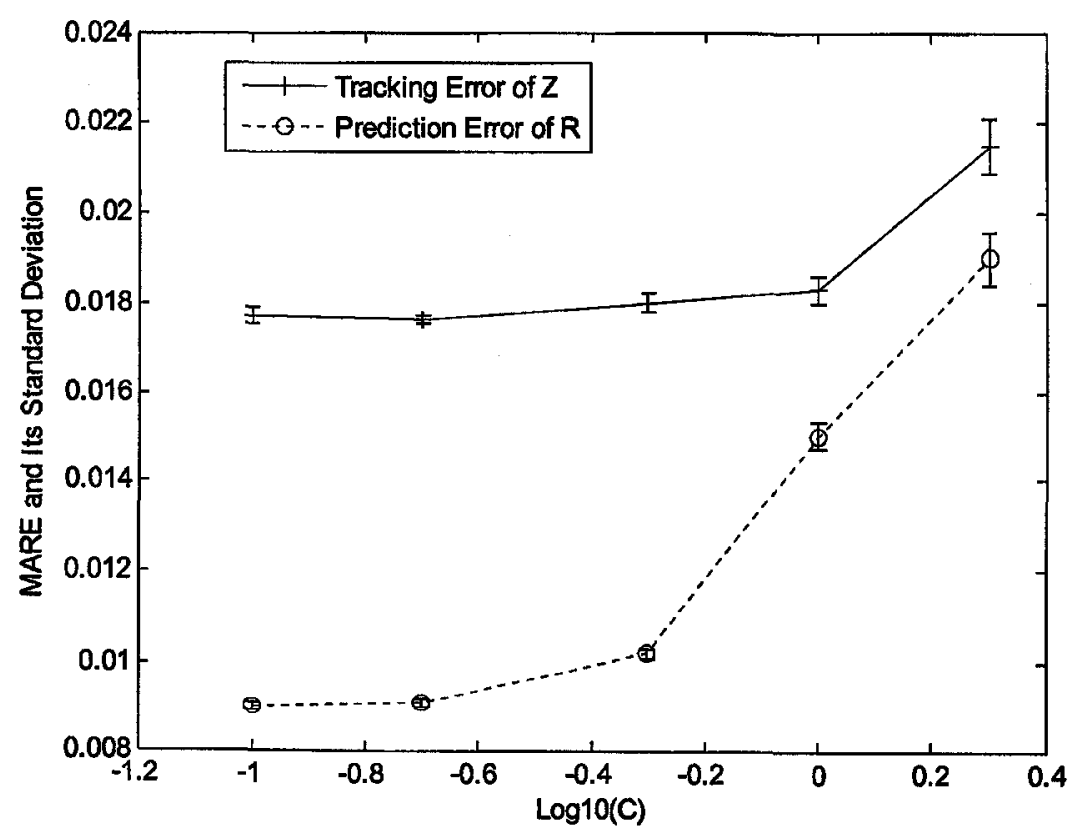

Figure 5.19 (Case 6) MAREs in Tracking Random Changes in User Think Time $Z$, for Different Values of $\boldsymbol{C}$ 


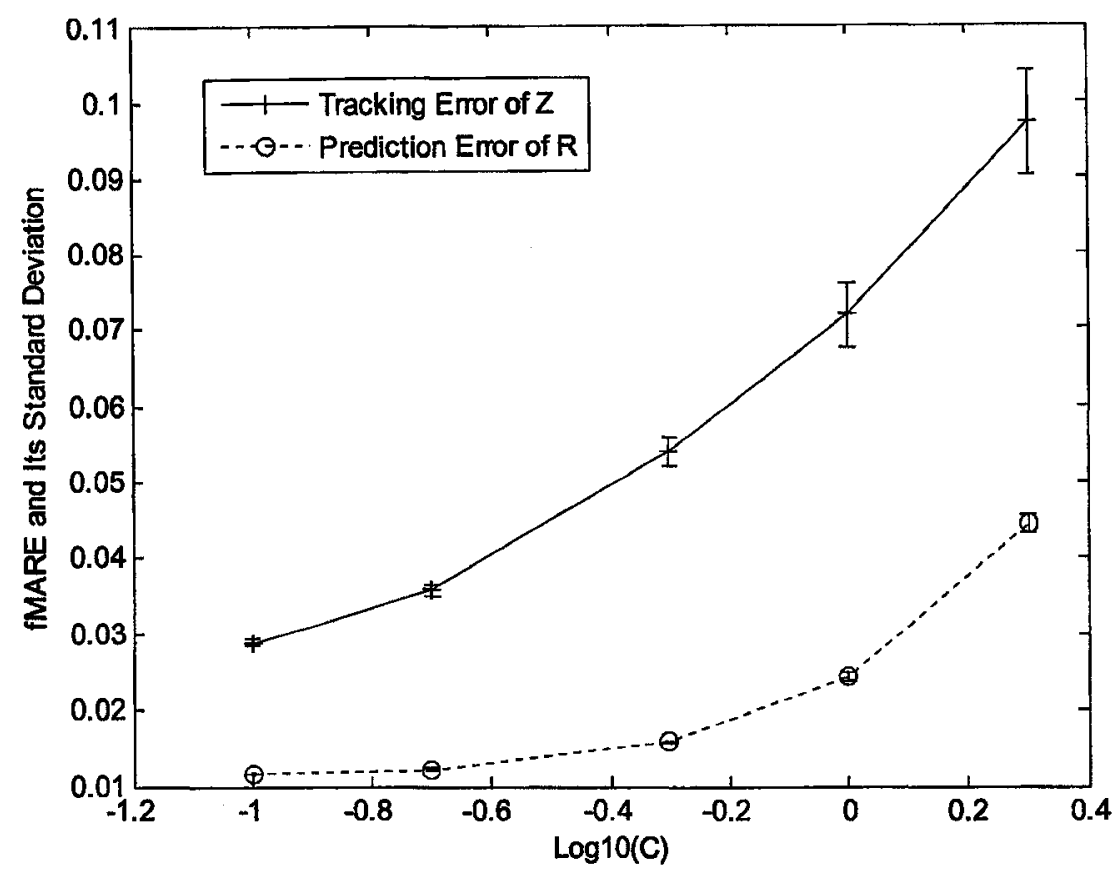

Figure 5.20 (Case 6) fMAREs in Tracking Random Changes in User Think Time $Z$, for Different Values of $C$

\section{Case 7: Random changes in $S_{d}$ with Different Disturbance Variances $C$ varying}

In this case, different variances of the random changes are applied to the database demand $S_{d}$. The coefficient of variation $C_{S d}$ of the disturbances to $S_{d}$ was varied over a range from 0.1 to 2 . The other parameters took the base values. Figure 5.21 and Figure 5.22 show how the MAREs and AMAREs change when $C_{S d}$ changes. The trends of the curves in those two figures are similar to the curves in Figure 5.19 and Figure 5.20 that the errors increase when $C$ increases. In Figure 5.22, the fMAREs are less than $10 \%$ if $C$ is no more than 1 .

There is something common in case 4 - case 7. All the MAREs are less than 0.1 . Most of the time they are less than 0.05 . It indicates that it is quite good to estimate system parameters using IEKF. The fMAREs are always larger than MAREs as fMAREs compare the current estimation with the measurement of the next step instead of the current measurement in MAREs. One aim of the tracking filter is to guide the decision making, so $\mathrm{AMARE}$ is more informative for this purpose. In the rest of the chapter, only fMARE will be used to measure the tracking quality. 


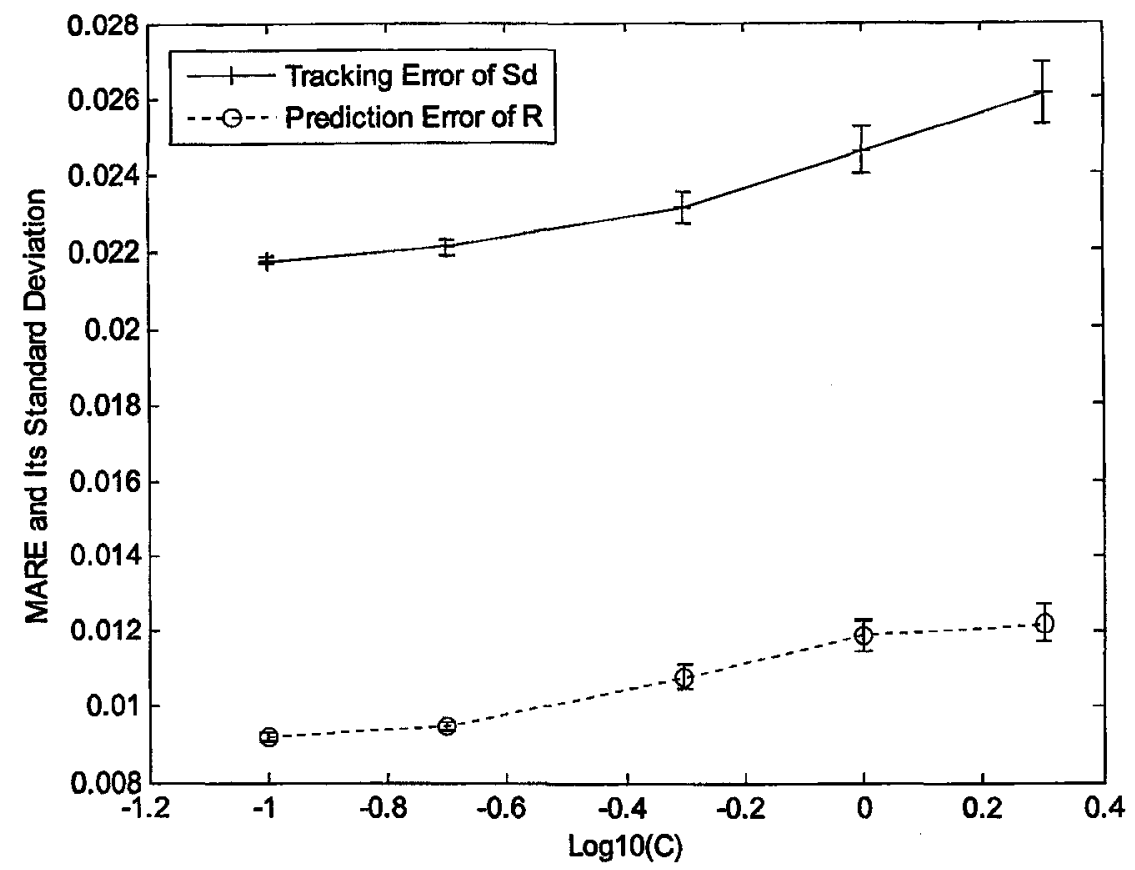

Figure 5.21 (Case 7) MAREs in Tracking Random Changes in $S_{d}$, for Different Values of $\boldsymbol{C}$

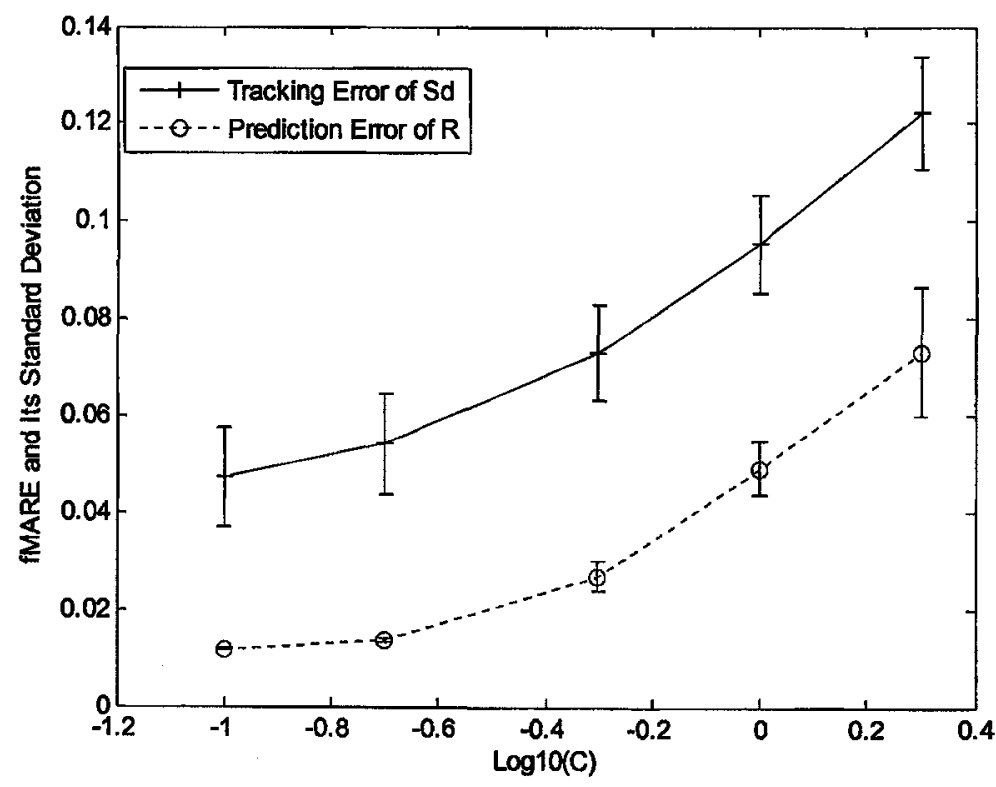

Figure 5.22 (Case 7) MAREs in Tracking Random Changes in $S_{d}$, for Different Values of $C$ 


\subsection{Tracking three parameters}

In the previous cases, only one parameter was changed. In this section, three parameters might be changed independently. As in real systems, it is more likely that different system parameters may change independently. In this case, the parameters that will change are the user think time $Z$ (at a mean value of $1000 \mathrm{~ms}$ ), the Database demand $S_{d}$ (at a mean value of $10 \mathrm{~ms}$ ) and the $W e b S e r v e r$ demand $S_{w}$ (at a mean value of $5 \mathrm{~ms}$ ). The new values of those parameters are generated by Eq. (5.8) as used in case 4 - case 7. Two sets of measurement vectors will be used to evaluate how the measurement set affects the tracking quality. The first measurement set is

$\mathbf{z 1}=\left[R, U_{w}, U_{b}, U_{d}\right]$ which is the same as the one used in the previous sections. The second measurement set is $\mathbf{z 2}=\left[R, U_{w}, U_{b}, U_{d b} \lambda, R_{w}, R_{d}\right]$

where

$\lambda$ is the throughput of the user,

$R_{w}$ is the response time of the web sever

$R_{d}$ is the response time of the database

The metrics to measure the tracking quality are $f M A R E(Z), f M A R E\left(S_{d}\right), f M A R E\left(S_{w}\right)$ and $f M A R E(R) . f M A R E(Z)$ is the tracking error of the user think time $Z, f M A R E\left(S_{d}\right)$ is the tracking error of Database demand $S_{d,}$, $M A R E\left(S_{w}\right)$ is the tracking error of WebServer demand $S_{w}$ and $f M A R E(R)$ is the prediction error of the user response time $R$.

\section{Case 8: Measurement Time with three parameters changing}

Figure 5.23 and Figure 5.24 show the PMAREs with $\gamma_{T}$ ranging from 0.4 to 20 using IEKF with 2 sets of measurement vector. $f M A R E(R)$ varied from 0.065 (when $\gamma_{T}=0.4$ ) to 0.40 (when $\gamma_{T}=20$ ). When $\gamma_{T}$ is no more than $4, f M A R E(R)$ is no more than 0.15 . However, some of the tracking errors are very big especially $f M A R E(Z)$ in Figure 5.24 when $\gamma_{T}=8$. After examining the tracking logs, it was found that the big errors mainly came from a small number of steps when all the three tracking parameters have big step changes simultaneously. During those measurement steps, the user response time $R$ was 50 times bigger than the default settings. The estimated parameters were far away from the real values for a number of measurement steps. This occurrence is unlikely, and is 
well outside the assumed "usual" behaviour, for which the filter was tuned.

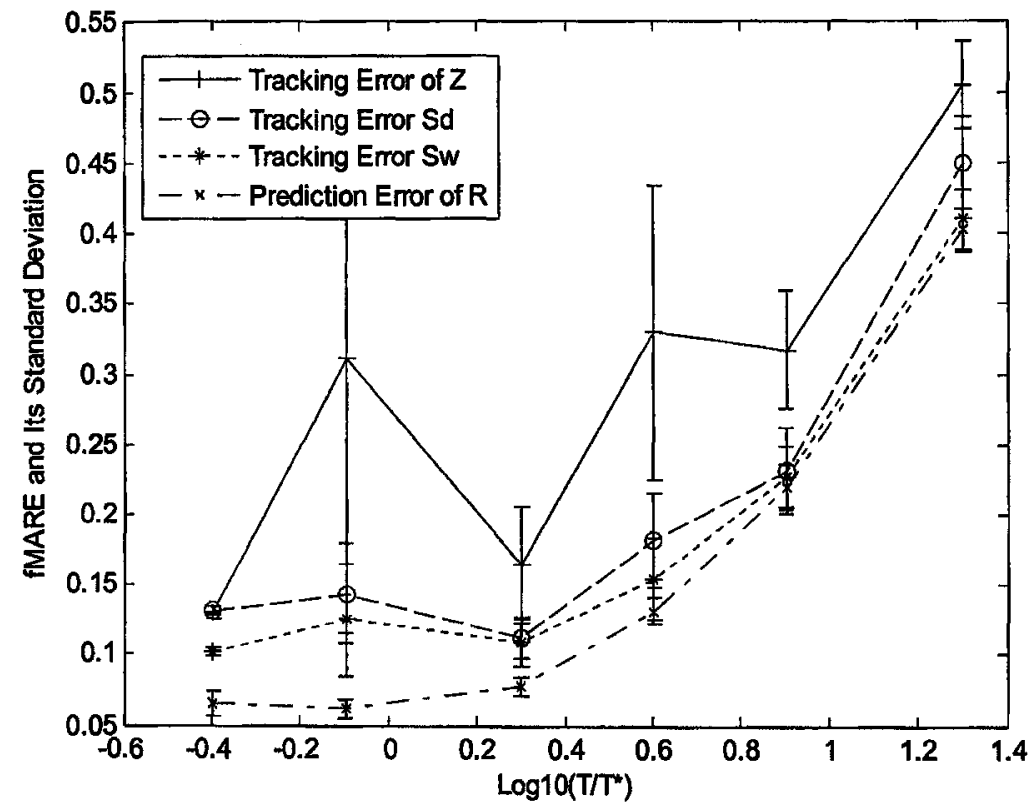

Figure 5.23 (Case 8) fMAREs as $T$ is varied for three parameters changing (4 measurements with fixed $R$ values)

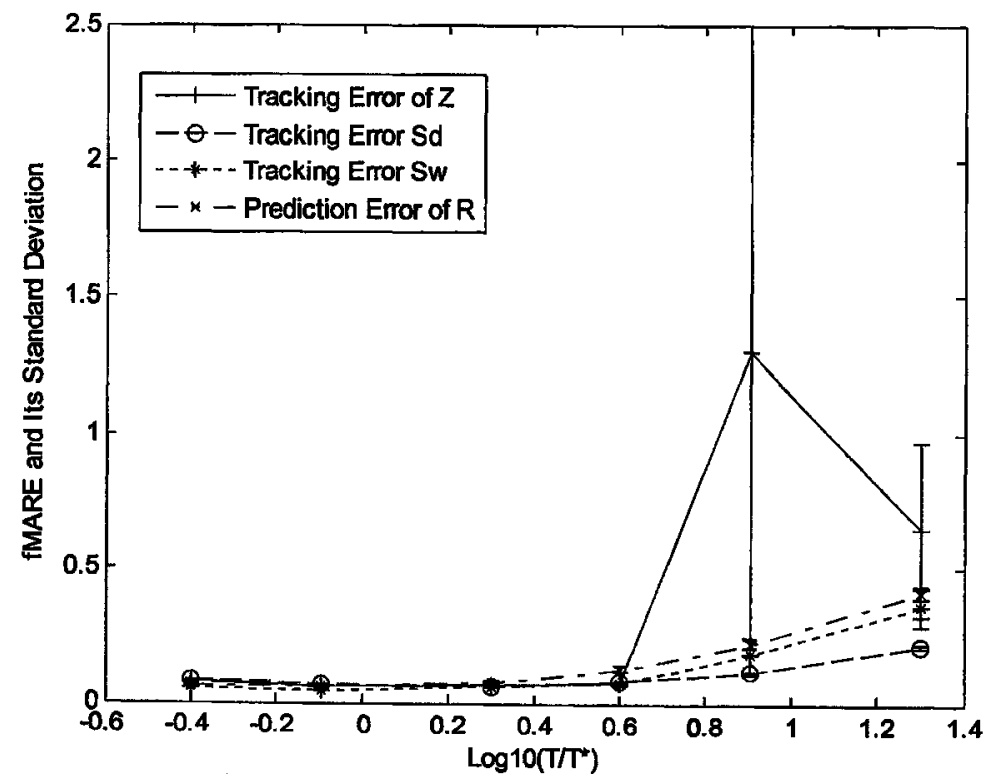

Figure 5.24 (Case 8) fMAREs as $T$ is varied for three parameters changing (7 measurements with fixed $R$ values) 


\subsection{Tracking with Adaptive $R$}

In order to get better tracking quality with simultaneous large jump in the system behavior, two adaptive $\mathbf{R}$ approaches were implemented and evaluated.

The first approach was introduced in [35]. The $\mathbf{R}$ values are calculated from the history data using statistics.

$$
\begin{aligned}
& \hat{R}=\frac{1}{N-1} \sum_{i=1}^{N}\left(v_{i}-\bar{v}\right)\left(v_{i}-\bar{v}\right)^{T} \\
& \bar{v}=\frac{1}{N} \sum_{i=1}^{N} v_{i}
\end{aligned}
$$

where $v_{i}$ is the measurement error at sample $i$ and $\bar{v}$ is the mean value of all the $v_{i}$. Eq. (5.11) and Eq. (5.12) requires all the history of $v_{i}$ to calculate $\bar{v}$. It is not practical to implement in real systems. An iterative representation assigning different weight to the last previous value and history values is shown in Eq. (5.13) and Eq. (5.14):

$$
\begin{aligned}
& \hat{R}_{k+1, i i}=(1-\alpha) \hat{R}_{k, i i}+\alpha\left(e_{k+1, i}-\hat{e}_{k+1, i}\right)^{2} \\
& \hat{e}_{k+1, i}=(1-\alpha) \hat{e}_{k, i}+\alpha e_{k+1, i}
\end{aligned}
$$

where $\hat{R}_{k+1, i}$ is the $i$ th diagonal value of $\mathbf{R}$ at step $k+1, e_{k+1, i}$ is the measurement error of the $i$ th measurements at step $\mathrm{k}+1$ and $\hat{e}_{k+1, i}$ is the estimated average measurement error of the $i$ th measurements. The value of $\alpha$ was set to 0.1 . In this case, $\alpha$ was arbitrary and more evaluation is possible.

Figure 5.25 and Figure 5.26 shows the fMAREs of the adaptive $\mathbf{R}$ approach using Eq. (5.13) and Eq. (5.14). By comparing them with Figure 5.23 and Figure 5.24, the tracking quality was found even worse. This adaptive $\mathbf{R}$ approach used curve fitting to estimate the values in $\mathbf{R}$. It provides good estimation if the systems stays around a specific value. However, in dynamic systems, the system parameters change frequently and the change amplitude might be big. The curve fitting or moving average method may not represent the current measurement error well. 


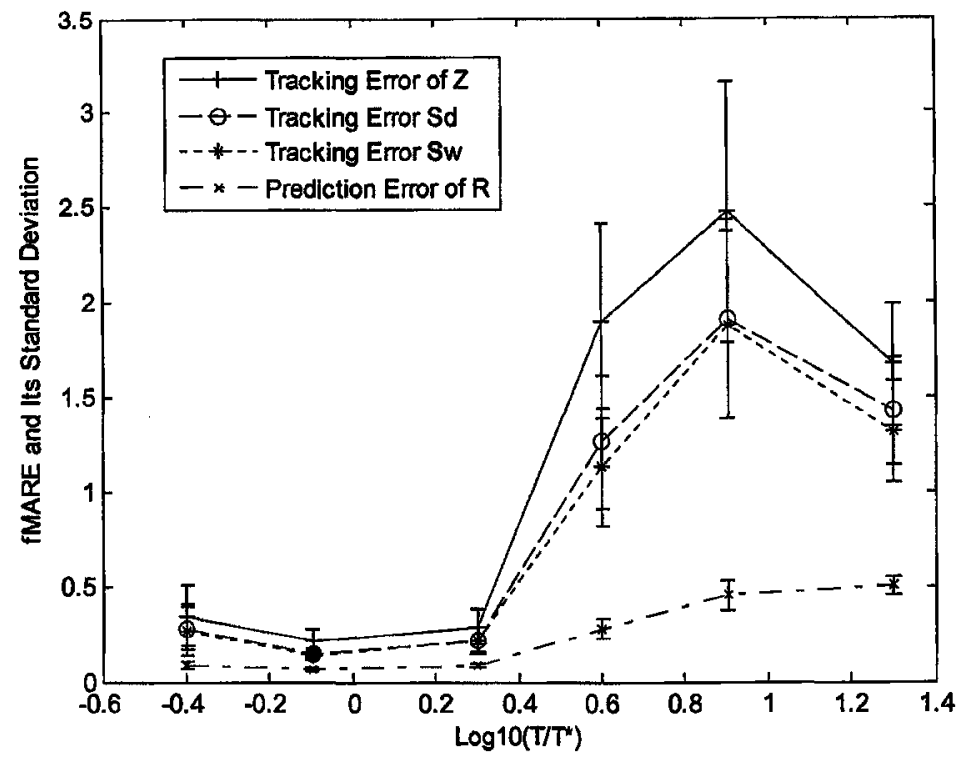

Figure 5.25 (Case 8) fMAREs as $T$ is varied for three parameters changing (4 measurements with adaptive $R$ values from Eq. (5.13) and Eq. (5.14))

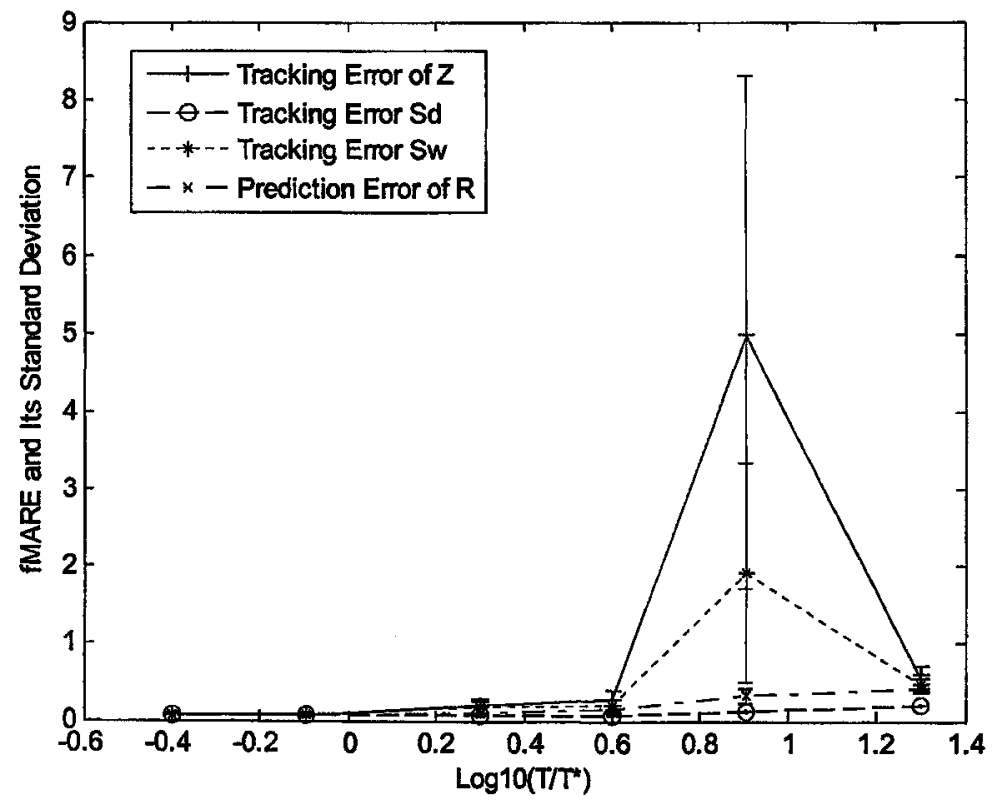

Figure 5.26 (Case 8) fMAREs as $T$ is varied for three parameters changing (7 measurements with adaptive $R$ values from Eq. (5.13) and Eq. (5.14))

The second adaptive $\mathbf{R}$ approach is a heuristic one which uses Eq.(5.15) to replace Eq.(5.7) in fixed $\mathbf{R}$ approach 


$$
R_{k, i i}=\left(0.0255 z_{k, i}\right)^{2} / \gamma_{T}
$$

In Eq. (5.15), the diagonal value of $\mathbf{R}$ matrix at step $k$ depends on the related measurements at step $k$. The measurement error covariance is assumed proportional to square of the real measured values. The fMAREs in Figure 5.27 and Figure 5.28 are smoother and smaller than the values in Figure 5.23 - Figure 5.26. The adaptive $\mathbf{R}$ approach using Eq.(5.15) was quite satisfactory. In the later part of this chapter, this adaptive $\mathbf{R}$ approach will be used to calculate values in $\mathbf{R}$.

The errors in Figure 5.27 (using 4 measurements) are generally larger than those in Figure 5.28 (using 7 measurements). It shows that the measurement vector with more measurements does help the tracking quality. With three parameters changing, the prediction error increases with a faster pace when $\gamma_{T}$ is more than 2 . The optimal $\gamma_{T}$ seems around $2 T^{*}$. When $\gamma_{T}$ is no more than $4 T^{*}$, the fMAREs are less than 0.15

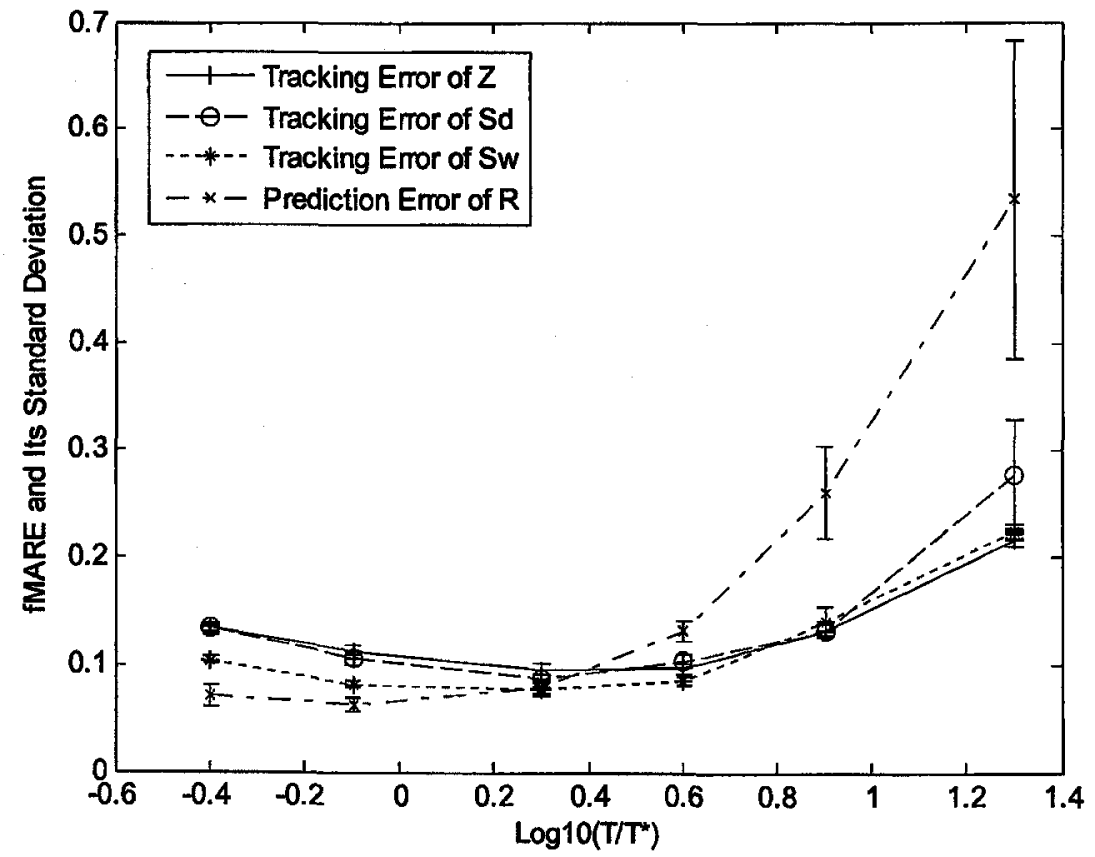

Figure 5.27 (Case 8) fMAREs as $T$ is varied for three parameters changing (4 measurements with adaptive $R$ values from Eq. (5.15)) 


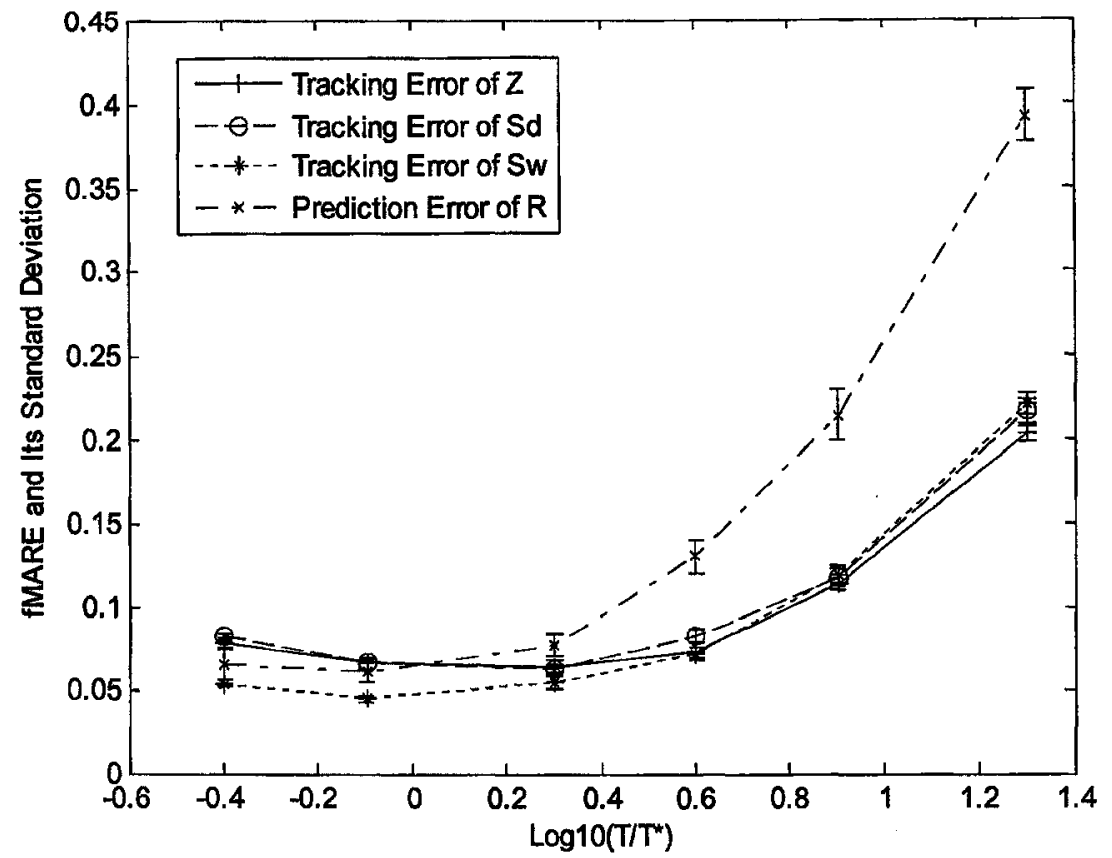

Figure 5.28 (Case 8) fMAREs as $T$ is varied for three parameters changing (7 measurements with adaptive $R$ values from Eq. (5.15))

\section{Case 9: Different Disturbance Variances $C$ with three parameters changing}

Figure 5.29 and Figure 5.30 show the AMAREs with $C$ ranging from 0.1 to 2 using IEKF with different sets of measurement vector. When disturbance increases, the tracking errors increase as expected. Similar to case 8 , the prediction error of the user response time increase in a fast pace when the disturbance increases. When $C$ is no more than 1, the fMAREs are under 0.15 from those two figures. The optimal of $\gamma_{T}$ is around $2 T^{*}$ in this case. 


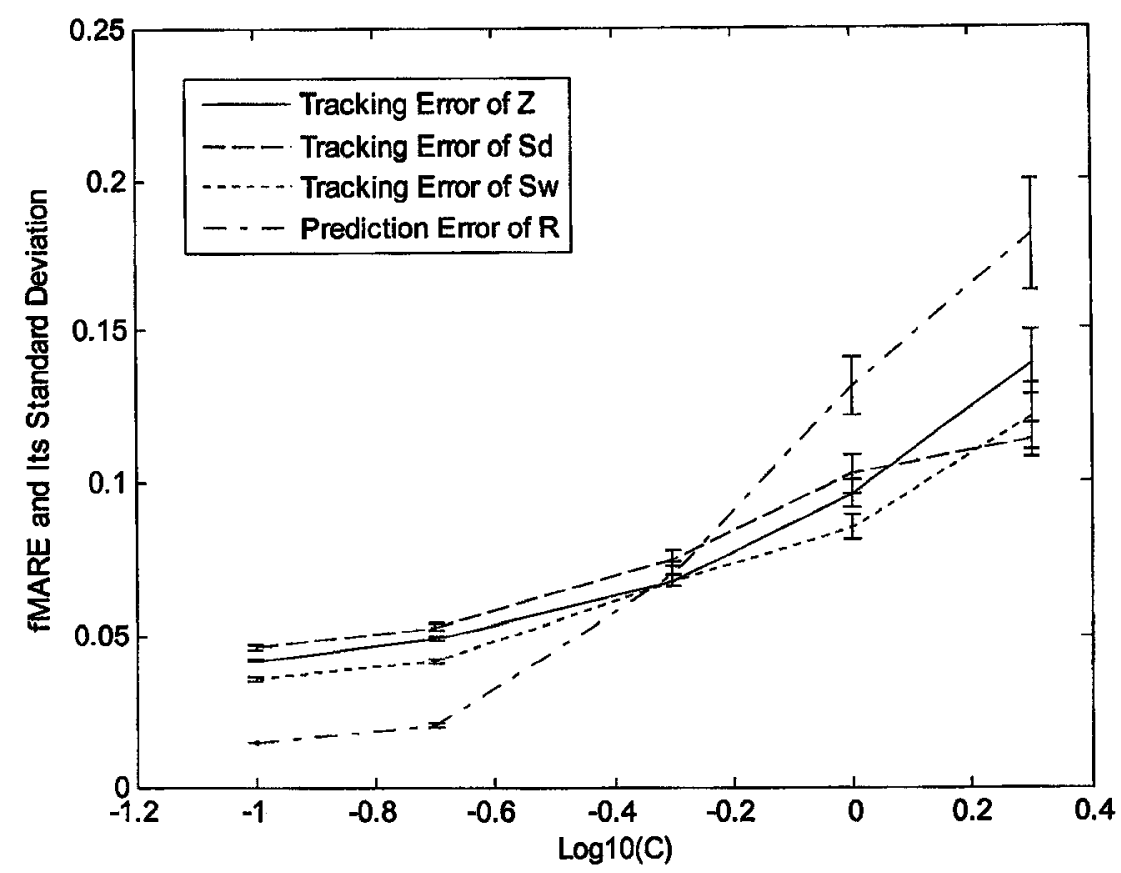

Figure 5.29 (Case 9) fMAREs as $C$ is varied for three parameters changing (4 measurements)

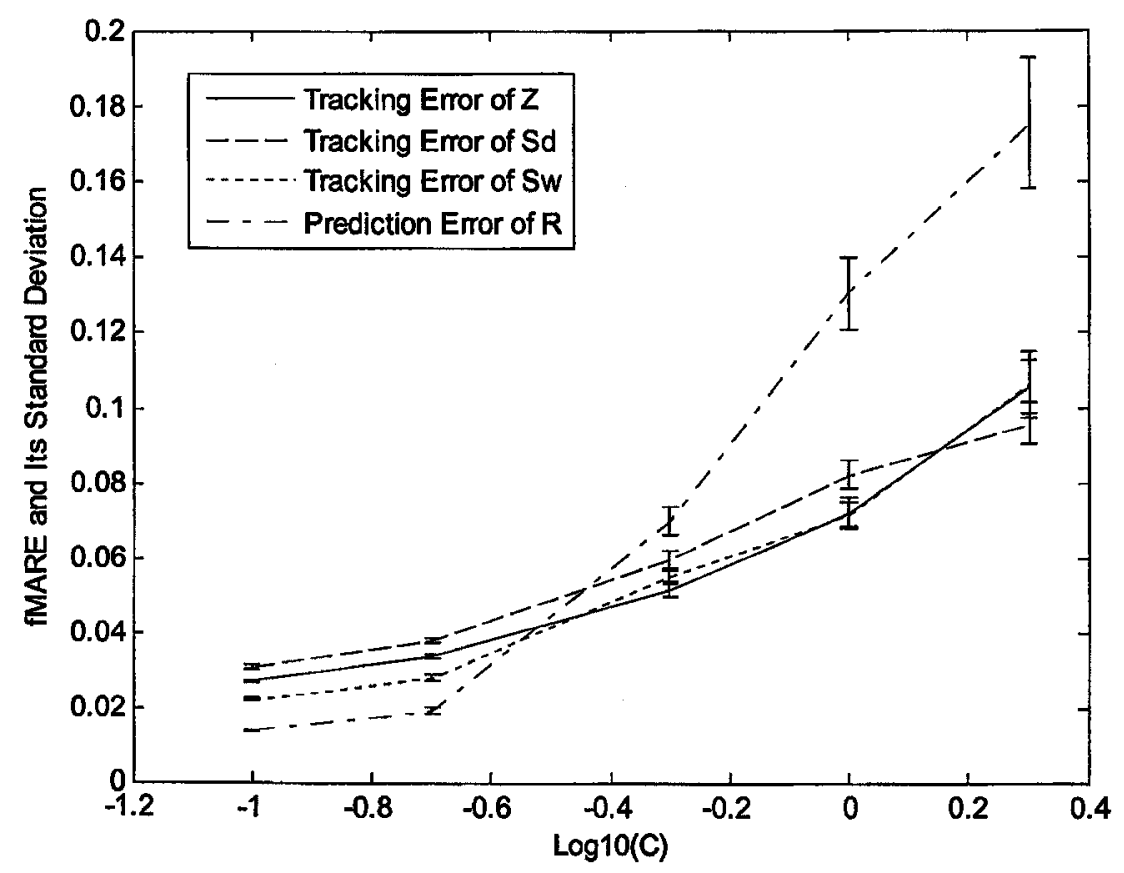

Figure 5.30 (Case 9) fMAREs as $C$ is varied for three parameters changing (7 measurements)

63 


\subsection{Evaluations on a model with two user classes}

In the previous model shown in Figure 2.2, there is only 1 class of users. Each task has only 1 entry. In this section, a model with two user classes (shown in Figure 5.31) will be evaluated. This model has 2 different classes of users with different numbers of users and user think times. The default values of users $N_{1}$ and $N_{2}$ are: $N_{1}=20$ and $N_{2}=10$. Each class of users has different probabilities for different operations. Furthermore, each task may have more than one entry, and the model has more layers than the previous one.

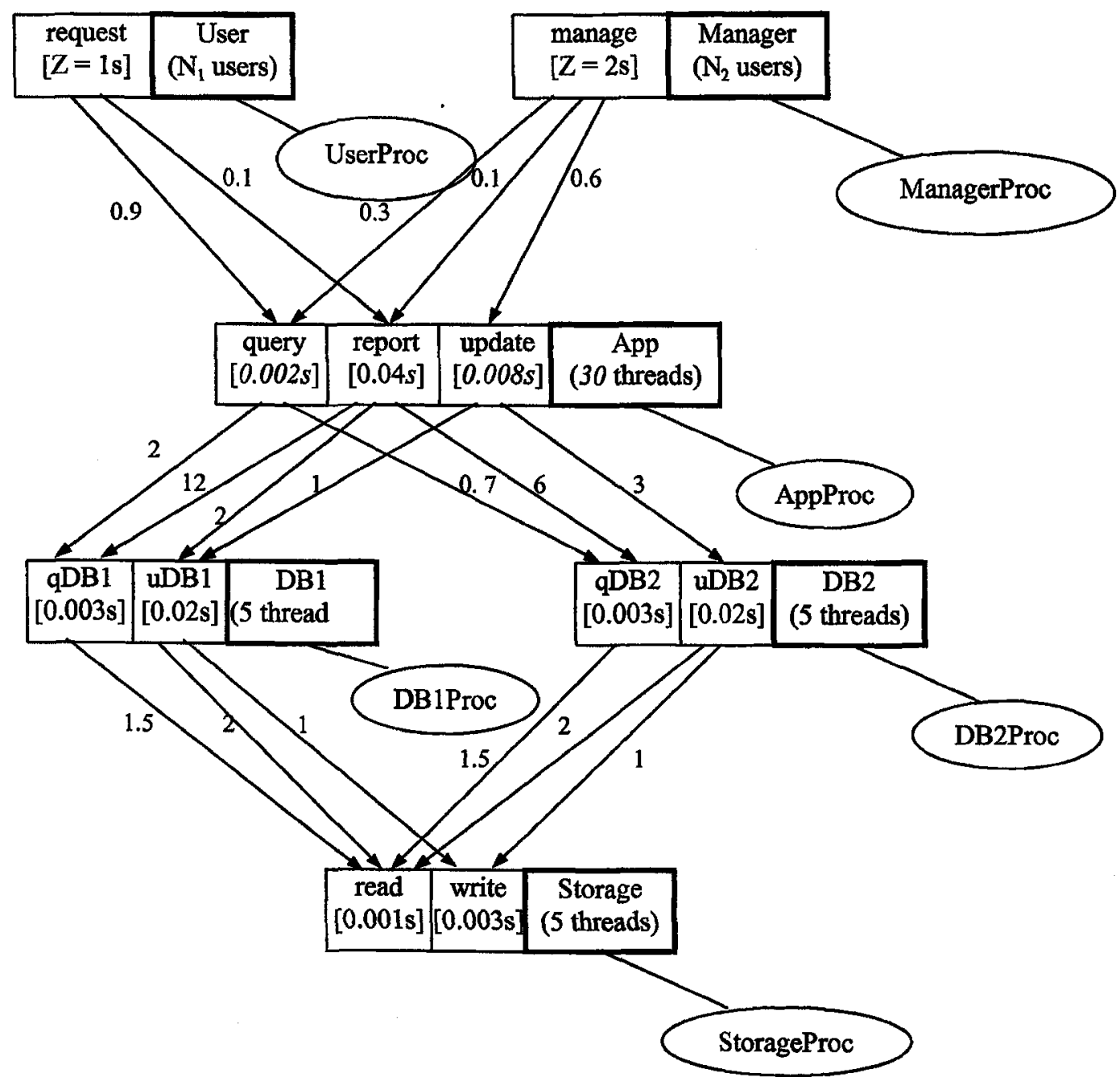

Figure 5.31 An LQN model with 2 user classes

The tracking parameter set $\mathbf{x}=\left[Z_{\text {user }}, Z_{\text {manager }} D_{\text {query }}, D_{\text {repory }}, D_{\text {update }}, D_{q D B 1}, D_{u D B 1}, D_{q D B 2}\right.$ $\left.D_{u D B 2}, D_{\text {read }} D_{\text {write }}\right]$ which includes the 2 user think times, 8 service demands of the entries. The measurements set $\mathrm{z}=\left[R_{\text {user }} R_{\text {manager }}, \lambda_{\text {user }}, \lambda_{\text {manager }} R_{\text {query }} R_{\text {reporr }} R_{\text {update }}, R_{u D B 1}, R_{u D B}\right.$, 64 
$\left.R_{q D B 2}, R_{u D B 2}, R_{\text {read }}, R_{\text {write, }}, U_{A p p,} U_{D B 1}, U_{D B 2,} U_{\text {storage }}\right]$ where $R$ represents the response times of different entries. $\lambda_{\text {tser }}$ and $\lambda_{\text {manager }}$ are the throughputs of 2 classes of users and $U_{A p p} U_{D B I}$, $U_{D B 2}, U_{\text {storage }}$ are the CPU utilizations of the processors. The standard measurement step size was set as $T^{*}=1900$ s to achieve the $95 \%$ confidence interval of the user response times no more than $5 \%$ of the mean values. The change pattern of the service demands was different from the one in the previous model. Each service demand has a probability of 0.1 to change at each $T^{*}$. Amplitude of each change is $5 \%$ of the default value. It could be either an increasement or decreasement with the probability of $50 \%$. The maximum amplitude is limited to $50 \%$ of the default value.

Figure 5.32 shows the PMAREs when measurement step time $T$ ranged from $0.01 \mathrm{~T}^{*}$ to $3 T^{*}$ (i. e. $\gamma_{T}$ ranged from 0.01 to 3 ). The $\log 10\left(\gamma_{T}\right)$ varied from -2 to 0.48 respectively. Most fMAREs of the tracking parameters were no more than 0.2 . They were no more than 0.1 when $\gamma_{T}$ was between 0.1 and 1 . However there are 2 tracking errors $f M A R E\left(D_{\text {update }}\right)$ and $f M A R E\left(D_{\text {queny }}\right)$ which were much bigger than the others. It was found that the entry "update" and "query" belong to the same task. Comparing them with the other entry "report" in the same task, $D_{\text {update }}$ and $D_{\text {query }}$ were much smaller than $D_{\text {report }}$. A 0.05 tracking error in $D_{\text {report }}$ (i.e. a tracking error of 0.002 ) is 0.25 for $D_{\text {query }}$ and 1.0 for $D_{\text {update. }} D_{\text {update }}$ and $D_{\text {query }}$ were less sensitive to the response times comparing with other tracking parameters. From the tracking traces, it was also found that when $D_{\text {update }}$ was larger than the actual value, $D_{\text {report }}$ was always smaller than the actual value. Each one compensated the errors of the other. In Figure 5.33, The prediction errors of the response times $\left(f M A R E\left(R_{\text {user }}\right)\right.$ and $\left.f M A R E\left(R_{\text {manager }}\right)\right)$ were no more than 0.05 if $\gamma_{T}$ is no less than 0.1 . 


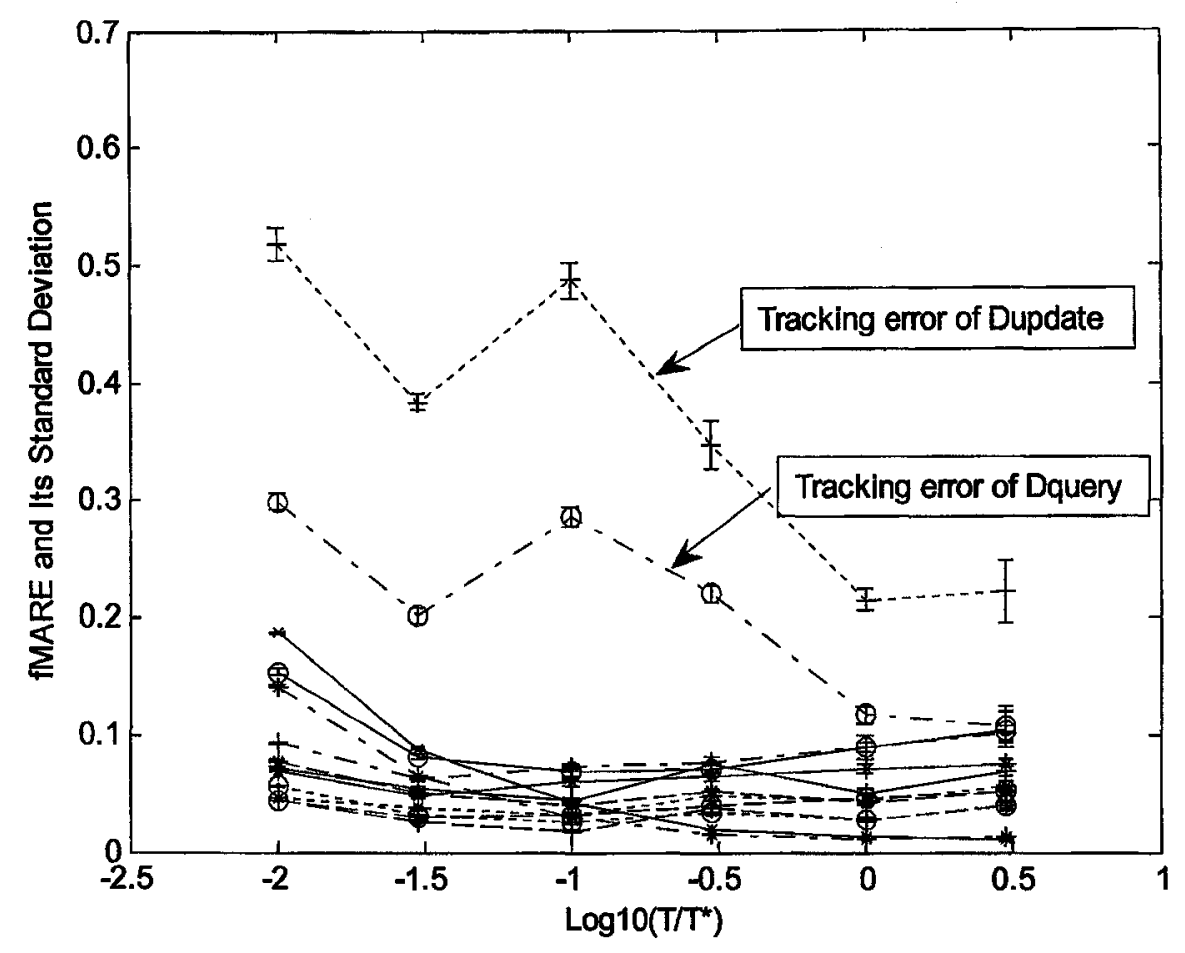

Figure 5.32 fMAREs as $T$ is varied for the model with 2 user classes

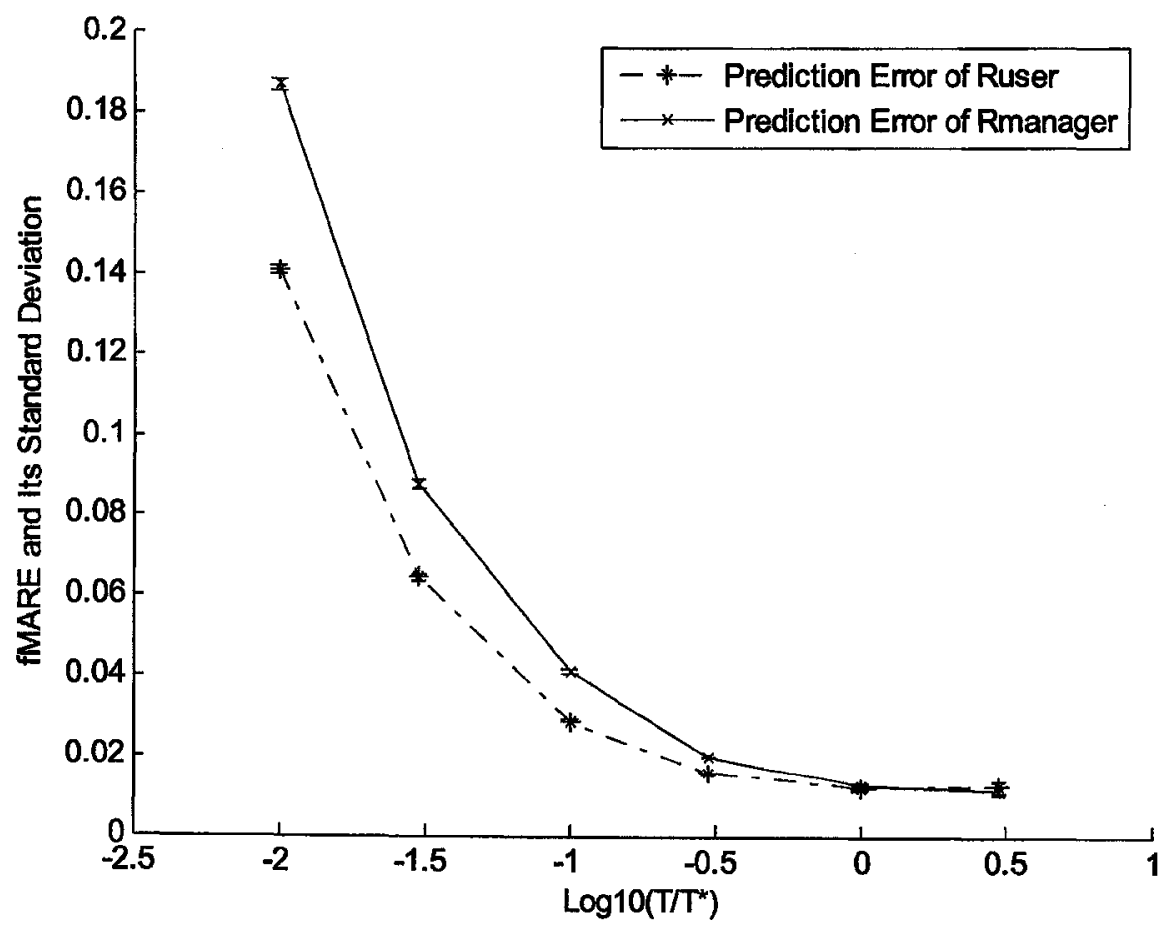

Figure 5.33 fMAREs as $T$ is varied for the model with 2 user classes 
The tracking errors $f M A R E\left(D_{\text {update }}\right)$ and $f M A R E\left(D_{\text {query }}\right)$ could be smaller if the CPU utilizations of all entries were available. Figure 5.34 shows that all the tracking errors were under 0.2 if the measurement set included the CPU utilization of each entry instead of the CPU utilization of each processor.

The tracking quality could be improved as well if service demands in task App are more balanced. Figure 5.35 shows the fMAREs when $D_{\text {update }}=0.02$ and $D_{\text {query }}=0.01$ instead of the previous values 0.002 and 0.008 respectively.

By comparing Figure 5.34 and Figure 5.35 with Figure 5.32 and Figure 5.33, the performance prediction errors $f M A R E\left(R_{\text {user }}\right)$ and $f M A R E\left(R_{\text {manager }}\right)$ were at the same level while the parameter tracking errors $f M A R E\left(D_{\text {update }}\right)$ and $f M A R E\left(D_{\text {query }}\right)$. It suggested that the more detailed measurements (CPU utilization of each entry vs. CPU utilization of each processor) helps the tracking quality.



Figure 5.34 fMAREs as $T$ is varied for the model with 2 user classes (having entry CPU utilization instead of processor CPU utilization) 


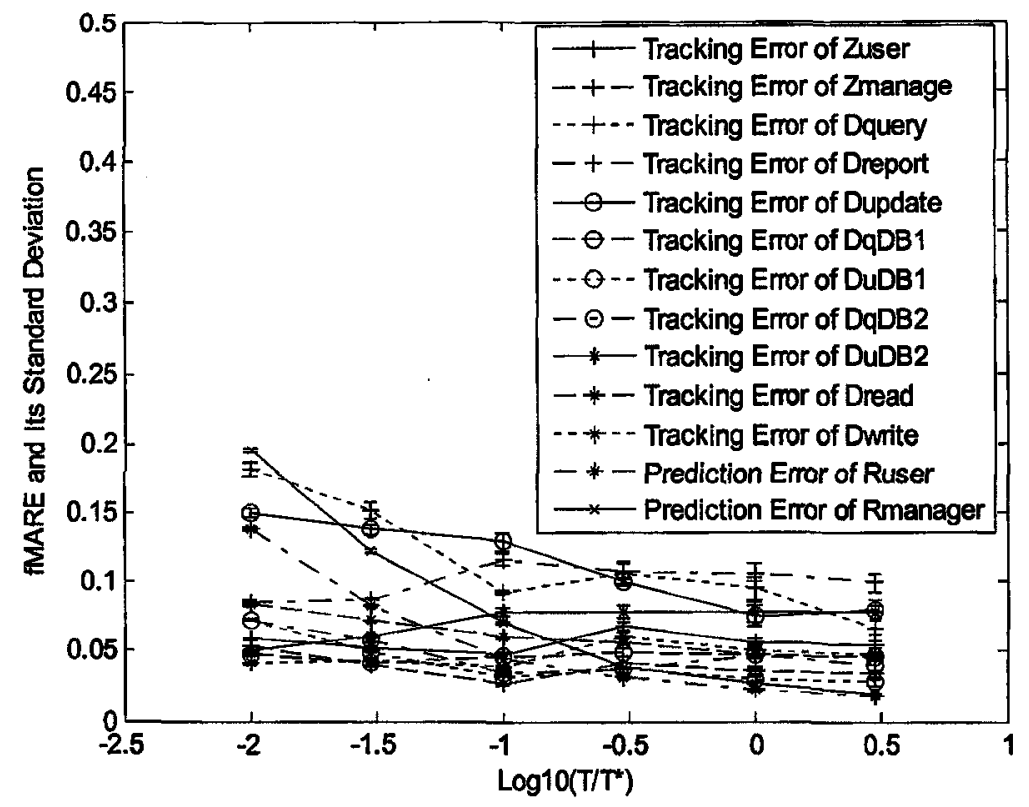

Figure 5.35 fMAREs as $T$ is varied for the model with 2 user classes $\left(D_{\text {update }}=0.02\right.$ and $\left.D_{\text {query }}=0.01\right)$ 


\subsection{Guidelines for Practical Implementation}

The iterated extended Kalman filter (IEKF) has showed better tracking quality than the EKF in both deterministic change and random change cases. This agrees with the conclusion in [25], that IEKF is the most informative tracking filter for systems with linear process model and nonlinear measurement model. It is a good choice to use IEKF to track multi tier dynamic system.

One of the most important issues in implementing the filter is the choice of the measurement step time $T . T$ is not only going to affect the performance of the filter, and hence the accuracy of the layered queuing model, but also it will dictate the timing for the actions of the Decision Maker block (see Figure 1.2). Regarding the latter relationship, in classical control, the control interval and measurement step time are the same: if the Decision Maker block actuates the system every second, the decision is based on the measurements taken in a second-long time window. The experiments showed that the performance of the filter is dependent on the measurement step time. The large value may decrease the measurement errors which is good to the tracking filter. However, if the measurement step time is so large that the parameters change rate is fast relative to the measurement step time, it may deteriorate the tracking quality. It is better to have some knowledge of the change rate of the system to choose a desirable measurement step time which balances the measurement errors and change rate. From the experiment results, it seems $0.1 T^{*}-T^{*}$ is a good interval for measurement step size if the change period is not shorter than $10 T^{*}$. The fMAREs of tracking parameters and predicted user response times are under 0.15 if variance of the change amplitude $C$ is no more than 1.

Other parameters needed for the implementation of the filter are the covariance matrices $\mathbf{Q}$ and $\mathbf{R}$ that characterize the errors of the model and of the measurement process, respectively. Both are diagonal matrices representing independent disturbances and errors.

The following steps give some suggestion to determine $T^{*}, T, \mathbf{Q}$ and $\mathbf{R}$ values

1. Simulate the LQN model with a long simulation time $T$ ' and get the maximum $95 \%$ confidence interval value among all the user response times $c i$ ' and all the performance measurements. 
2. Calculate $T^{*}$ using $T^{*}=T^{\prime} *\left(c i^{\prime} / 0.05\right)^{2}$ assuming $T^{*}$ requires $95 \%$ confidence interval is no more than $5 \%$ of the mean value.

3. Choose a value between $0.1 T^{*}$ and $T^{*}$ for $T$

4. Use fixed values for the diagonals of $\mathbf{Q}$ and adaptive values for the diagonals of $\mathbf{R}$. The values in $\mathbf{Q}$ are determined from Eq. (5.5a) or Eq. (5.5b) and the values in $\mathbf{R}$ are determined by Eq. (5.15).

The experiments of case 3 showed that it is the ratio of $\mathbf{Q}$ to $\mathbf{R}$ that matters, not the values separately, and that $\mathbf{Q}$ can be overestimated (or $\mathbf{R}$ can be underestimated) by up to 2 orders of magnitude with only a slight effect on tracking quality. If it is difficult to determine one of them, it is better to overestimate $\mathbf{Q}$ and underestimate $\mathbf{R}$.

The choice of the measurement step time $T$ may depend on the change rate. It is suggested to have a change rate not faster than a change per $10 \mathrm{~T}$.

The experiments showed that more related measurements help the tracking quality. It suggests to use measurements as much as possible for tracking, especially the ones related to the tracking parameters. For real systems, it is suggested to use end to end response times, system throughputs and processor CPU utilizations as measurements as much as possible. If it is possible to have the CPU utilization for each function, it is recommended to add them to the measurement set as well (e.g. the results in Figure 5.34 are better than the ones in Figure 5.32). 


\section{CHAPTER 6 DECISION MAKER}

The Decision Maker block in the framework uses policies, statistic methods, search algorithms or optimization approaches to configure the system to meet the QoS requirements with minimum resource costs based on the performance predictions from the Performance Model block. Different decision making strategies are designed for different system problems.

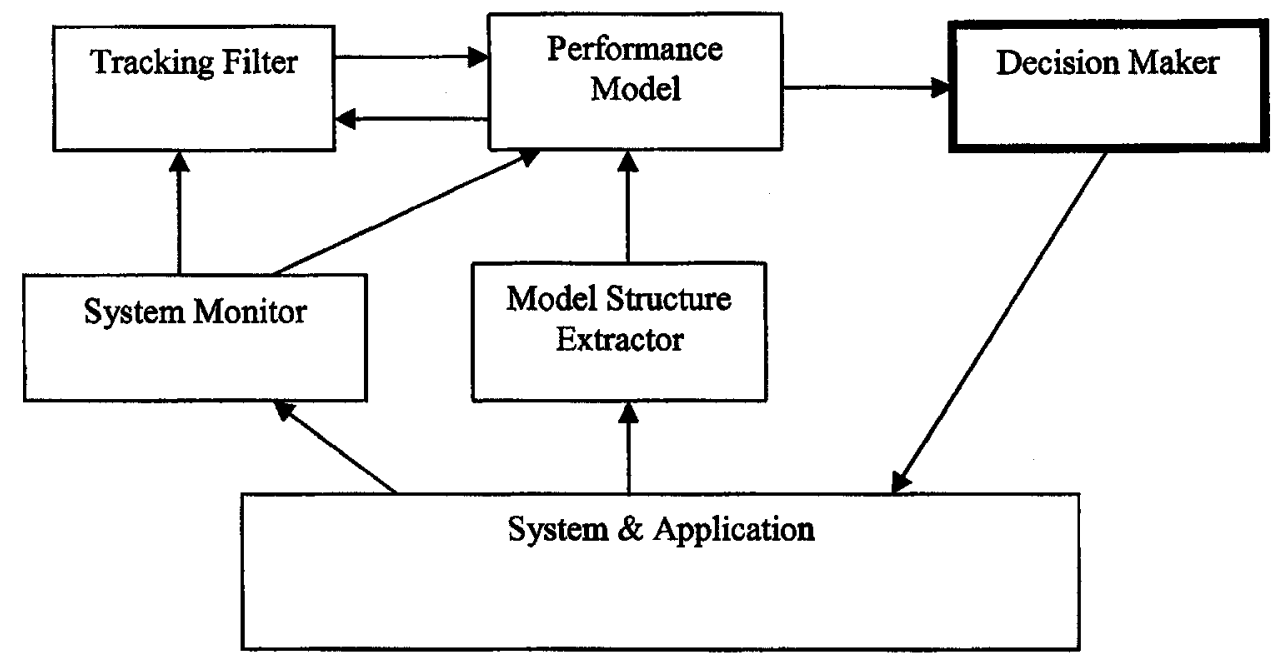

Figure 6.1 The Architecture of the Framework

The strategies used in the Decision Maker are based on the target systems with different QoS requirements and available controls. As mentioned before, the primary target systems of this research are multi tier information systems. The QoS requirements focus on the service level agreements (SLA) of the user response times. The resource costs are mainly the cost of server replicas or threads etc. The goal of the Decision Maker block is to use minimum resource costs to make the average or percentile of the user response times less than the required SLA thresholds. A cost function is defined to describe the total cost of the system for each configuration. The total cost 


$$
C_{\text {total }}=C_{S L A}+C_{\text {resource }}=\Sigma C_{S L A, i}+C_{\text {resource }}
$$

where $C_{S L A, i}$ is the cost of violating the SLA in class $i$ and $C_{\text {resource }}$ is the cost of the used resources.

If $C_{S L A, i}$ is considered as a penalty of violating the SLA, $C_{S L A, i}=0$ when the SLA is not violated. And $C_{S L A, i}$ is a positive number when the SLA is violated.

The resource cost

$$
C_{\text {resource }}=w \Sigma\left(C_{j} * N_{j}\right)
$$

where $C_{j}$ is the cost assigned to each resource type and $N_{j}$ is the number of units of this type of resource and $w$ is a weight assigned to the resource cost.

The Decision Maker block aims to find a system configuration with minimum $C_{\text {total. }}$

\subsection{Hill Climbing Algorithm}

Hill climbing [41] is a simple search algorithm to find a local optimal. It is started with a specific system state, compares the function costs of the neighbors, and moves to the neighbor state with the best function cost value. The algorithm repeats the process until the function cost value can not be improved anymore. Hill climbing algorithm can not guarantee the global optimal unless the surface of the function cost is convex. The algorithm may encounter problems if the surface of the function cost has ridges or plateau. However, the hill climbing algorithm works well to solve the server provisioning problem, admission control and threads reservation problems in the Decision Maker block.

In this thesis, it is assumed that the violation of SLA $C_{S L A}$ is much more important than the resource cost $C_{\text {resourc }}$. The resource management always tries to meet SLAs first. If all the SLAs can be met, then the system configuration with less resource cost will be chosen as the best system configuration.

In (layered) queueing network model, more server replicas and a smaller number of users usually are assumed to provide smaller response time. This is more likely to make the local optimal from the hill climbing algorithm the global optimal.

Figure 6.2 shows an example of using the hill climbing algorithm for a server provisioning problem. The state of this problem is $\left[N_{w}, N_{d}\right]$, represented by a circle. $N_{w}$ 
and $N_{d}$ are the number of replicas of the web server and database server. The function cost of each state is shown in each circle. The first number is the $C_{S L A}$ and the number in parentheses is $C_{\text {resource. }}$ The optimal state is the state with minimum $C_{S L A}$ and $C_{\text {resource }}$ (i.e. state E)

Each state $\left[N_{w}, N_{d}\right]$ has maximum four neighbor states $\left[N_{w}-1, N_{d}\right]\left[N_{w}+1, N_{d}\right]$ $\left[N_{w}, N_{d}-1\right]\left[N_{w}, N_{d}+1\right]$ if available.

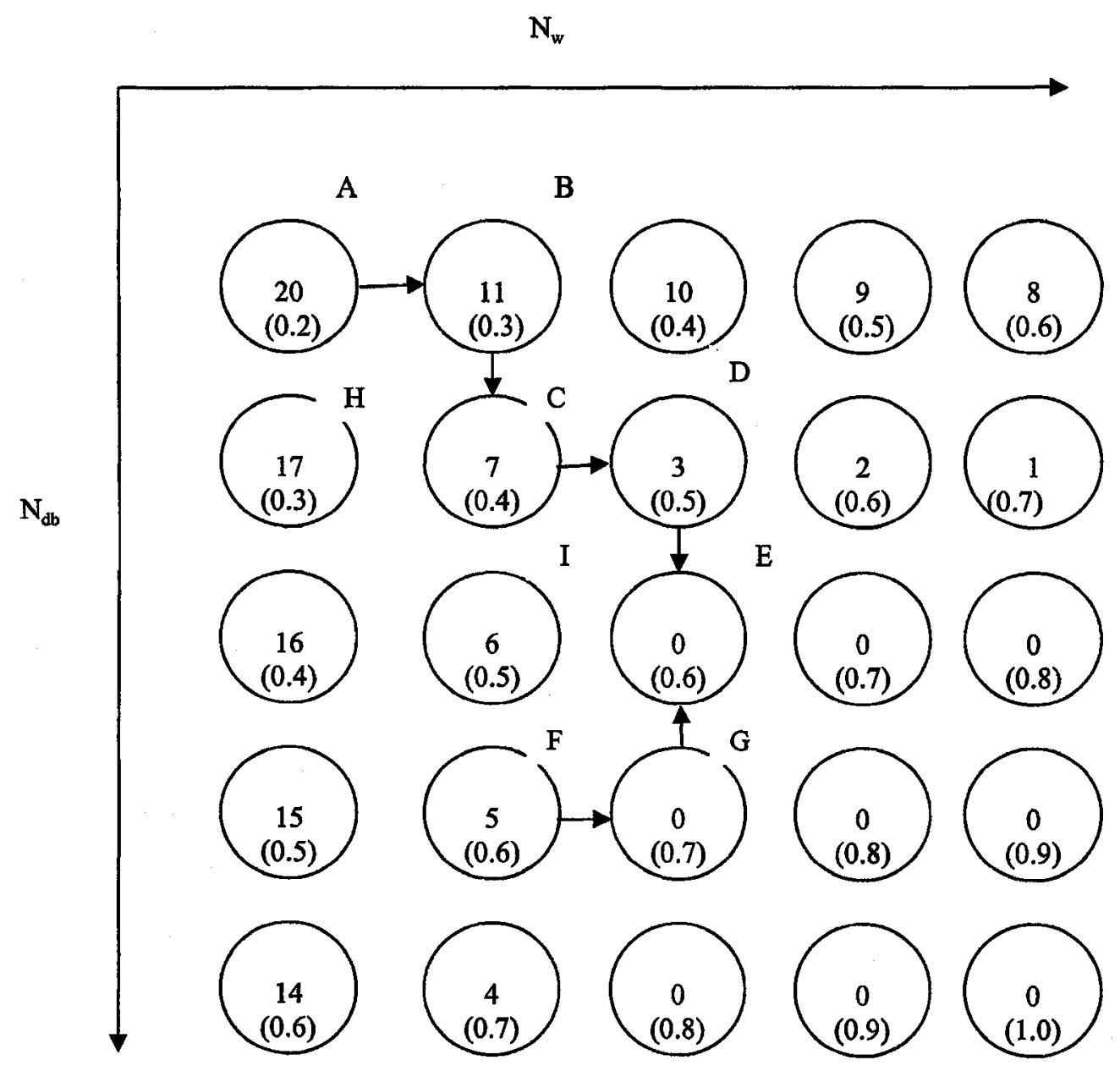

Figure 6.2 Hill Climbing for Server Provisioning

In Figure 6.2, if the initial state is state A, the hill climbing algorithm follows the path A $\rightarrow$ B $->$ C $->$ D $->$ E to reach the optimal state E. If initial state is F, it follows the path $\mathrm{F} \rightarrow \mathrm{G} \rightarrow \mathrm{E}$. 
Generally for each state, it needs to calculate the function costs of all the neighbor states and move the state with minimum function cost. However, as we know the monotonicity of the function cost in server provisioning problem, it only needs calculate function cost of half of the neighbor states. For example, the state $C$ in Figure 6.2 has 4 neighbor state B, $\mathrm{H}, \mathrm{D}$ and I. Because $C_{S L A}>0$, and the object is to reach a state with $C_{S L A}$ $=0$, only state $\mathrm{D}$ and $\mathrm{I}$ is able to make $C_{S L A}$ smaller. The states $\mathrm{B}$ and $\mathrm{H}$, with less number of servers, can only make $C_{S L A}$ Bigger. Thus, it is able to make the right decision without considering the state $\mathrm{B}$ and $\mathrm{H}$.

\subsection{Provisioning and Admission Control in a Simulated Web Server System (WSS)}

This section includes the experiment results of a simulated web server system to show the effectiveness of LQN performance model and the hill climbing algorithm for dynamic resource management using provisioning and admission control.

\subsubsection{Simulated Web Server System}

The LQN model of the web server system is shown in Figure 2.2. A simulator simulates the behavior of the LQN model of the time-varied web application described in section 2.3. The simulated system has two clusters: a web server cluster and a database cluster. The two clusters share the same server pool.

In real web based service systems, different classes of user or transactions may consume different combinations of web server resources and database resources. The simulated system was designed to have 80 different combinations of web server service demand and database server service demand. Each combination lasted 10 measurement steps. There were 800 measurement steps in total. Figure 6.3 shows the $S_{w}$ and $S_{d}$ varying with the time. The thick line is $S_{d}$ and the thin line is $S_{w}$. There are a number of times the service demands jump from a low level to a very high level abruptly. For example, $S_{d}$ jumps from $10 \mathrm{~ms}$ to $70 \mathrm{~ms}$ at step 271 and $S_{w}$ jumps form $5 \mathrm{~ms}$ to $35 \mathrm{~ms}$ at step 561 . Those service demands combination can check how fast the controller can respond to big changes.

There are two common strategies to meet the SLAs when the workload is too high for 
the current system configuration: provisioning control and admission control. The provisioning control strategy tries to add more server replicas to meet SLA. If no more server replicas are available, admission control can be used to allow only part of the users to access the system to make sure the served users meet QoS requirements. A controller was designed to either use provisioning control or admission control to achieve QoS requirements under different workload conditions. The SLA was set as the mean user response time $R \leq 400 \mathrm{~ms}$.

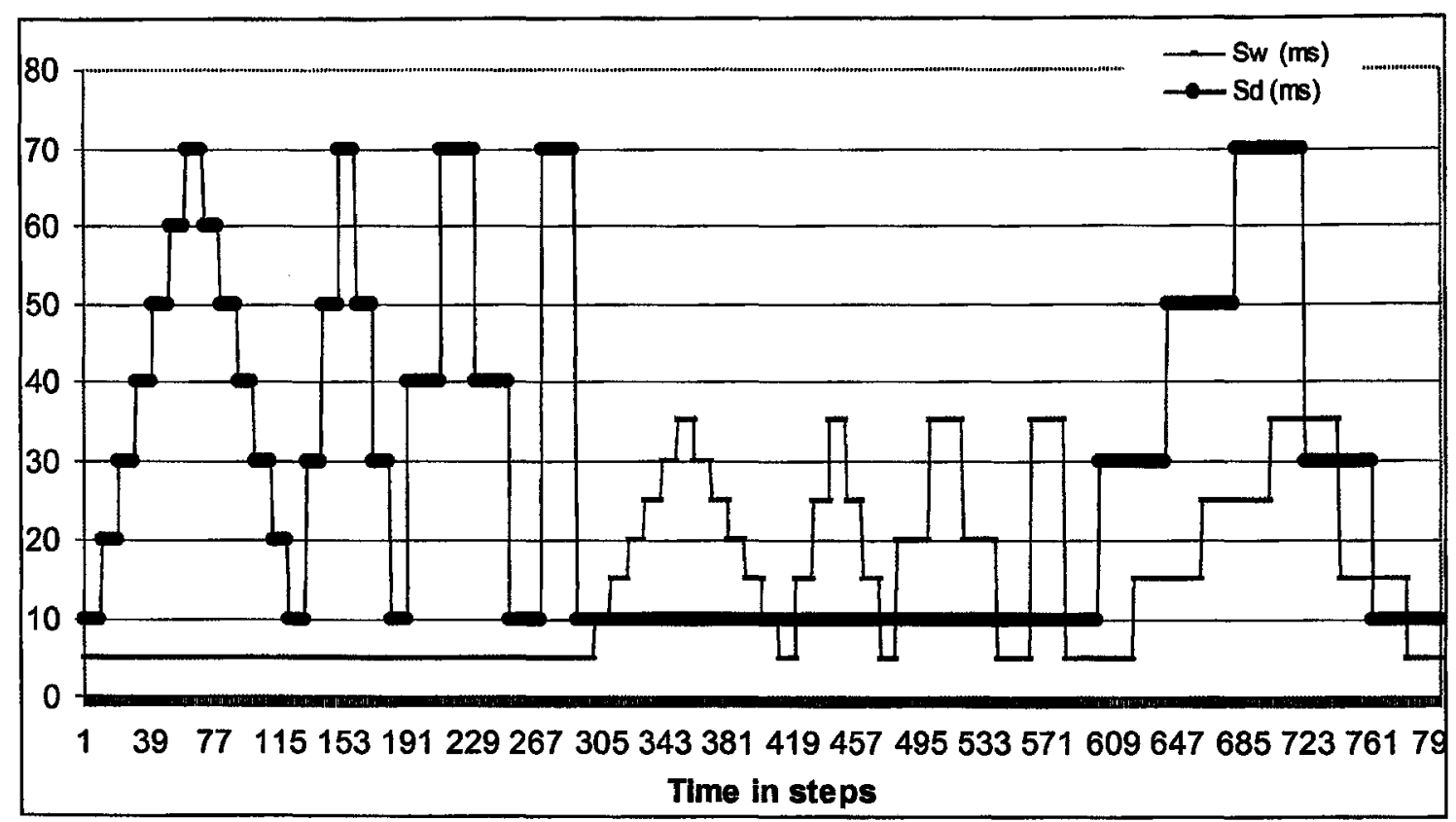

Figure 6.3 Values of $S_{w}$ and $S_{d}$ Varied with Time (WSS)

\subsubsection{Provisioning Control of the Web Server System}

This part uses different provisioning controls to evaluate the dynamic control

- The control is evaluated by $C_{S L A}$, the cost of violating the SLA, defined as a sum over all 800 measurement steps of the SLA violation, normalized by the threshold amount:

$$
C_{S L A}=\sum_{\mathrm{i}=1,800} \max \left(R_{i}-400,0\right) / 400
$$

- The resource cost is evaluated by average value of sum of the number of web 
server replicas $N_{w}$ and database replicas $N_{d}$ over 800 measurement steps. The weight parameter $w$ is set to 0.1 to make $C_{S L A} \gg C_{\text {resource }}$

$C_{\text {resource }}=0.1^{*} \sum_{\mathrm{i}=1,800}\left(N_{w, i}+N_{d, i}\right) / 800$

The simulation experiments drive the system through a wide-ranging series of demand states in a deterministic and repeatable fashion, which makes it easier to compare the quality of adaptation under different strategies. Dynamic provisioning is evaluated for different factors that may constrain the system in practice:

- Static provisioning with a wide range of fixed provisioning values $N_{w}$ and $N_{d}$.

- Perfect provisioning with perfect predicted demand estimates to calibrate the model;

- Dynamic provisioning using hill climbing algorithm with estimates with some errors.

Static provisioning (WSS)

\begin{tabular}{|c|c|c|c|c|c|}
\hline \multirow{2}{*}{$\begin{array}{c}\text { DataServer } \\
\text { replicas (Nd) }\end{array}$} & \multicolumn{5}{|c|}{ WebServer replicas (Nw) } \\
\cline { 2 - 6 } & 1 & 2 & 3 & 4 & 5 \\
\hline 1 & 1326.7 & 639.9 & 552.9 & 550.8 & 548.3 \\
\hline 2 & 956.2 & 122.1 & 17.9 & 17.4 & 16.4 \\
\hline 3 & 945.7 & 107.1 & 0.0 & 0.0 & 0.0 \\
\hline 4 & 945.1 & 106.6 & 0.0 & 0.0 & 0.0 \\
\hline 5 & 941.5 & 105.5 & 0.0 & 0.0 & 0.0 \\
\hline
\end{tabular}

Table 6.1 $C_{S L A}$ for different WebServer and DataServer replicas

With static provisioning, the numbers $\left(N_{w}, N_{d}\right)$ of replicas of the WebServer and DataServer are constant over the 800 measurement steps. Table 6.1 shows the $C_{S L A}$ value for each combination of WebServer and Database replicas. It shows that the system must have at least 6 servers to meet SLA all the time in the experiment. A larger number of servers is not always has smaller function cost. The number of replicas must be assigned properly to have smaller $C_{S L A}$. For example, the configuration $\left(N_{w}=2, N_{d}=5\right)$ has 7 servers in total. However, its $C_{S L A}$ is 105.5 which is much worse than the configuration $\left(N_{w}=3, N_{d}=3\right)$ which has 6 servers in total and $C_{S L A}=0.0$. 


\section{Perfect provisioning with perfect demand predictions (WSS)}

Static provisioning causes high resource costs. It must keep the numbers of the web server and database server replicas to meet the SLA at worse case all the time. If the actual demand values $S_{w}$ and $S_{d}$ are known exactly at the time they change, and the optimal provisioning can be determined and instantaneously installed, we have the best possible control and the SLA is met all the time $\left(C_{S L A}=0\right)$ with the minimum necessary processors.

In the experiment, the average number of servers necessary was 3.08 just a little more than half of the requirement for static provisioning.

\section{Dynamic provisioning using hill climbing algorithm (WSS)}

This strategy uses the performance model and the hill climbing algorithm to do the provisioning. The flow chart of the algorithm is shown in Figure 6.4. The hill climbing is used to figure out the neighbor state with the smallest function cost. Two thresholds SLA_High and SLA_Low are used to determine whether the provisioning algorithm should stop or not.

In the Web Server System (WSS), SLA_High was set to $400 \mathrm{~ms}$, which is the SLA of WSS. SLA_Low was set to $300 \mathrm{~ms}$. This threshold is used to avoid oscillation in dynamic control.

Figure 6.5 shows the measured response time and total servers for 800 measurement steps.

The dynamic provisioning in this part is a reactive approach. It did not predict the service demand of the next measurement step. As shown in Figure 6.3, the real service demands may jump from a very low level to a very high level. It is impossible to meet SLA all the time. However it is able to lower the measurement response time as fast as possible even with server demands changing sharply. There are 18 times the SLA were violated. Among those 18 times, the controller added 2 servers at a time twice. At measurement step 271, the measured response time was lowered from $1615 \mathrm{~ms}$ to $184 \mathrm{~ms}$ 


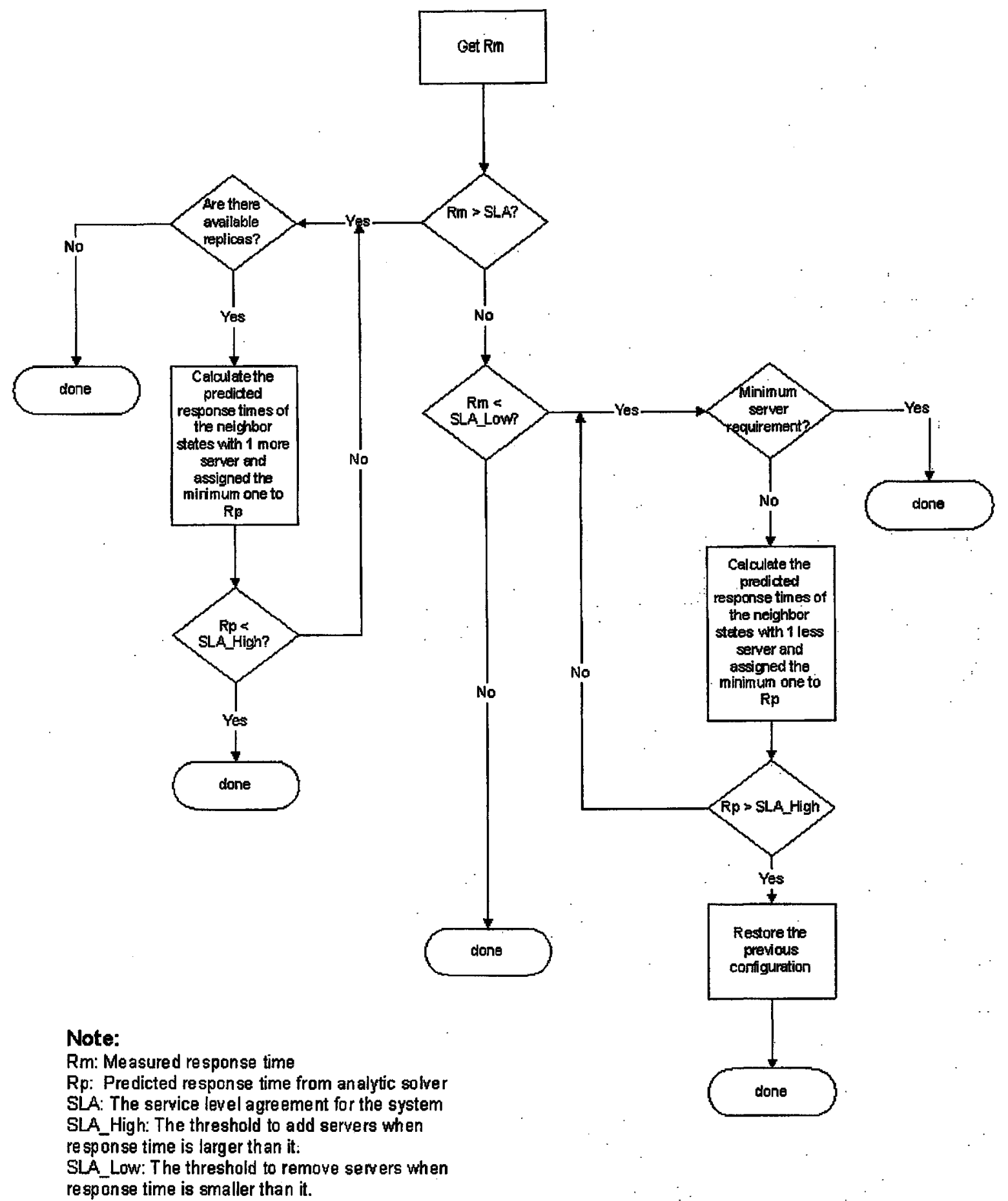

Figure 6.4 Flow Chart of Provisioning Control

after adding 2 database replicas. And at measurement step 561, the measured response time was lowered from $2323 \mathrm{~ms}$ to $355 \mathrm{~ms}$ after adding 2 web server replicas. Among the other 16 times, the controller added 1 server at a time to adapt to the heavy workload. 
There are another 16 times that the measured response time was lower than the threshold SLA_Low. Among the 16 times, there were 4 times that the controller removed 2 servers at a time. Among the other 12 times, only 1 server was removed at a time. The provisioning algorithm works well to figure out which cluster should be chosen for provisioning and how many replicas should be involved.

In the simulated web server system, the dynamic provisioning has a $C_{S L A}$ value of 18.50 over the 800 steps, with an average of 3.09 servers. Thus the resource cost (the average number of servers in user over 800 steps) is very close to the optimal value of 3.08 , with a small $C_{S L A}$. The static provisioning strategy needs at least 5 servers to have a similar $C_{S L A}$ value.

With some additional knowledge to predict the CPU demand change, the $C_{S L A}$ might be smaller. In real systems, it is possible to get statistical data to guide the workload prediction although perfect prediction is unavailable.

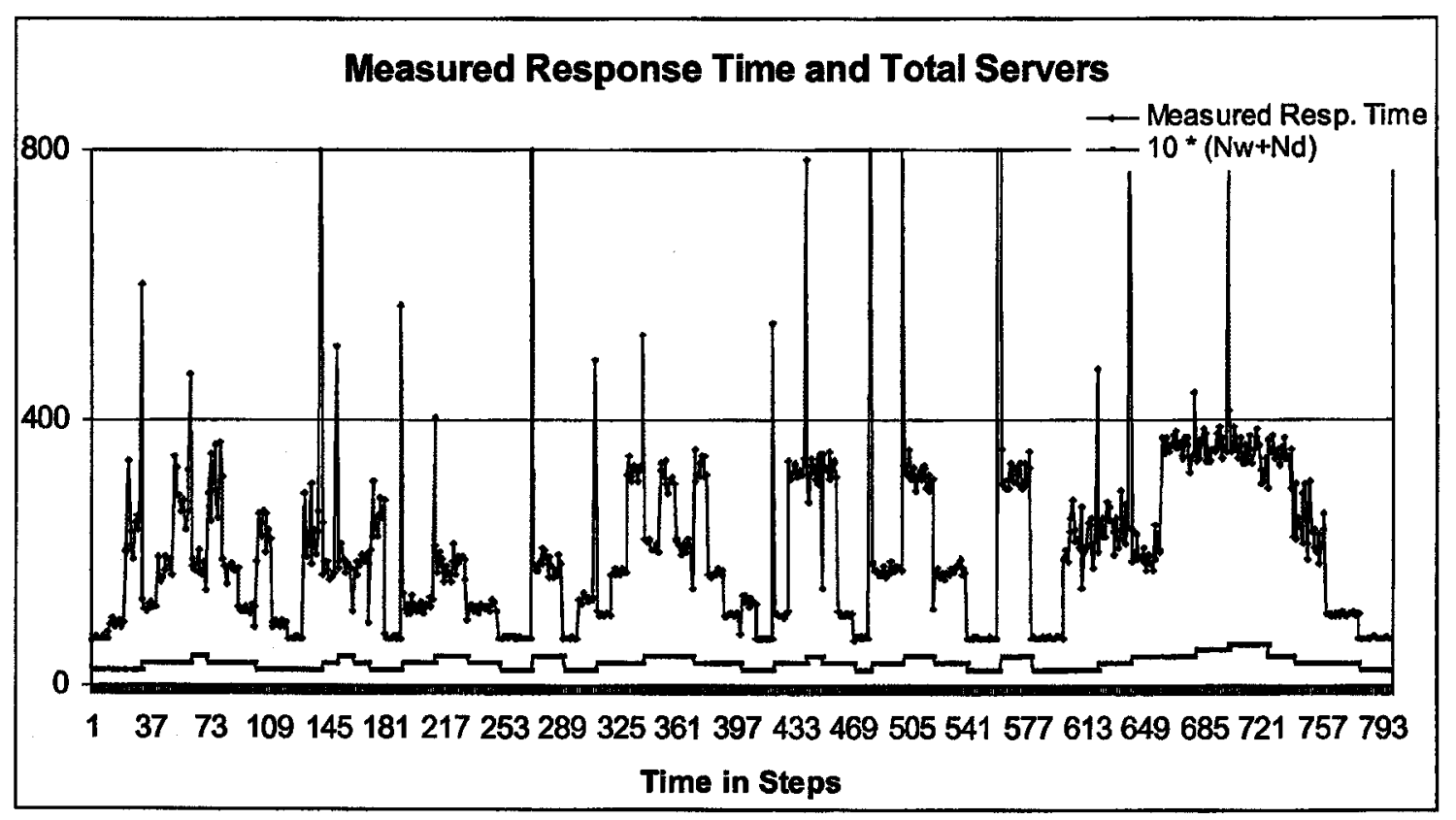

Figure 6.5 Measured Response Time and Total Servers for Dynamic Provisioning

\subsubsection{Admission Control of the Web Server System}

The hill climbing algorithm can be used in admission control as well. In this case, the 
state of the system is the number of users in the system [ $N]$. For each system state, it has two neighbor states: $[N-5]$ and $[N+5]$ (assuming the number of users must change at a value of 5 at least. If there are no more server replicas available, when the user response time exceeds the SLA, only part of the users are accepted to be served. The control algorithm is similar to the in dynamic provisioning. The only difference is that the hill climbing algorithm is used to get the optimal number of users $(N)$ instead of the optimal configuration of $\left[N_{w}, N_{d}\right]$ in the provisioning control.

Figure 6.6 shows the measured response time and number of users when admission control were applied. The measured response time was around the 400 , which is SLA_High.

Experiment with SLA_High $=400 \mathrm{~ms}$ and SLA_Low $=300 \mathrm{~ms}$

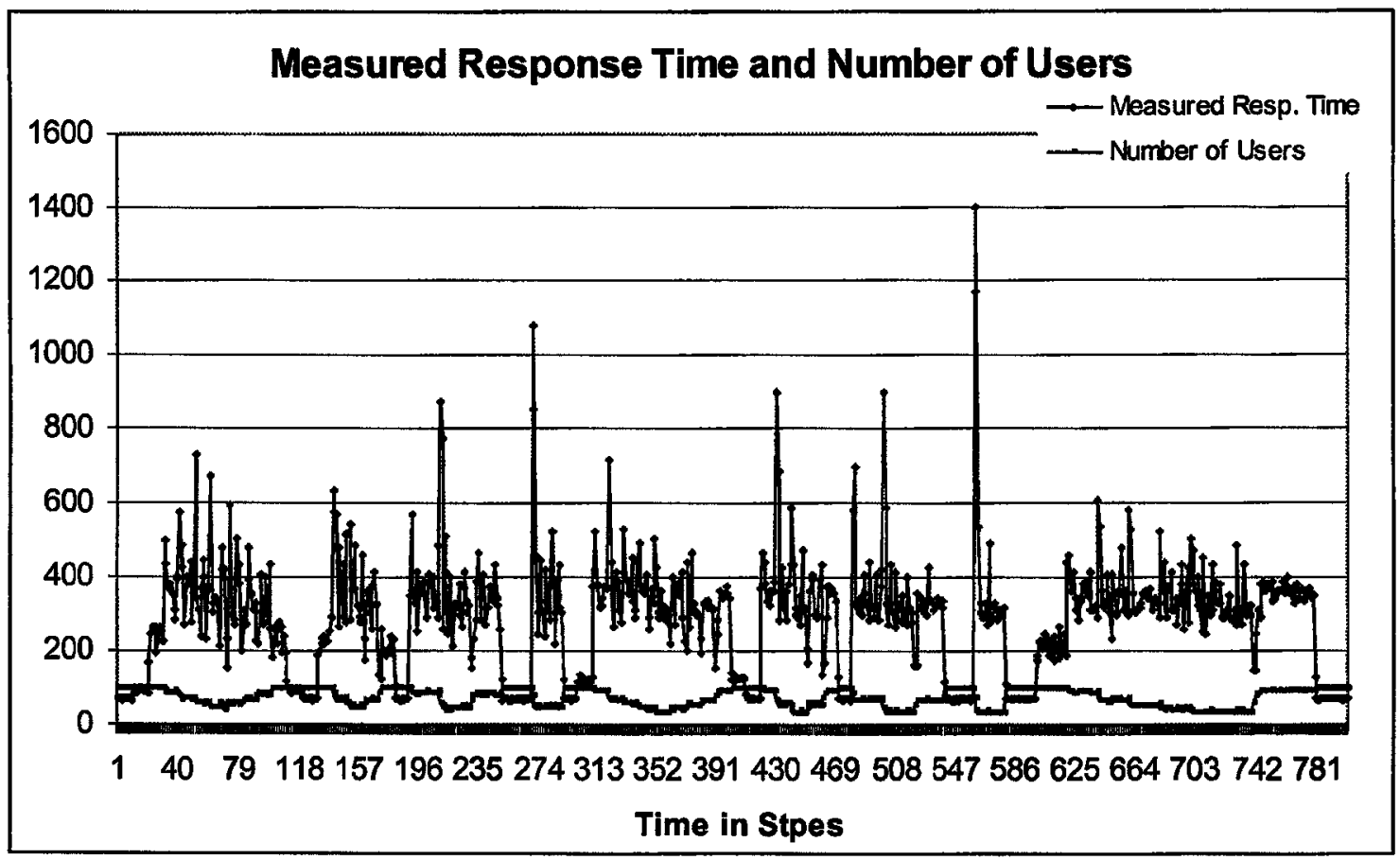

Figure 6.6 Measured Response Time and Number of Users for Admission Control of WSS (SLA_High $=400 \mathrm{~ms}$ )

Figure 6.6 shows the measured response time and number of users accepted using the server demands shown in Figure 6.3. Among the 800 measurement steps, there were 118 
times that the measured response time was more than $400 \mathrm{~ms}$. The function cost $C_{S L A}$ was 31.847 and the average number of users accepted was 72.8 . Because the tracking filter and analytic solver may have prediction errors, the measured response time may be more than SLA_High even though the predicted response time from the analytic solver is less than SLA_High. A good way to solve this problem is to use a guard band for protection.

Experiment with SLA_High $=350 \mathrm{~ms}$ and $S L A \_L o w=300 \mathrm{~ms}$

In this experiment, $S L A \_H i g h$ was not $400 \mathrm{~ms}$ anymore. The value of $S L A_{-} H i g h$ was set to $350 \mathrm{~ms}$ (assuming the tracking filter and analytic solver may have a prediction error around 15\%). Figure 6.7 shows the response time and the number of users over 800 measurement steps. Among the 800 measurement steps, there were 59 times that the measured response time was more than $400 \mathrm{~ms}$. The function cost $C_{S L A}$ was 22.176 and the average number of users accepted was 71.5

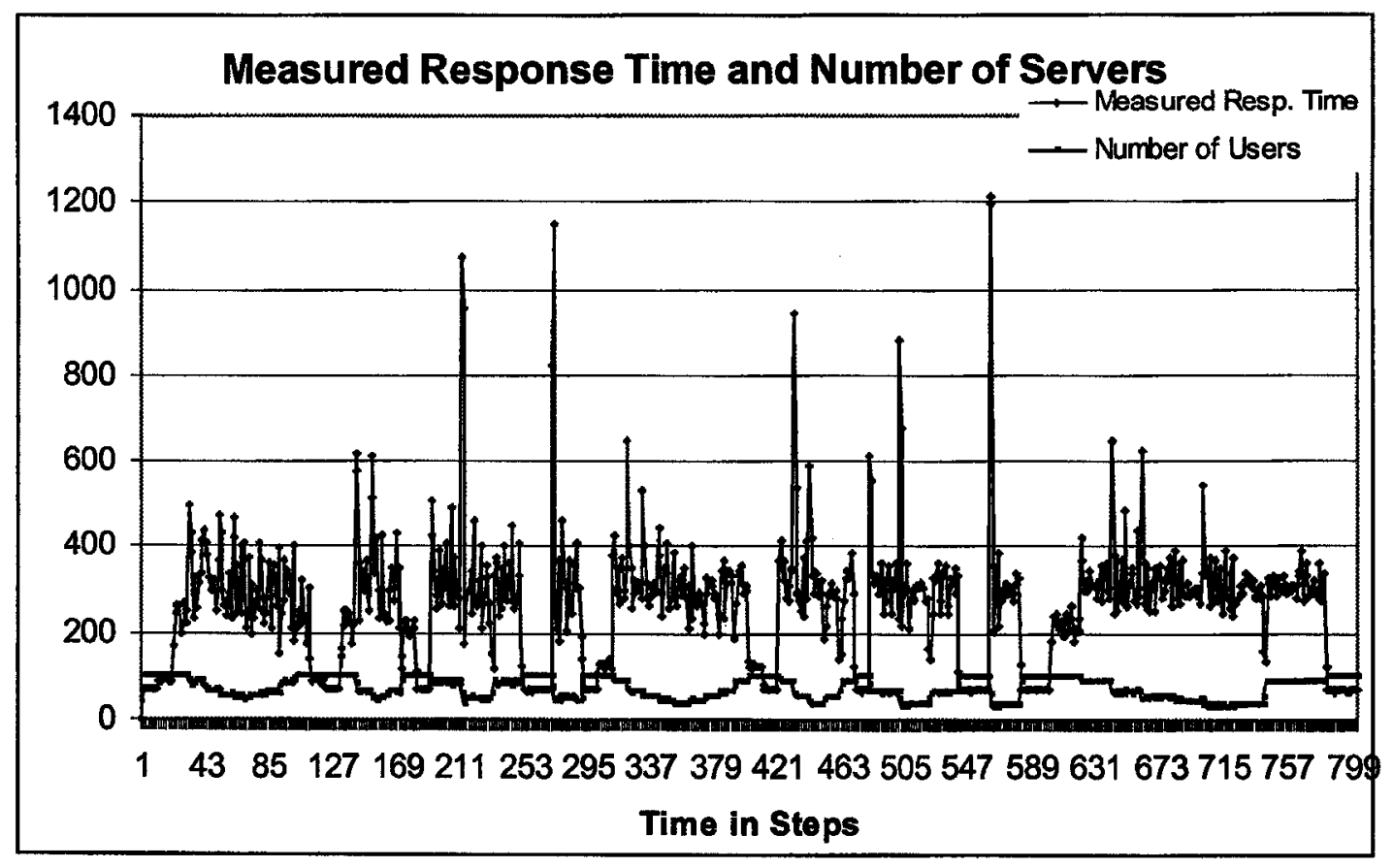

Figure 6.7 Measured Response Time and Number of Users for Admission Control of WSS (SLA_High $=350 \mathrm{~ms}$ )

By using a guard band, the number of violating the SLA decrease 50\% and CSLA was improved significantly as well. The number of users accepted was slightly lower. 


\subsection{Provisioning and Threads Adjustment for a Simulated Multi Tier System with Two Classes of Users}

A simulated multi tier system was built based on the LQN model shown in Figure 5.31. The behavior of the simulated system was described in section 6.5. This simulated system has two classes of users: User and Manager. Both classes send requests to App task with different probabilities. The client workload has a combination of $N_{\text {twer }}$ and $N_{\text {manager }}$ shown in Figure 6.8. There are 28 different combinations and each combination lasts 10 measurement steps. There are 280 measurement steps in total. The multi tier system has three clusters: AppProc, DB1Proc and DB2Proc. All the three clusters share the same server pool. The state of the system is a vector of $\left[N_{A p p}, N_{D B 1}, N_{D B 2}\right]$. The SLA for the two classes of users are: SLA $_{1}($ User $)=1 \mathrm{~s}, \mathrm{SLA}_{2}($ Manager $)=5 \mathrm{~s}$.

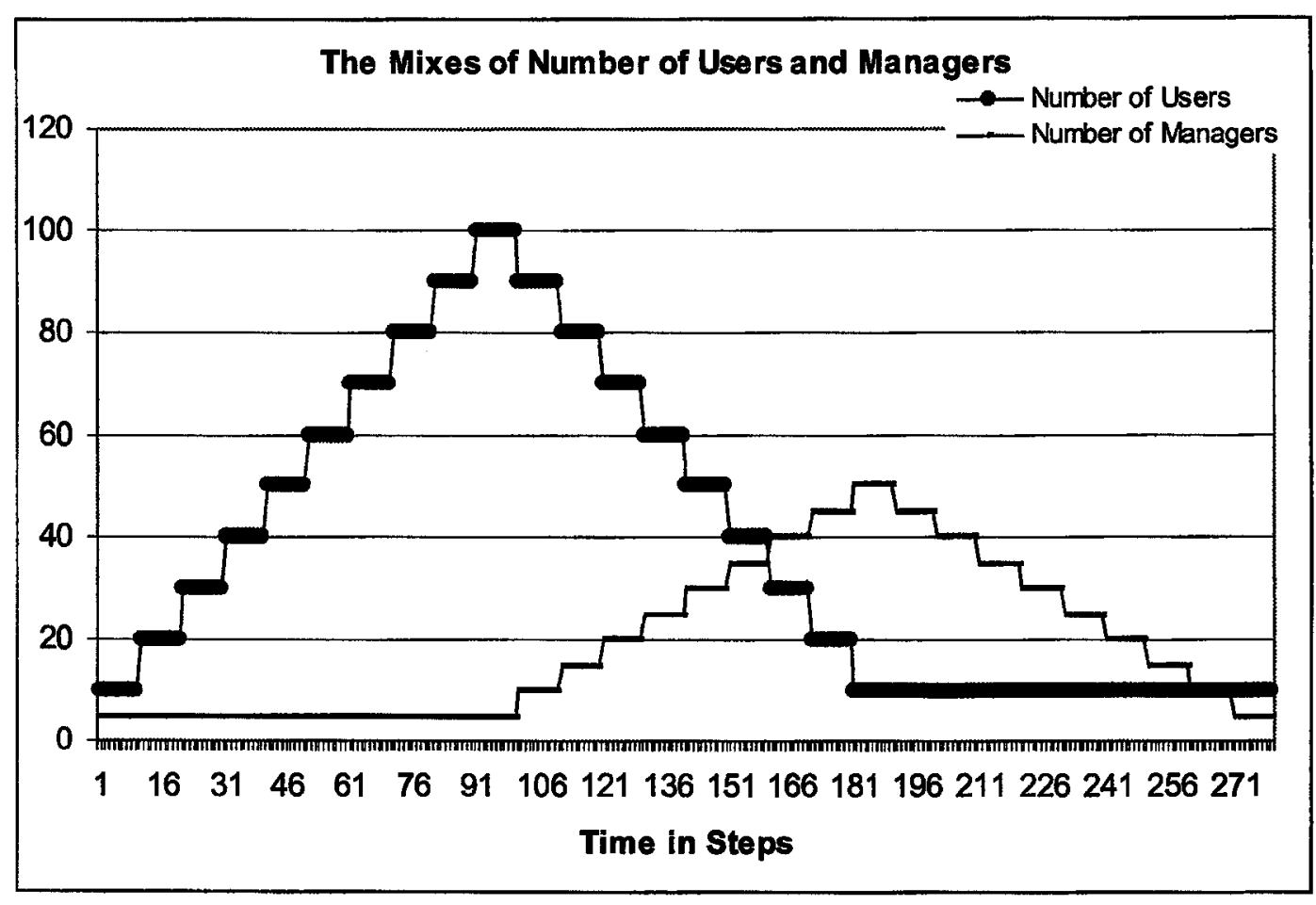

Figure 6.8 Values of Class Mix Varied with Time (Multi tier System with 2 Classes of Users)

Based on Eq. (6.1), the cost of violating SLA : $C_{S L A}=C_{S L A I}+C_{S L A 2}$

$$
\begin{aligned}
& C_{S L A 1}=\sum_{\mathrm{i}=1,280} \max \left(R_{\text {user }, i}-1\right) / 1 \\
& C_{S L A 2}=\sum_{\mathrm{i}=1,280} \max \left(R_{\text {manager }, i}-5\right) / 5
\end{aligned}
$$


The thresholds of SLA1 and SLA2 are: SLA1_High $=1 \mathrm{~s}$, SLA2_High $=5 \mathrm{~s}$, SLA1_Low $=0.8 \mathrm{~s}$ and SLA2_Low $=4 \mathrm{~s}$.

\section{Experiment with SLA1_High = 1s and SLA2_High = 5s (Provisioning Control only)}

By applying the hill climbing algorithm, the measured response times of two classes and total servers over 280 measurement steps are shown in Figure 6.9. The upper curve is the total number of servers and the lower two curves are the measured User response time (the thick one) and measured Manager response time (the thin curve). The cost of violating the SLA $\left(C_{S L A}\right)$ is 1.70 and the average number of servers is 3.9 .

Due to the tracking filter and analytic solver prediction errors described in section 6.2.3, the measured response time and predicted response time may have some difference. If they are around the threshold, it may lead to dithering as shown in Figure 6.9.

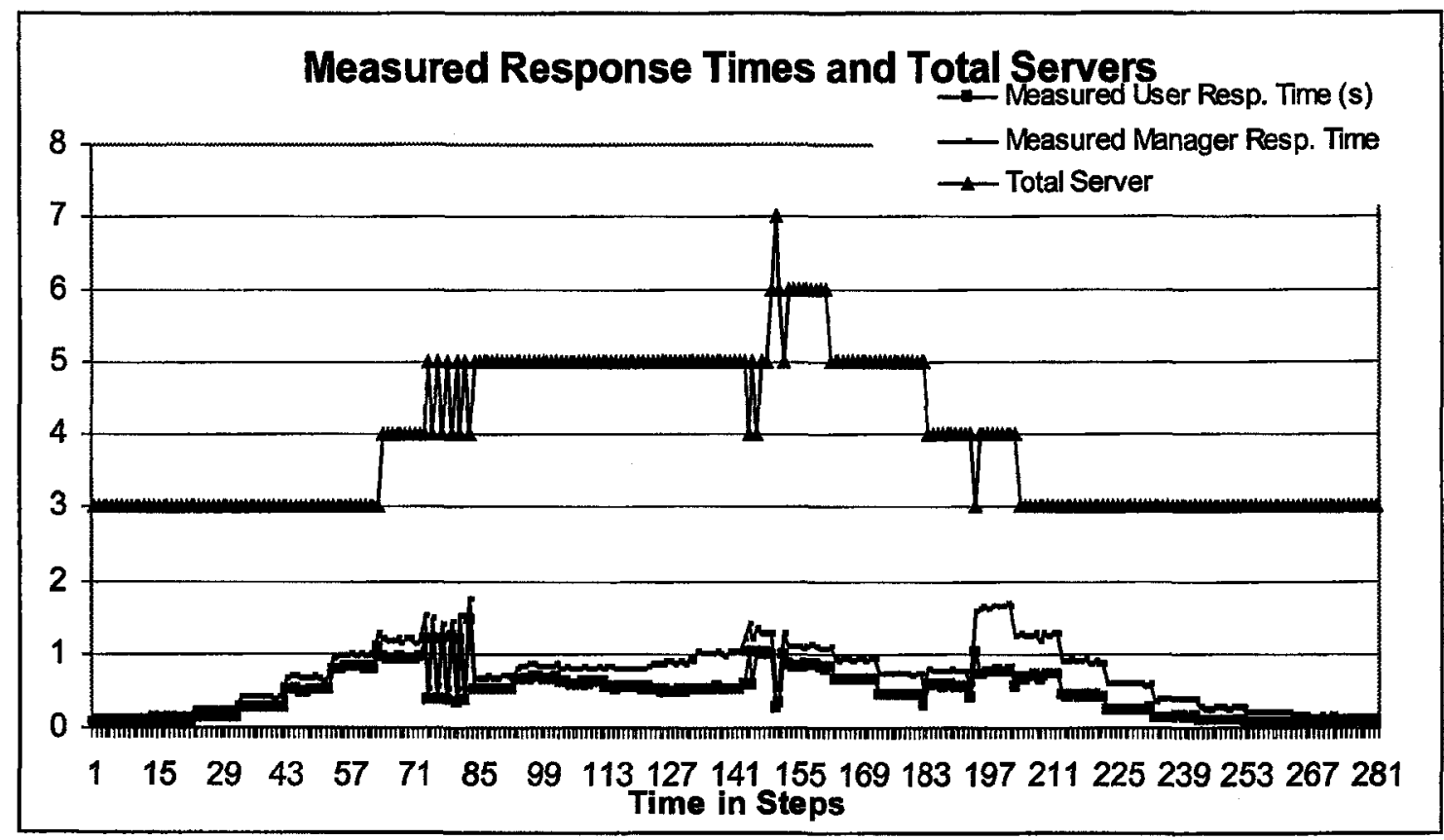

Figure 6.9 Measured Response Times and Number of Users of Multi tier System with Provisioning Control Only (SLA1_High = 1s and SLA2_High $=5 \mathrm{~s}$ )

\section{Experiment with SLA1_High $=0.9 \mathrm{~s}$ and SLA2_High $=4.5 \mathrm{~s}$ (Provisioning Control only)}

Another experiment result using guard band shows (Figure 6.10) much more smoother provisioning. The $C_{S L A}$ was 0.292 and average number of server was 4.0 . The use of guard 
band made $C_{S L A}$ much smaller. It helped the provisioning avoid dithering.

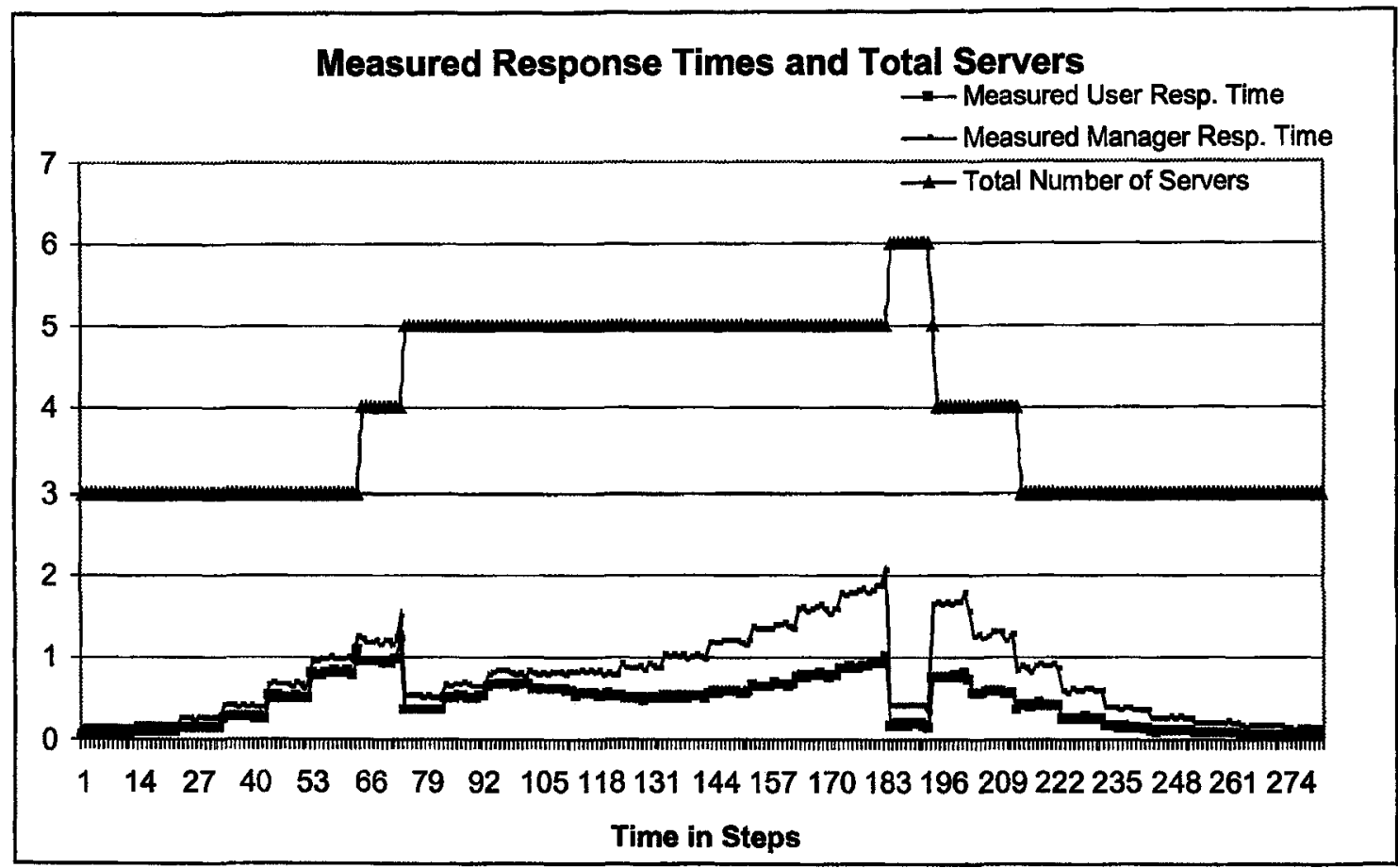

Figure 6.10 Measured Response Times and Number of Users of Multi tier System with Provisioning Control Only (SLA1_High = 0.9s and SLA2_High $=4.5 \mathrm{~s}$ )

\section{Experiment with both Provisioning Control and Threads Adjustment}

In this multi tier system, the class User and Manager compete for the resources (e.g. the threads in $A p p$ ). In Figure 6.9, the measured response time of User and Manager are very close most of the time. However, the $\mathrm{SLA}_{1}$ and $\mathrm{SLA}_{2}$ are quite different. The measured response time of User is very close to $\mathrm{SLA}_{1}$, and the measured response time of

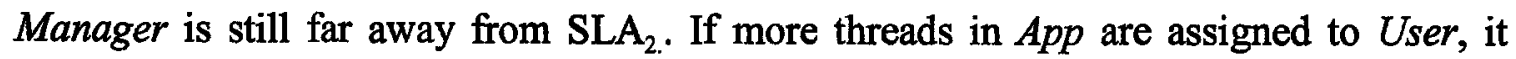
might be able to meet SLAs with a smaller number of servers.

A heuristic algorithm was designed to allocate the resources for the multi tier system with two classes of users. It first used the hill climbing algorithm to adjust the number of threads in App task for two classes of users before provisioning control. A metric $\max \left(R_{1, i} / S L A_{1}, R_{2, i} / S L A_{2}\right)$ was used to guide the thread adjustment where $R_{1, i}$ and $R_{2, i}$ are the measured response time of $U$ ser and Manager class at step $i$. It aimed to get a thread assignment with the minimum. In experiment results shown in Figure 6.11 had $C_{S L A}=$ 
0.04 and average number of servers 3.89. Both metrics were better than the one using provisioning control only. The threads adjustment improved both the cost of violating the SLA and the resource cost in this experiment. It does help the dynamic resource management.

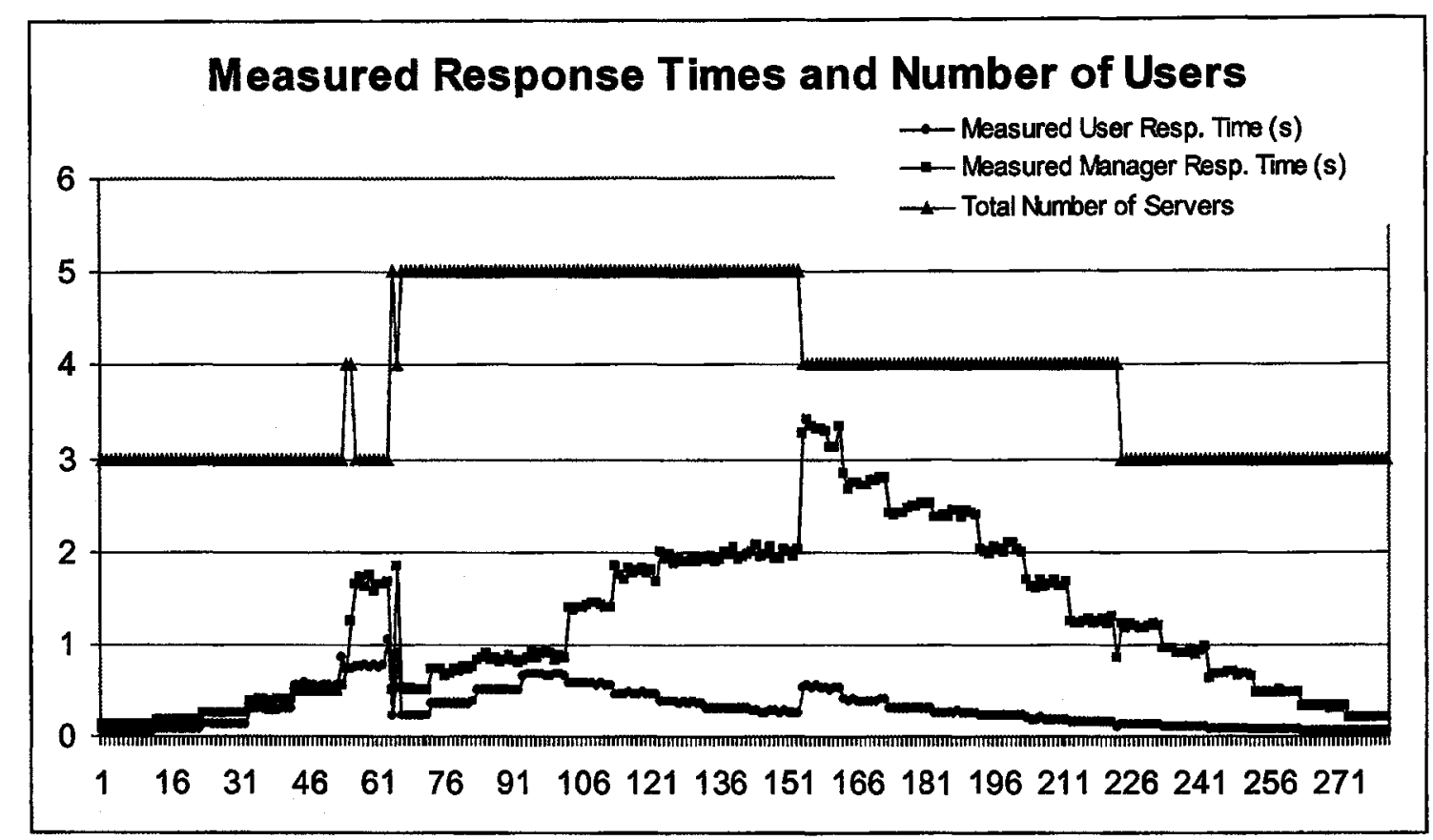

\section{Figure 6.11 Measured Response Times and Number of Users (Multi tier System with Both Provisioning Control and Threads Adjustment)}

\section{Summary}

The provisioning control and admission control are quite common mechanisms for allocating resources dynamically. If there is more than one class of users compete for the resources, the threads adjustment may help as well under some conditions. The Decision Maker and the performance model have been shown to be capable of adjusting all three of these factors (admission control, provisioning, and thread adjustment).

The approach used in the chapter is able to bring the response time from a very big value down to the SLA threshold at a step by adding enough resources.

Due to the prediction errors from the tracking filter and analytic solver, the adaptive control may have dithering problem if the SLA is simply used to guide the control. The 
use of a guard band is useful to make the adaptive control much smoother. A $15 \%$ guard band is able to decrease the number of violations by half. 


\section{CHAPTER 7 CASE STUDY}

This chapter applies the framework for automated resource management described in section 1.2 (Figure 1.2) to a real system with Trade 6 benchmark application. A performance model is validated on this real system. Server provisioning was implemented to maintain the SLA. Some real problems such as switching delay were considered when TPM was involved in provisioning.

\subsection{The Trade 6 Benchmark Application}

Figure 7.1 shows a prototype system using Trade 6 benchmark application [69]. The Trade 6 benchmark application is a WebSphere end-to-end benchmark and performance sample application developed by IBM. It is a stock trading application and allows you to buy and sell stocks, to check your portfolio, register as a new user, and so on. The application is able to generate different behaviors randomly with specific probabilities.

The clients in the system are simulated by synthetic workload generator. They access a servlet of Trade 6 application and the servlet generates different requests randomly according to the benchmark. Those requests are distributed to an application server cluster by the web server. The web server works mainly as a load balancer in this system. All the application servers in the cluster share the same database server. The system uses IBM HTTP Server 6.0 as the web server, IBM WebSphere® Application Server 6.0 as the application server and IBM DB2@ Universal Database ${ }^{\mathrm{TM}}$ (DB2 UDB) Version 8.2 as the database server. The operating systems are Windows $@ X P$ and run on IBM PCs with Intel® Pentium $\otimes 2.0 \mathrm{GHz}$ and $1 \mathrm{~GB}$ of memory. In order to manage the application server cluster dynamically with different numbers of clients, an autonomic manager was implemented to manage a server pool through IBM Tivoli Provisioning Manager (TPM) Version 5.3, is able to automate the actual server provisioning by executing specific workflow scripts. The autonomic manager is able to monitor to run-time performance metrics such as the response times, invoke rates, CPU utilizations etc from WebSphere application server using Java Management Extensions (JMX) technology. Those 


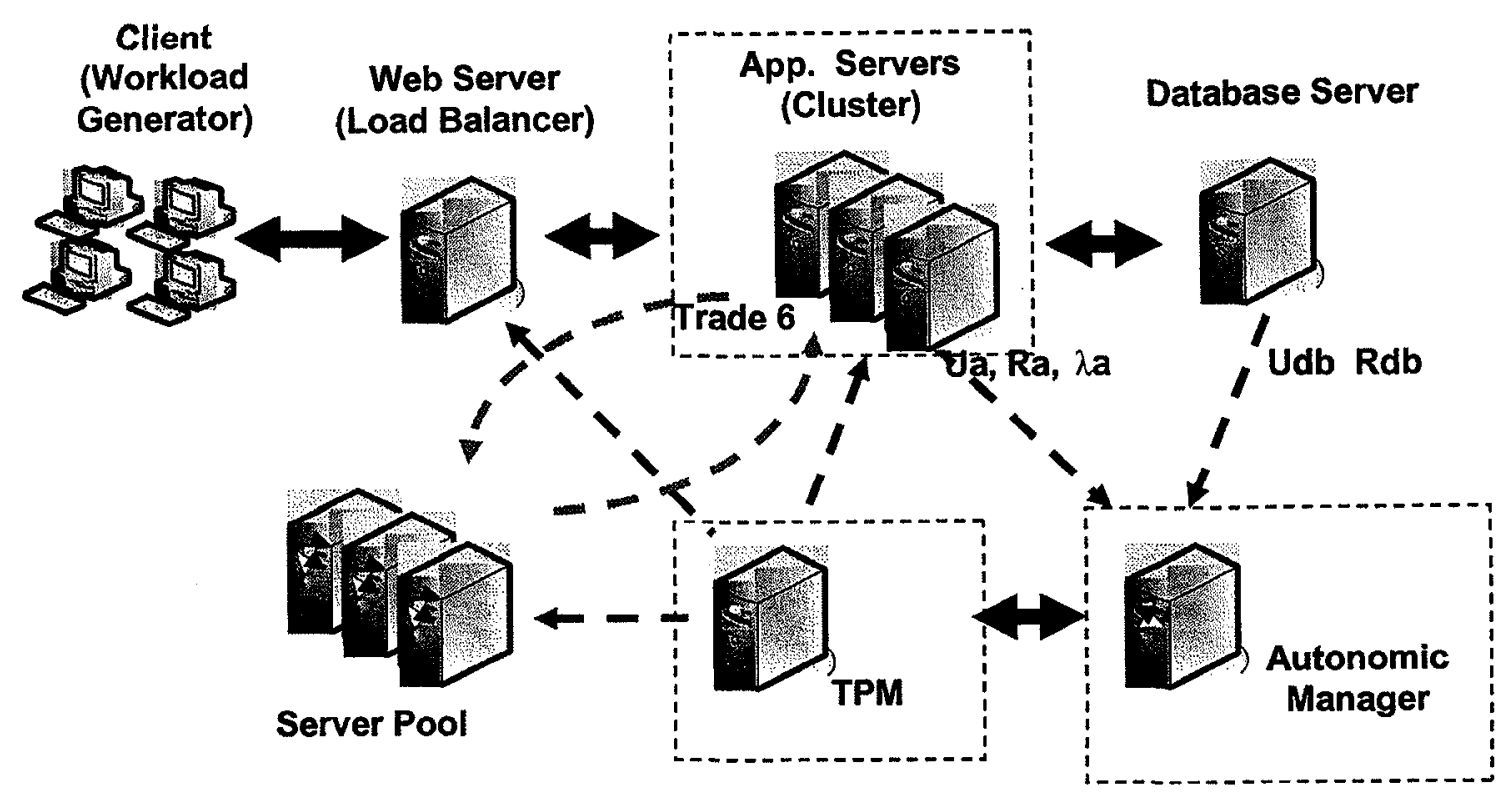

Figure 7.1 The System Architecture of the Trade 6 Setup

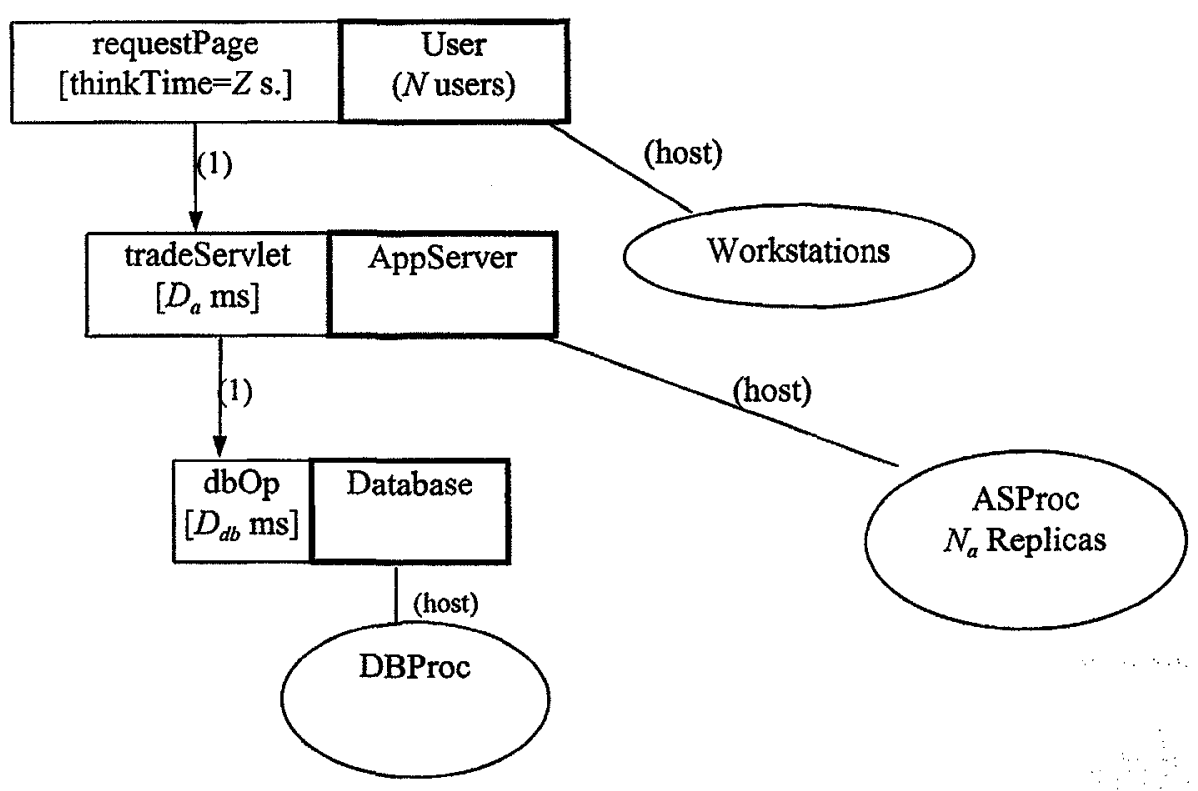

Figure 7.2 The LQN Model of the Real System

measurements are fed to the Kalman filter to estimate and update the model parameters. A new system configuration will be determined according to the performance prediction 
and SLA. TPM executes workflow scripts to reconfigure the system. Then the servers in the server pool will be added to / remove from the cluster as required.

\subsection{Evaluation of the performance model on the Trade 6}

\section{Application}

A performance model (shown in Figure 7.2) was built to represent the system architecture and behavior. In this model, the web server (load balancer) was not modeled separately as we were more interested in the application servers and we lacked the tools to get the required measurements for the web server.

The measurement vector was set as $\mathbf{z}=\left[R_{a}, U_{a}, R_{d b}, U_{d b}, \lambda_{a}\right]$ where $R_{a}$ and $R_{d b}$ are the response times of the application server and the database server. $U_{a}$ and $U_{d b}$ are the utilizations of the application server and database server. $\lambda_{a}$ is the throughput of the application server which is the same as the throughput of the system. All those measurements can be collected through JMX APIs which are implemented in WebSphere Application Server 6.0. The tracking parameter vector was set as $\mathbf{x}=\left[Z, D_{a}, D_{d b}\right]$ where $D_{a}$ and $D_{a b}$ are the service demands of the application server and database server. $Z$ is the think time of the client.

In the experiment, the actual number of clients $N$ first went from 2 to 30 , in increments of 2 . Then it went from 30 back to 2 , in decrements of 2. $N$ was assumed as a known parameter in this experiment. Think time $Z$ was a constant of $1000 \mathrm{~ms}$. Although it is not possible to know the exact values of $D_{a}$ and $D_{d b}$, the tracked user think time and the real value could be compared to evaluate how the performance model fit the system.

Figure 7.3 shows the tracking results of the experiment. The first curve (on the top) with a few spikes is $1 / 10$ of the tracked user think time $Z$. The tracked think time $Z$ was around $1020 \mathrm{~ms}$ which was a little bit above the actual value (i.e. $1000 \mathrm{~ms}$ ). This small difference might come from the possible network delay in the real system. The second and the third curves (they are almost the same and difficult to distinguish in Figure 7.3) are the measured and predicted response time of the application server $R_{a} \cdot \operatorname{MARE}\left(R_{a}\right)$ was calculated as 0.000012 . The small difference between the measured and predicted user response time indicates that tracking filter works pretty well to estimate the model 
parameters in this system. The fourth curve is the tracked service demand of the application server $D_{a}$. The tracked $D_{a}$ decreases a little bit when $\mathrm{N}$ increases. However the difference is not big with different number of clients. It could be considered as a constant as an approximation. The ideal value of $D_{a}$ under different workload is that it keeps that same value. The tracked $D_{a}$ in Figure 7.3 indicates that it is quite good to model the application server as a queueing node. The feature that the service demand is a little bit large when workload is high does not hurt the server provisioning. It implies over provisioning with high workload. The fifth curve (on the bottem) is the number of the clients $N$ in the system. It increases from 2 to 30 , keeps as 30 for some time and then decreases from 30 to 2 .

Overall it is quite satisfactory to use the performance model shown in Figure 7.2 to model the system shown in Figure 7.1.

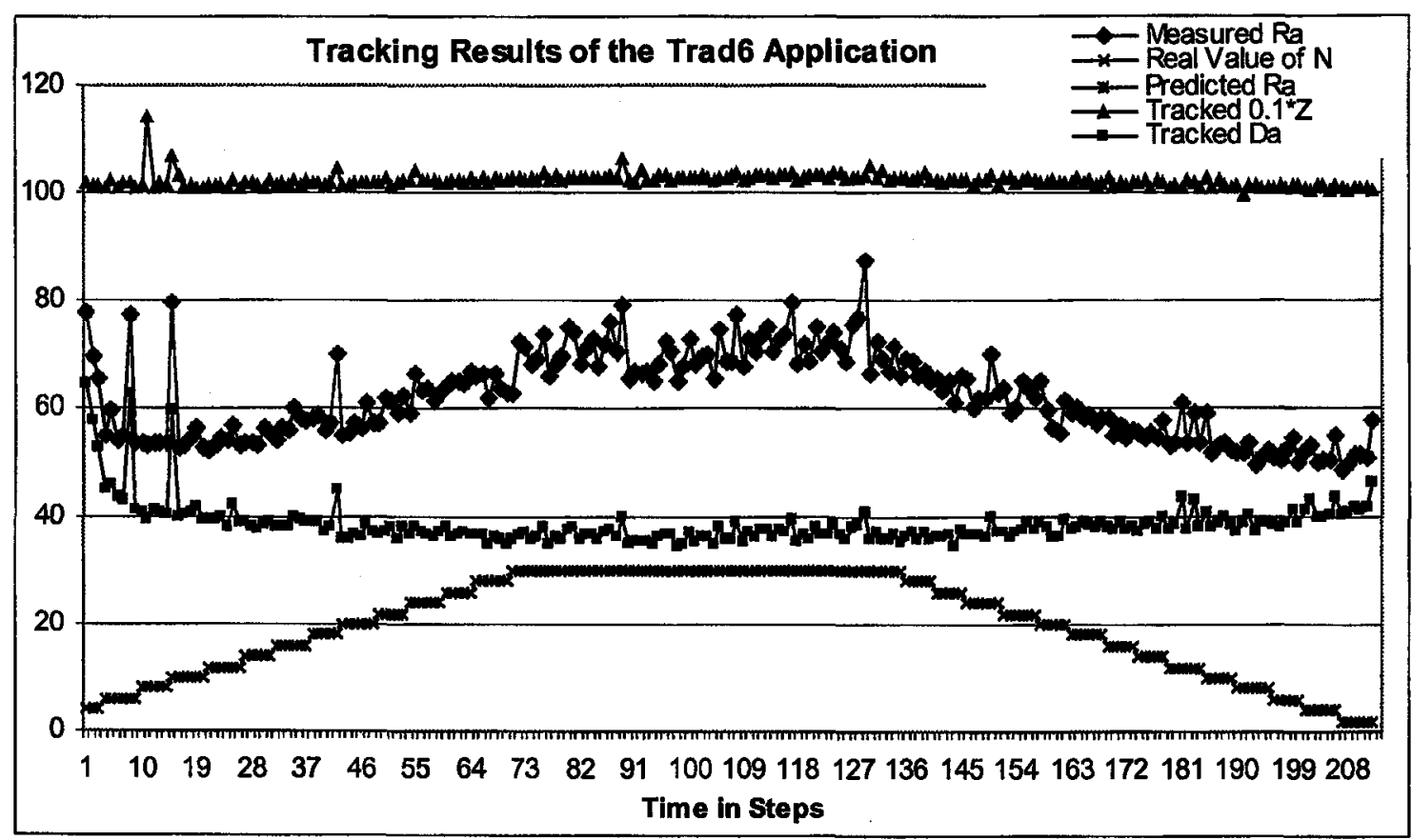

Figure 7.3 Tracking with Varying $N$ ( $N$ is Known to the Filter)

\subsection{Workload representation}

In real systems, both think time $Z$ and number of clients $N$ are difficult to measure dynamically. Most of the time, only the arrival rate is available for measurement. In this 
section, we are going to explore if it is possible to track $Z$ and $N$ at the same time and how to solve this problem if that is impossible.

\section{Track both $N$ and $Z$}

In this section, the tracking parameter vector was $\mathbf{x}=\left[Z, D_{a}, D_{d b}, N\right]$ which includes the number of clients $N$ in addition to the user think time $Z$ and service demands of AppServer and Database. Figure 7.4 shows that the tracked $N$ and $Z$ were far away from the real values. During the first several steps, the tracked number of clients $N$ (the thickest line) was around 1 and the tracked user think time $Z$ was around $600 \mathrm{~ms}$. Then, tracked $Z$ dropped to a value close to 0 and keeps that value at later time. Tracked $N$ became a small value as well and it increased as the workload (real number of users) increased. The actual user think time $Z$ was $1000 \mathrm{~ms}$ and $N$ was between 2 and 30 .

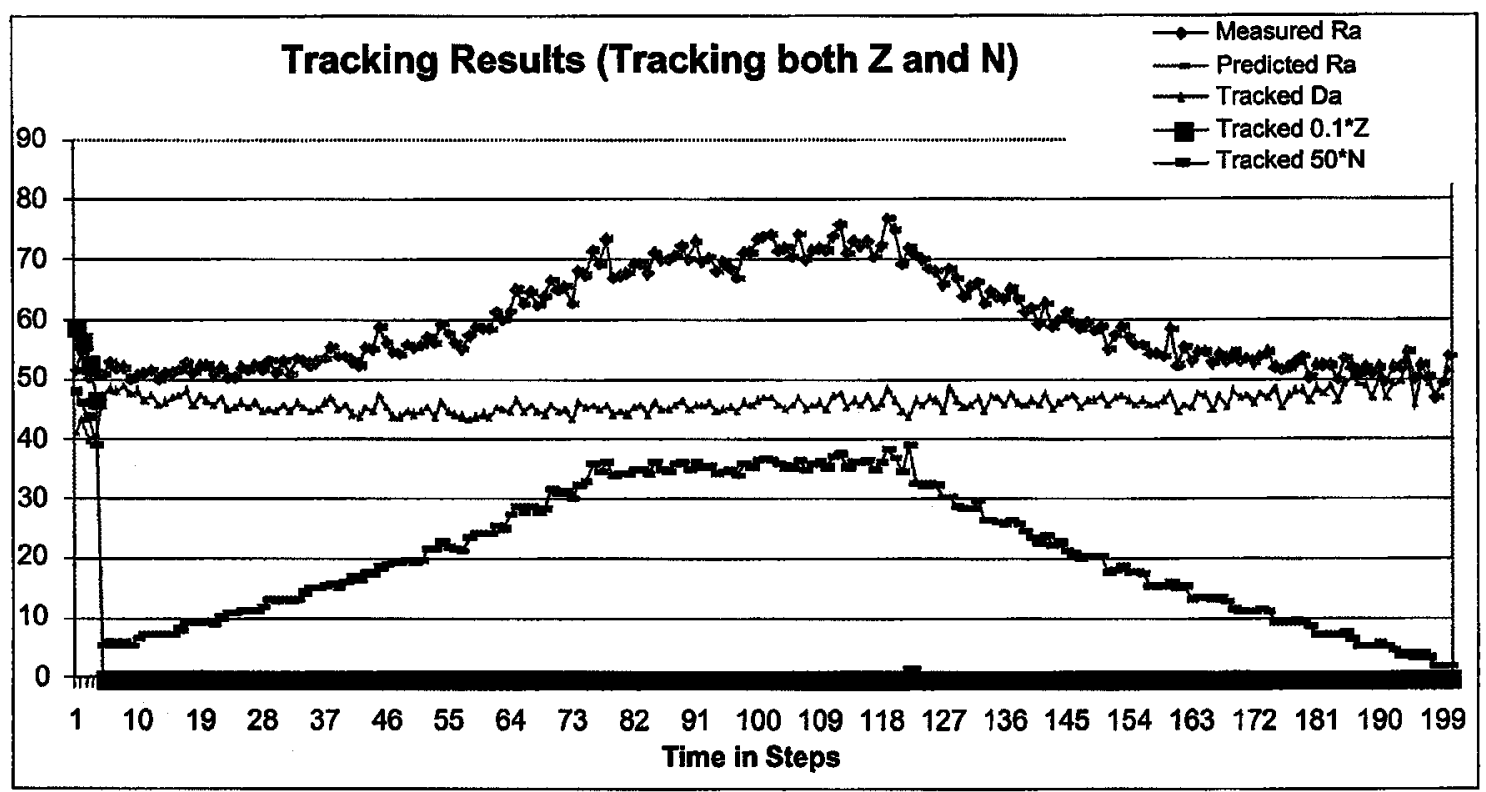

Figure 7.4 Tracking results of Trade 6 Application (Tracking both $Z$ and $N$ )

The other parameters and measurements were similar to Figure 7.3. The measured and predicted response times of application server (the top 2 curves that are almost the same in Figure 7.3) were fitted very well and $M A R E\left(R_{a}\right)$ was 0.00016 . The tracked service demand of the application server $D_{a}$ did not vary a lot (in the range between $40-50 \mathrm{~ms}$ ). It showed that the model was still quite good at predicting the performance although it was not able to track $N$ and $Z$ around the exact values. 


\section{Track $Z$ with fixed $N$}

$N$ and $Z$ are used to represent the client workload in queueing theory for closed queueing network systems. For open queueing network model, the client workload is represented by the arrival rate $\lambda$ only. Analytic solvers based on queueing theory are not able to deal with an open queueing network model if the arrival rate is larger than the service rate. One way to solve this problem is to convert the open queueing model to a closed queueing model using a pair of $N$ and $Z$ using Little's Law. In this case, the analytic solver is able to deal with both open and closed queue network models.

According to Little's Law, $N=\lambda(R+Z)$ where $\lambda$ is the throughput and $R$ is the response time. However, there are not any other equations that establish a relationship between $N$ and $Z$ except Little's Law. For one equation with 2 unknown parameters, there are unlimited solutions. That means it is impossible to track the exact $N$ and $Z$ at the same time. And there are infinite solutions that work as well as the exact values. We must fix one of $N$ or $Z$ to estimate the workload even if it is far away from the real values. In real systems, both $Z$ and $N$ are difficult to measure. Usually it is only possible to use arrival rate to measure the workload. The Kalman filter assumes all the tracking parameters are real values while the number of clients $N$ is supposed to be an integer. It is more convenient to fix $N^{\prime}$ and estimate $Z^{\prime}$.

In order to make the filter work properly, the fixed $N^{\prime}$ value must be big enough to deal with the heaviest workload. For open queueing network, $N^{\prime}>=10 \lambda_{\max } R_{\max }$ where $\lambda_{\max }$ and $R_{\max }$ are the arrival rate and response time under heaviest workload condition. For closed queueing network model, $N^{\prime}>N$. where $\mathrm{N}$ is the actual number of the users in the system. If there is no available knowledge for $N, N^{\prime}>=10 \lambda_{\max } R_{\max }$ can be used in closed queueing network model as well.

Figure 7.5 shows the tracking result with fixed $N^{\prime}\left(N^{\prime}=30\right)$ while measurement $\operatorname{set} \mathbf{z}=$ $\left[R_{a}, R_{d b}, \lambda\right]$. All the measurements and tracked parameters were similar to Figure 7.3 and Figure 7.4 except the tracked user think time $Z$ '. The measured and predicted response times are very close (the top 2 curves in Figure 7.5). $\operatorname{MARE}\left(R_{a}\right)$ was 0.00023 The tracked service demand $D_{a}$ was around $40 \mathrm{~ms}$. On the other hand, the tracking $Z^{\prime}$ varied from $1000 \mathrm{~ms}$ to $10000 \mathrm{~ms}$. Its shape was like a bowl in Figure 7.5. When $N^{\prime}$ was different 
from the actual value, the tracked user think time $Z$ ' was adjusted to fit the measurements. When the fixed $N^{\prime}$ was larger than the actual value, the tracked $Z^{\prime}$ is larger than the actual value as well. When the actual number of clients became 30 , the tracked user think time converged to around $1020 \mathrm{~ms}$ which was close to the actual value $1000 \mathrm{~ms}$.

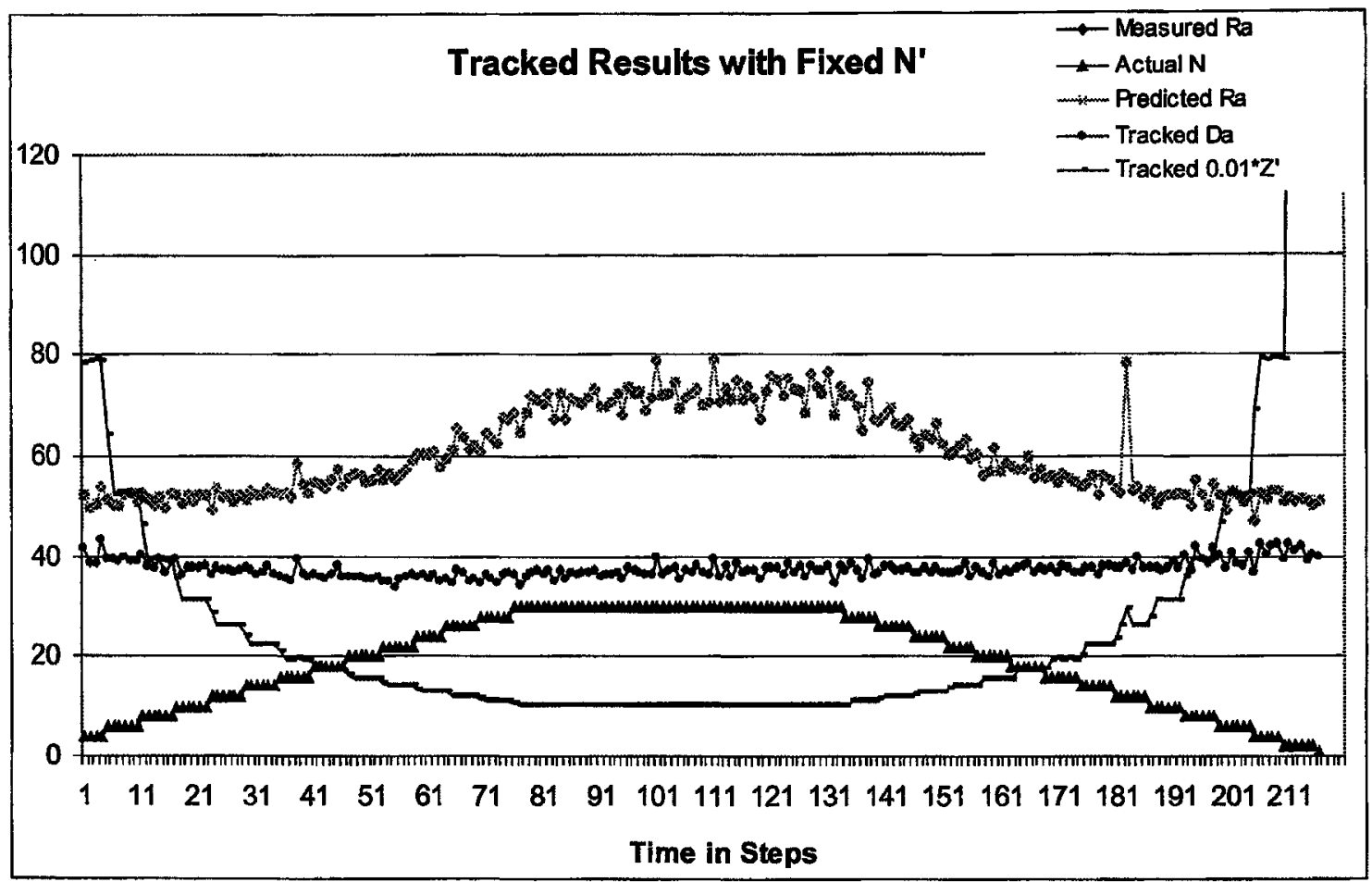

Figure 7.5 Tracking Result with Fixed $N^{\prime}\left(N^{\prime}=30\right)$

\subsection{Server provisioning}

This section implements controlled server provisioning to the system described in Figure 7.1. A workload predictor was incorporated; it implemented a linear regression algorithm based on the method of least squares and forecasts the system throughput for the next sampling period. Provisioning decisions were made based on the forecasted performance with the calibrated performance model and the forecasted throughput. 


\section{Workload Predictor with Regression Algorithm}

The general form of a simple linear regression is

$$
Y_{i}=a X_{i}+b
$$

where $a$ is the slope and $b$ is the intercept. $X_{i}$ and $Y_{i}$ are the collected data from the sample or population, and $a$ and $b$ are the unknown parameters to be estimated from the $x_{i}$ and $y_{i}$. The estimates for the values of $a$ and $b$ derived by least squares [9] are shown

$$
\hat{a}=\frac{\sum\left(x_{i}-\bar{x}\right)\left(y_{i}-\bar{y}\right)}{\sum\left(x_{i}-\bar{x}\right)^{2}}
$$

and

$$
\hat{b}=\bar{y}-\hat{a} \bar{x}
$$

where $\bar{x}$ is the mean of the $x$ values and $\bar{y}$ is the mean of the $y$ values. $\hat{a}$ and $\hat{b}$ are the estimates of $a$ and $b$.

The workload predictor collected the measured workload $(R$ or $\lambda)$ as $y$ and considered the measurement step sequence number as $x$. The predicted workload $y_{k}$ at measurement step $k$ can be derived as:

$$
y_{k}=\hat{a} x_{k}+\hat{b}
$$

\section{Filter Out the Spikes}

In real systems, the monitored parameter (e.g. response time) related to the SLA may have spikes for unknown reason from time to time. The spikes may lead to wrong provisioning decisions. In order to avoid the wrong decisions due to the spikes, a small slide-moving window with 3 measurement steps was built to filter out the spikes. The decisions base on the 3 values in the slide-window. A new decision will be made only when at least two values agree. This mechanism ignores the less frequent spikes.

\subsubsection{Server provisioning with zero delay}

For simplicity, it is assumed that the provisioning was finished immediately in this case. All the server replicas are standby if they are not in use. They are added to or removed from the cluster immediately. 


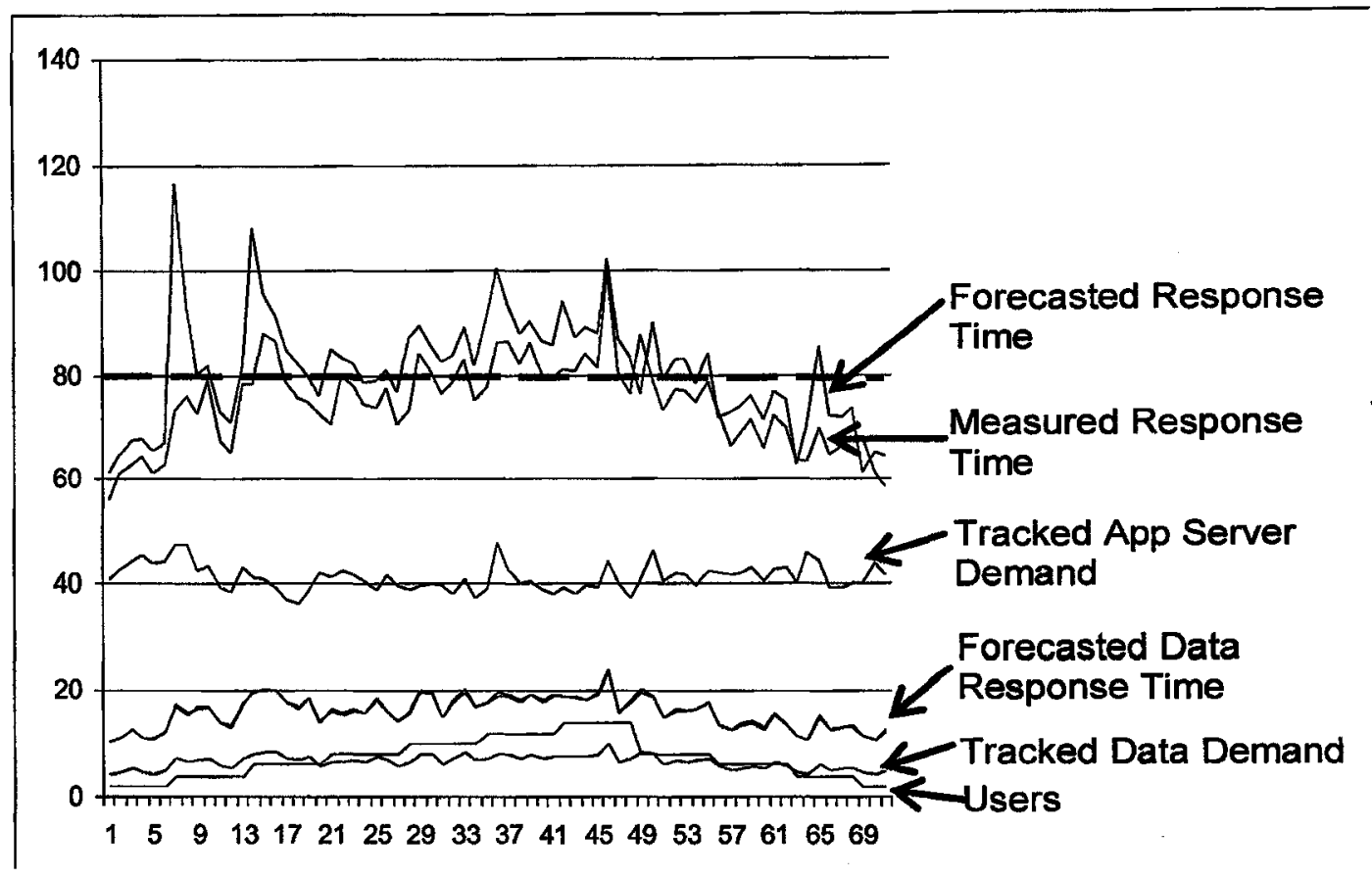

Figure 7.6 Tracking and Forecasting with No Provisioning (zero delay)

Figure 7.6 shows the results (time is in milliseconds) obtained with no provisioning. The results are shown for 70 sample periods, that is 35 minutes. The two metrics tracked by the Kalman filter are Tracked App Server Demand and Tracked Data Demand. They are the model parameters $S_{a}$ and $S_{d}$ shown in Figure 7.2. Measured Response Time and Forecasted Response Time are the response times monitored and computed by the performance model for the AppServer. They have an average error around 9.5\%. Since there is no provisioning, the SLA threshold $(80 \mathrm{~ms})$ is surpassed frequently by the Measured Response Time. 


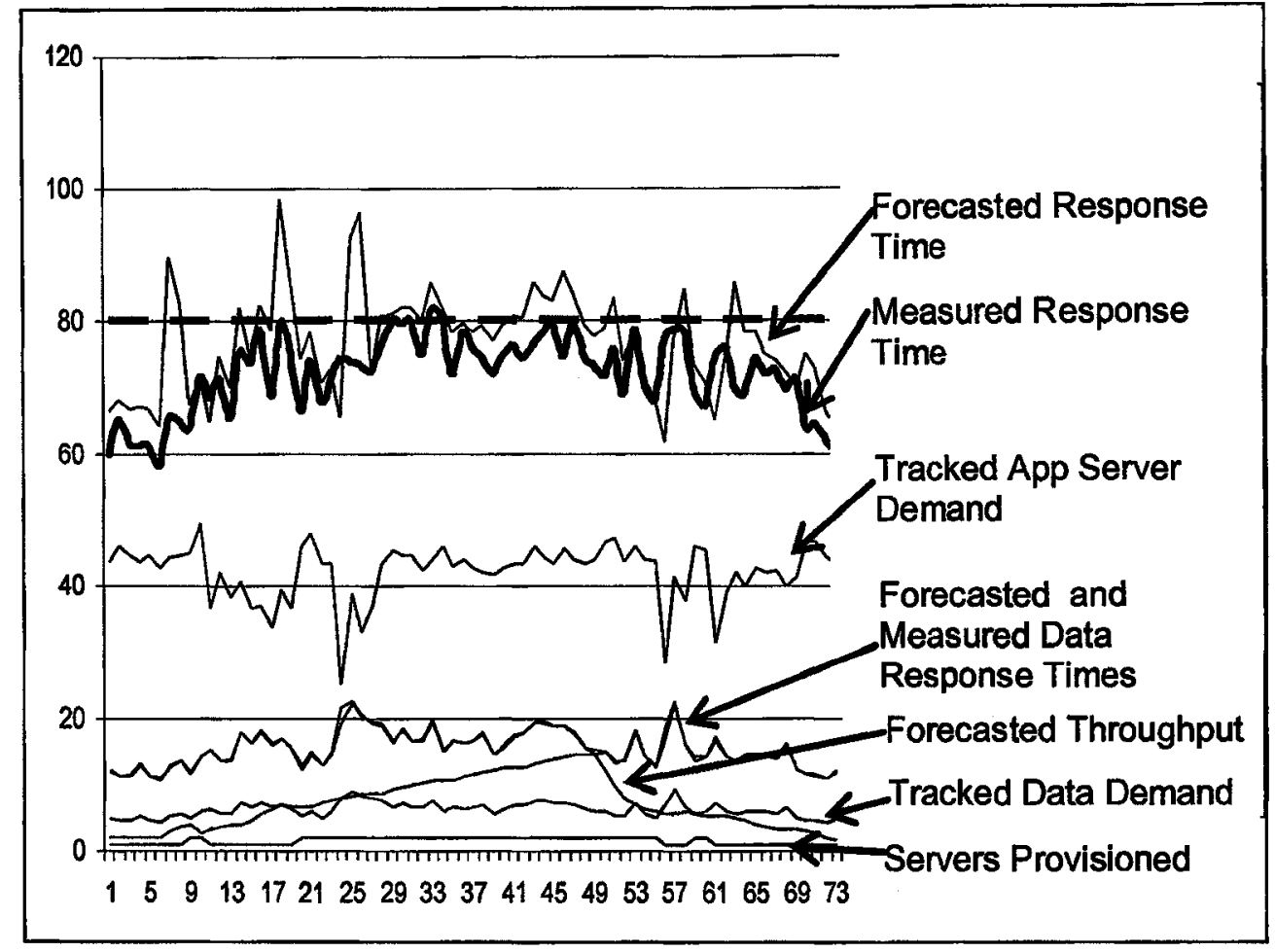

Figure 7.7 Tracking, Forecasting and Provisioning (zero delay)

Figure 7.7 shows the same experiment, but with the provisioning enabled. There are 2 new metrics shown in this figure: the Forecasted Throughput, meaning the system throughput predicted by the regression algorithm for the next sampling period, and the Servers Provisioned, meaning the application servers in the cluster at any given time. Whenever the Forecasted Response Time exceeds the SLA objective, a new server was added. If it is possible to meet the SLA objectives with a smaller number of servers, one server was removed to save the resource cost. As a result, Servers Provisioned alternated between 1 and 2 and provided enough capacity to handle the load and to meet the SLA objective. Adding and removing the servers to the cluster implies changes in the structure and parameters of the performance model as well. As a result, there may be transients in tracking the system parameters. The measured response times were mostly less than $80 \mathrm{~ms}$ with a few exceptions.

In this case, the time of adding / removing servers was 0 . As all the servers are on all the time. The workload generator distributed the requests to the server directly according to the number of servers need. 


\subsubsection{Server provisioning with provisioning delay}

The system shown in Figure 7.1 uses IBM Tivoli Provisioning Manager (TPM).to execute the provisioning physically. When a new server replica is needed to meet the SLA, TPM will execute workflow scripts to add a server replica to the cluster. If fewer server replicas can meet the SLA, TPM will execute workflow scripts to remove a server replica from the cluster as well. When TPM is involved in server provisioning, the provisioning time is not zero anymore. Provisioning delay, the time between making a provisioning decision and provisioning actually completed, can not be ignored anymore. During the experiments, the sampling period was 30s. The time of adding and removing servers were around 5-10 $\mathrm{min}$ and 1-2 min respectively. The provisioning delay of adding / removing servers is 10-20 / 2- 4 measurement steps.

In this section, the SLA is that the measured response time of the application server $R_{a}$ $<=115 \mathrm{~ms}$. The workload change pattern is similar to the one described in section 7.2. The number of users $N$ increases from 2 to 30 , stays as 30 for some time and then decreases from 30 to 2 . The think time $Z$ is set to $1000 \mathrm{~ms}$. However the filter does not know anything about $N$ and $Z$. It uses a fixed $N^{\prime}=1000$ to track the workload.

\section{Tracking Results with No Provisioning}

Figure 7.8 shows the measured response times and throughput with 1 server in the cluster (no provisioning). The measured response time could be as big as $400 \mathrm{~ms}$. The $\operatorname{SLA}\left(R_{a}<=115 \mathrm{~ms}\right)$ was violated when the when the workload was over a threshold.

\section{Reactive Provisioning without Considering Provisioning Delay}

The control used in this section is the same as the control used in section 7.4.1. Whenever the measured response time was more than 115ms (SLA_High), the autonomic manager controlled the TPM to add servers. When the measured response time is less than 70ms (SLA_Low), the autonomic manager tries to check if it is able to remove servers to meet SLA with less resource. Figure 7.9 shows the tracking results with reactive provisioning without considering the provisioning delay. The SLA (i. e. 115ms) were violated during the provisioning delay. 


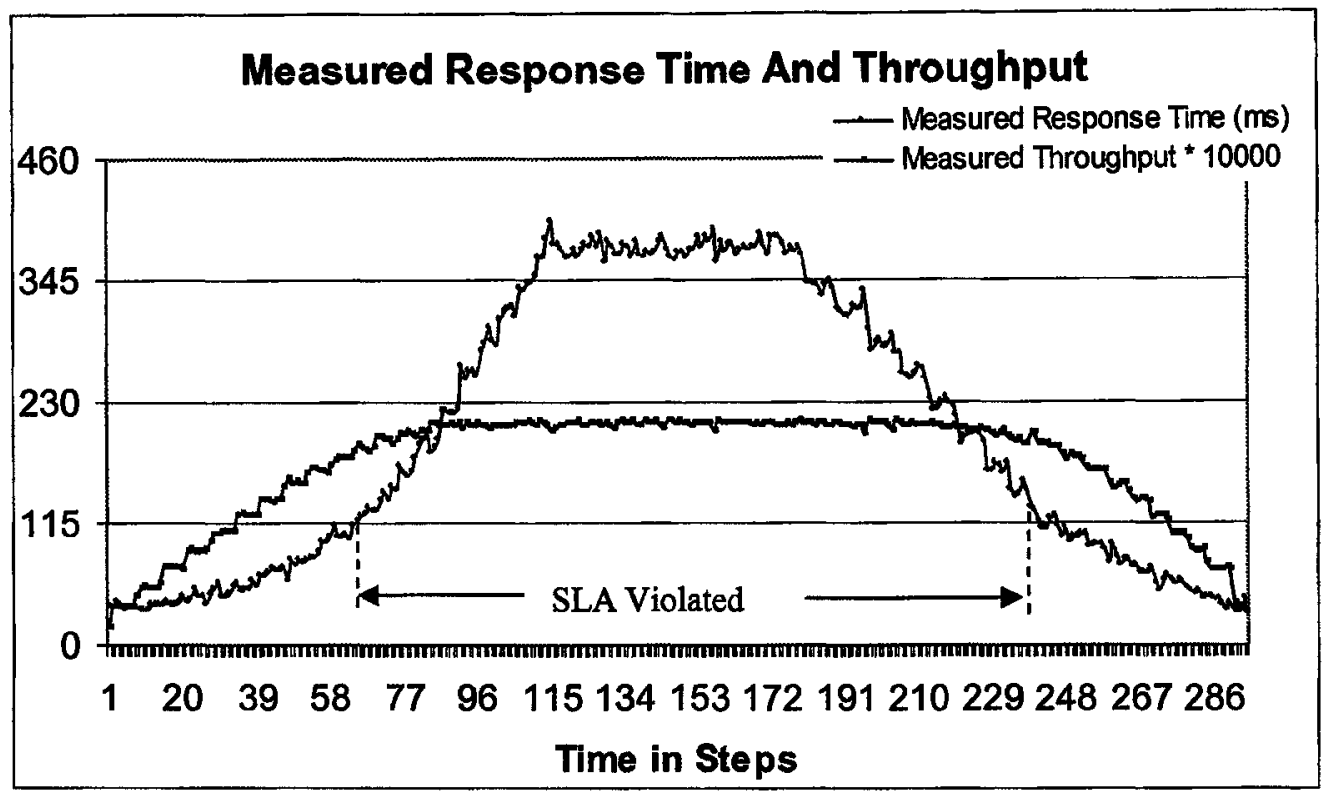

Figure 7.8 Tracking Result with No Provisioning

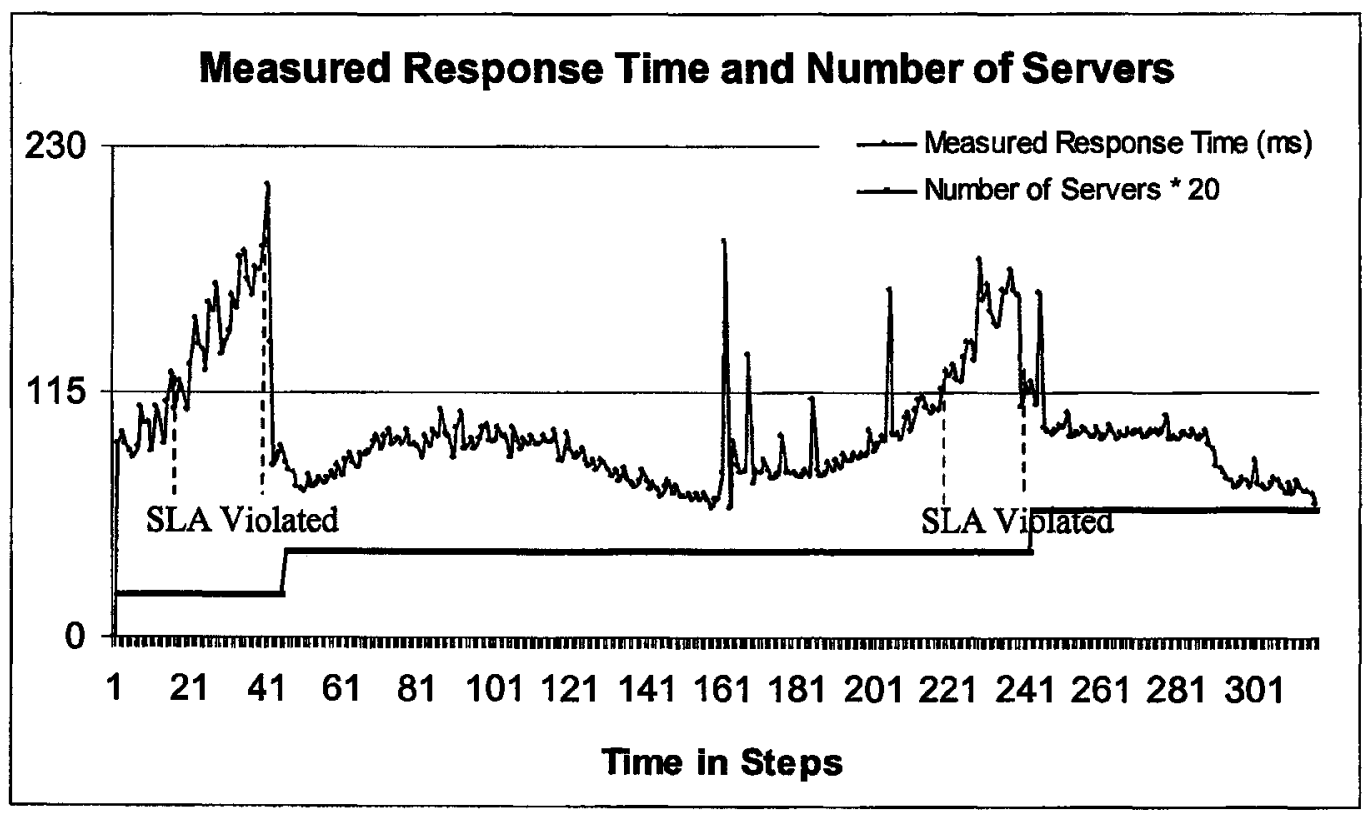

Figure 7.9 Tracking Results with Reactive Provisioning without Considering Provisioning Delay 


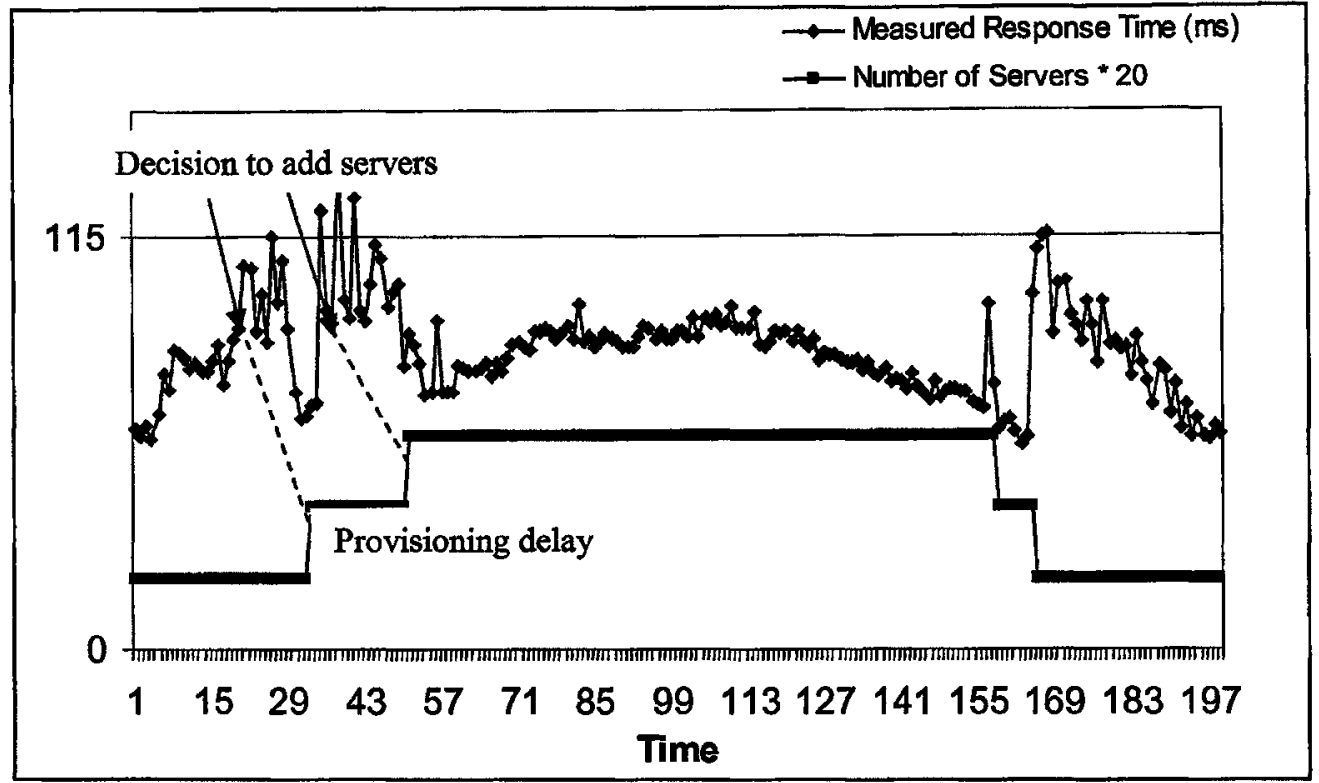

Figure 7.10 Tracking Results with Predictive Provisioning Control

\section{Predictive Provisioning Control}

In order to solve the problem of provisioning delay, a workload predictor based on a linear regression algorithm was implemented. It estimated $a$ and $b$ from the recent workload data. As mentioned before, the provisioning delay of adding and removing servers are quite different. The Decision Maker first checked $a$. If $a>0$, it used the provisioning delay of adding servers to predict how many new servers are needed. If $a<$ 0 , it used the provisioning delay of removing servers to predict how many new servers could be removed in the future. Figure 7.10 shows the tracking trace with predictive provisioning control. The measured response times are no more than $115 \mathrm{~ms}$ unless a few spikes. 


\section{CHAPTER 8 CONCLUSIONS}

This thesis proposed a framework for automated dynamic resource management. It applied the layered queueing network (LQN) model and tracking filter to the feedback control to optimize the system configuration according to the service level agreements (SLA). Although the target system in this thesis is the multi tier information system such as the J2EE application system, the framework could be used to many other dynamic systems as well.

The tracking filter works well to estimate the system parameters of the queue-based performance models. The experiment results showed that the iterated extended Kalman filter (IEKF) is better than the extended Kalman filter (EKF). The experiment results showed that if the measurement step size $T$ is chosen properly and the coefficients of variation of the tracking parameters are no more than 1, the MAREs (mean absolute relative errors) are usually no more than 0.1 and fMAREs (forward-looking MAREs) are less than 0.15 .

Because of the prediction errors in tracking filter, it is better to use a guard band for the threshold of SLA for provisioning control or admission control. The guard band helps to avoid the dithering in resource management. In the experiments on a real case study an additional smoothing in the decision process was added, by requiring several steps to exceed the threshold, to trigger a decision.

The tracking quality of the filter depends on the filter parameters setting. Some suggestions of choices of the filter parameters, such as measurement step time $T, \mathbf{Q}$ and $\mathbf{R}$ matrices, are provided after evaluating the tracking quality with different settings. The experimental results suggested to use a simulation to determine the standard measurement step time $T^{*}$ first, then choose $T$ between $0.1 T^{*}$ and $T^{*}$ considering the change rate the system parameters. $\mathbf{Q}$ and $\mathbf{R}$ matrices could be diagonal matrices with fixed values for simplicity. The $\mathbf{R}$ matrix can be adaptive to achieve better tracking quality as shown in section 5.6. Even in systems with more than 1 class of users and more than 1 entry in a task, the forward-looking mean absolute relative error (fMARE) to guide 
system configuration is generally no more than 0.15 .

The hill climbing algorithm works well with performance model and tracking filter for dynamic resource management. It is able to add/remove a number of server replicas at a time if necessary while the control methods with no model do not have that ability.

The framework has been applied to a real system with Trade 6 benchmark application as well. By using a big fixed number user $N$ in the filter and the related estimated user think time $Z$, the tracking filter is able to simulate both the open and closed arrivals pretty well. A workload predictor helps to solve the problem of provisioning delay.

There are some limitations in the filtering approach to parameter tracking. (1) If the system has a lot of parameters to track, the tracking quality may suffer (as shown in section 5.7). (2) The analytic solver uses a fixed point iteration which sometimes has convergence problems (for example if the model is saturated or has many replicas). The time to solve a model may be too long for practical use.

\section{Future work}

The performance model and tracking filter have been used predict the performance metrics of application server quite accurately. However, the performance prediction for database server is not good enough. The effect of using other resources (e.g. caches, buffers, storages etc.) and locks make the database server a complex subsystem. A simple queueing node is not accurate enough to represent the database server. A more detailed performance model must be studied to represent the behavior of database server.

This research has not studied the assignment of multiple applications to one processor with different shares. In virtualized systems, a number of applications may share the same processor even though all the users seem to use the whole processor. The LQN model is able to assign a speed rate to a processor. This problem could be solved with careful considerations.

The Decision Maker in the proposed framework used the hill climbing algorithm for resource management. There might be other optimization or search methods which can solve the same problem better. 


\section{References:}

[1] M. Abdeen, and C. M. Woodside, "Seeking Optimal Policies for Adaptive Distributed Computer Systems with Multiple Controls". Proc. Third International Conference on Parallel and Distributed Computing, Applications and Technologies (PDCAT'02), Kanazawa, Japan, Sept. 2002

[2] T. F. Abdelzaher and N. Bhatti, "Web server QoS management by adaptive content delivery", International Workshop on Quality of Service, London, UK, June 1999

[3] T. Abdelzaher, K. G. Shin and N. Bhatti, "Performance Guarantees for Web Server End-Systems: A Control-Theoretical Approach", IEEE Transactions on Parallel and Distributed Systems, Vol. 13, No. 1, Jan 2002

[4] A.O. Allen, Probability, Statistics and Queueing Theory with Computer Science Applications, 2nd ed., Academic Press, 1990.

[5] Åström, K.J. and Wittenmark B. Adaptive Control. 2nd edition. Addison-Wesley Publ Co, 1995

[6] G. Bolch, S. Greiner, H. de Meer, K. S. Trivedi, Queueing Newworks and Markov Chains, John Wiley \& Sons, 1998

[7] E. Brookner, Tracking and Kalman Filtering Made Easy, Wiley Interscience, 1998

[8] J. Chen, G. Soundararajan, C.Amza, "Autonomic Provisioning of Backend Databases in Dynamic Content Web Servers", Proceedings of the Third International Conference on Autonomic Computing, Dublin, June 2006

[9] N. R. Draper, H. Smith, Applied Regression Analysis, Wiley Series in Probability and Statistics, 1998

[10] Y. Diao, X. Lui, S. Froehlich, J. L. Hellerstein, S. Parekh, L. Sha, "On-Line Response Time Optimization of An Apache Web Server", International Workshop on Quality of Service, 2003

[11] H.M. El-Sayed, D. Cameron, and C.M. Woodside, "Automated performance modeling from scenarios and SDL designs of distributed systems", Proc. of the Int. Symposium on Software Engineering for Parallel and Distributed Systems (PDSE'98), Kyoto, April 1998

[12] H. M. El-Sayed “A Framework For Automated Performance Engineering of Distributed Real-Time Systems", Thesis of Doctor of Philosophy. Ottawa-Carleton 102 
Institute for Electrical Engineering, Faculty of Engineering, Department of Systems and Computer Engineering Carleton University 1999

[13] L. J. N. Franken, and B. R. Haverkort, "Reconfiguring Distributed Systems using Markov-Decision Models", Proc. Trends in Distributed Systems (TreDS'96), Oct. 1996, pp. 219-228

[14] G. Franks, A. Hubbard, S. Majumdar, D. Petriu, J. Rolia, and C.M. Woodside, “A toolset for performance engineering and software design of client-server systems", Performance Evaluation, 24 (1-2):117-135, November 1995.

[15] G. Franks, S. Majumdar, J.E. Neilson, D.C. Petriu, J.A. Rolia and C.M. Woodside, "Performance Analysis of Distributed Server Systems", Proc. Sixth International Conference on Software Quality, Ottawa, Canada, October 28-30, 1996, pp. 15-26

[16] G. Franks, "Performance Analysis of Distributed Server Systems," PhD. thesis, Carleton University, Jan. 2000.

[17] N. Gandhi, J. L. Hellerstein, S. Parekh, and D. M. Tilbury, "Managing the Performance of Lotus Notes: A Control Theoretic Approach," Proceedings of the Computer Measurement Group, 2001

[18] J. Hellerstein, Y. Diao, S. Parech, D. Tilbury, Feedback Control of Computing Systems, John Wiley \&Sons, Inc., 2004

[19] A. H. Jazwinski, Stochastic Processes and Filtering Theory. New York: Academic Press, 1970.

[20] IBM, "An architectural blueprint for autonomic computing", IBM and Autonomic Computing, June, 2005

[21] IBM Tivoli Intelligent Orchestrator, http://www306.ibm.com/software/tivoli/products/intell-orch/, Jan 23, 2005

[22] R. Jain, The Art of Computer Systems Performance Analysis. Wiley, 1991

[23] S. Julier, J. Uhlmann, H. F. Durant-Whyte, "A new method for approximating nonlinear transformations of means and covariances in filters and estimators," IEEE Transactions on Automatic Control, vol. 45, no. 3, pp. 477-482, March 2000

[24] R.E. Kalman, "A new approach to linear filtering and prediction problems", Transactions of ASME, Journal of Basic Engineering, vol. 82, pp 34-45, March 1960

[25] E. Kandogan, C. S. Campbell, P. Khooshabeh, J. Bailey, P.P. Maglio, "Policy- 
based Management of an E-commerce Business Simulation: An Experimental Study", Proceedings of the Third International Conference on Autonomic Computing, Dublin, June 2006

[26] S. Keshav, "A Control-Theoretic Approach to Flow Control", ACM SIGCOMM Computer Communication Review, Jan. 1995

[27] T. Lefebvre, H. Bruyninckx, and J. De Schutter, "Kalman filters for nonlinear systems: a comparison of performance," Internal Report 01R033, KU Leuven, 2001

[28] M. Litoiu, and J. Rolia, "Object Allocation for Distributed Applications with Complex Workloads", Lecture Note in Computer Science 1786, Springer, 2000, pp 25-39

[29] M. Litoiu, C. M. Woodside, T. Zheng, "Hierarchical model based autonomic control of software systems," Proceedings of Design and Evolution of Autonomic Software (DEAS'05) Workshop, St. Louis, USA, May 2005

[30] C.L. Liu and J.W. Layland, "Scheduling algorithms for multiprogramming in a hard real-time environment", Journal of the Assoc. Computing Mach., vol. 20(1), pp. 46-61, 1973.

[31] Z. Liu, L. Wynter, C. H. Xia, F. Zhang, "Parameter inference of queueing models for IT systems using end-to-end measurements", Performance Evaluation, volume 63, issue 1 , Jan. 2006, pp36-60

[32] Y. Lu, T. Abdelzaher, C. Lu, L. Sha, X. Liu, "Feedback Control with QueueingTheoretic Prediction for Relative Delay Guarantees in Web Servers", Real-Time and Embedded Technology and Applications Symposium, Toronto, Canada, May 2003

[33] D. A. Menasce, M. Bennani, "On the Use of Performance Models to Design SelfManaging Computer Systems", Proc. 2003 Computer Measurement Group Conference, Dallas, TX, Dec. 7-12, 2003

[34] D. A. Menasce, "QoS-aware software components", IEEE Internet Computing, March/April 2004, Vol. 8, No. 2

[35] A. Moghaddamjoo, R. L. Kirlin, "Robust adaptive Kalman filtering with unknown inputs", IEEE Trans. Acoust. Speech Signal Process. 37 (8) (August 1989) 11661175.".

[36] J. E. Neilson, C. M. Woodside, D. C. Petriu, and S. Majumdar, "Software 104 
Bottlenecking in Client-Server Systems and Rendez-vous Networks". IEEE Trans. On Software Engineering. Vol. 21, No. 9, September 1995, pp. 776-782

[37] M. Norgaard, N.K Poulsen, and O. Ravn, "New developments in state estimations for nonlinear systems", Automatica, vol 36, no. 11, pp. 1627-1638, November 2000

[38] The Open Group, "ARM 4.0 Java Language Binding Technical Standard 4.0", Oct. 2003

[39] D. Petriu, C. M. Woodside, "Analysing Software Requirements Specifications for Performance", Report SCE-02-02, Dept of Systems and Computer Engineering, submitted for publication, Feb. 2002

[40] M. L. Puterman, Markov Decision Processes, Discrete Stochastic Dynamic Programming, Wiley Interscience, 2005

[41] V. J. Rayward-Smith, I. H. Osman, C. R. Reeves, Modern Heuristic Search Methods, John Wiley \& Sons. December 1996

[42] S. Redana and A. Capone, "Received power based call admission control techniques for UMTS uplink", Proceedings of Vehicular Technology Conference, Vol. 4, pp. 2206-2210, Sept. 2002.

[43] M. Reiser and S. Lavenberg, "Mean value analysis of closed multichain queueing networks", J. ACM, vol. 27, no. 2, Apr. 1980.

[44] J. R. Rolia and Kenneth Sevcik, "The method of layers", IEEE Transactions on Software Engineering, Vol. 21, No. 8, pp. 689-700, 1995.

[45] M.-V. M. Salem, G. Bochmann, J. W. Wong, " Server Selection for Differentiated classes of users", Int. Symp. on Performance Evaluation of Computer and Telecommunication System, San Diego, July 2002

[46] F. E. Schneider and D. Wildermuth, "Using an extended Kalman filter for relative localisation in a moving robot formation", Proc. 4th Int. Workshop on Robot Motion and Control, Wachtberg, Germany, 2004, pp. 85- 90

[47] K. G. Shin, C. M. Krishna, and Y-H. Lee, "Optimal Dynamic Control of Resources in a Distributed System", IEEE Transactions on Software Engineering. Vol. 15, No. 10, October 198

[48] C.U. Smith, Performance Engineering of Software Systems, Addison-Wesley Publishing Co., New York, NY, 1990. 
[49] L. Stojanovic, J. Schneider, A. Maedche, S. Libischer, R. Studer, Th. Lumpp, A. Abecker, G. Breiter, and J. Dinger, "The role of ontologies in autonomic computing systems," IBM Systems Journal, v. 43, n. 3, 2004

[50] R. S. Sutton, A. G. Barto, Reinforcement Learning: an Introduction, Cambridge, MA: MIT Press, 1998

[51] G. Tesauro, "Online resource allocation using decompositional reinforcement learning", Proceedings of the AAAI-05, 2005

[52] G. Tesauro, N. K. Jong, R. Das, M. N. Bennani, "A Hybrid Reinforcement Learning Approach to Autonomic Resource Allocation", Proceedings of the Third International Conference on Autonomic Computing, Dublin, June 2006

[53] H. Tanizaki, Nonlinear Filters: Estimation and Applications-Second, Revised and Enlarged Edition, Springer-Verlag, Berlin-Heilderberg, 1996

[54] B. Urgaonkar, G. Pacifici, P. Shenoy, M. Spreitzer, A. Tantawi, "An analytical model for multi-tier internet services and its applications", Proceedings of SIGMETRICS-05, 2005

[55] D. Vengerov, N. Iakovlev, "A reinforcement learning framework for dynamic resource allocation: First results", Proceedings of ICAC-05, 2005

[56] Hai Wang , K.C. Sevcik, "Experiments with improved approximate mean value analysis algorithms", Performance Evaluation, v.39, p.189-206, Feb. 2000

[57] G. Welch, G. Bishop, "An Introduction to the Kalman Filter", TR 95-041, Department of Computer Science, University of North Carolina

[58] C.M. Woodside, "Throughput calculation for basic stochastic rendezvous networks", Performance Evaluation, Vol. 9, No. 2, pp. 143-160, 1989.

[59] C. M. Woodside, J. E. Neilson, D. C. Petriu, and S. Majumdar, "The stochastic rendezvous network model for performance of synchronous client server-like distributed software", IEEE Transactions on Computers, 44(1):20-34, January 1995.

[60] C. M. Woodside, T. Zheng, M. Litoiu , "The Use of Optimal Filters to Track Parameters of Performance Models", Proceedings of 2nd International Conference on Quantitative Evaluation of Systems (QEST05), Torino, Sept. 2005.

[61] C. M. Woodside, T. Zheng, M. Litoiu, "Service System Resource Management Based on a Tracked Layeres Performance Model", Proceedings of the Third 106 
International Conference on Autonomic Computing, Dublin, June 2006

[62] Q. Zhang, L. Cherkasova and E. Smirni, "A Regression-Based Analytic Model for Dynamic Resource Provisioning of Multi-Tier Applications", Proceedings of the Fourth International Conference on Autonomic Computing, Jacksonvilla, June 2007

[63] T. Zheng, "Optimization of Distributed Real-Time Systems with Scenario Deadlines", M. Eng thesis, Carleton University Aug 2002

[64] T. Zheng, C. M. Woodside, "Heuristic Optimization of Scheduling and Allocation for Distributed Systems with Soft Deadlines", Proceedings of 13th International Conference on Computer Performance Evaluation, Modelling Techniques and Tools (TOOLS 2003), Urbana, Illinois, USA, Sept 2003, pp 169-181, vol. LNCS 2794, Lecture Notes in Computer Science, Springer-Verlag, 2003

[65] T. Zheng, J. Yang, C. M. Woodside, M. Litoiu, G. Iszlai, "Tracking Time-Varying Parameters in Software Systems with Extended Kalman Filters", Proceedings of CASCON 2005, Toronto, Oct. 2005

[66] W. B. Zhu, "Simulation Generator for Layered Queueing Networks", RADS report

[67] http://www.opengroup.org/management/arm.htm/

[68] http:/www.markwilson.co.uk/blog/2005/10/microsofts-dynamic-systemsinitiative.htm

[69] Trade 6 Benchmark, http://www-128.ibm.com/developerworks/edu/dm-dw-dm0506lau.html, Jan 25, 2006

[70] Java EE: Overview. http://java.sun.com/javaee/

[71] http://www.oracle.com/technology/products/jdev/htdocs/jclient/j2EE.GIF 


\title{
Appendix A: Overview of CsimSimulator, CsimTracker and CsimController
}

\begin{abstract}
A.1 Overview
CsimSimulator is an LQN simulator using CSIM 19 simulation engine. It is a $\mathrm{C} / \mathrm{C}++$ program running on windows operating system. CsimSimulator simulates an LQN model, collects and records the statistics of the performance metrics. The statistics could either be written to an output file or sent to another program using socket communication. CsimSimulator is able to modify the LQN parameters at run time if necessary.

CsimTracker is a tracking filter program written in Java. It parses the output file generated by CsimSimulator to get the measurement data and predicts the performance metrics using lqns. (Iterated) Extended Kalman Filter is implemented to estimate the LQN model parameters by comparing the predictions and measurements.

CsimController is a controller program written in Java. It gets the measurement data from CsimSimulator through socket communication. In addition to the tracking filter function, CsimSimulator predicts the performance metrics with the estimated model parameters and compares them with the required SLA. Search algorithms and/or optimization methods are used to suggest a good configuration of the simulated system. The new configuration will be sent to CsimSimulator through sockets as well.
\end{abstract}

\section{A.2 CsimSimulator}

CsimSimulator bases on the LQ-CSIM project proposed by Wenbo Zhu [66] originally. The project used a java program called Parselqn.java and some libraries to generate CSIM simulator code from an LQN model file on UNIX OS. The CSIM simulator includes the programs shown in table A.1. In the LQ-CSIM project, all the model parameters (e.g. service demands, number of threads) are fixed during the whole simulation. 


\begin{tabular}{|c|c|}
\hline Code & Descriptions \\
\hline main.cpp & main control of the whole simulation model \\
\hline bootstrap_csim.cpp & bootstrap the simulation cycle for the model; driver for the reference tasks \\
\hline csim_worker.cpp & generic cross-task call request-message processing routine \\
\hline init_tables.cpp & $\begin{array}{l}\text { initialize the CSIM table for each activity/phase and supports run-length } \\
\text { control }\end{array}$ \\
\hline act_calls_new.cpp & activity_call procedures per activity/phase in all non-reference tasks \\
\hline ref_act_calls_new.cpp & activity_call procedures per activity/phase in all reference tasks \\
\hline output.cpp & $\begin{array}{l}\text { collecting the data, calculating the model performance parameters and } \\
\text { generating the fixed format result file }\end{array}$ \\
\hline $\begin{array}{l}\text { csim_init.cxx } \\
\text { csim_end.cxx }\end{array}$ & customized CSIM control routines \\
\hline csim_init.h & $\begin{array}{l}\text { global CSIM simulation resource initialization, for LQN process/task and } \\
\text { replication information }\end{array}$ \\
\hline sim_para.hxx & simulation control parameters - customizable \\
\hline Iqn.hxx & $\begin{array}{l}\text { data structures for LQN process/task and other global data structure } \\
\text { declarations }\end{array}$ \\
\hline $\begin{array}{l}\text { sync_ref.cpp } \\
\text { sync_count.cpp } \\
\text { sync_event.cpp }\end{array}$ & activities/tasks synchronization support lib routines and classes \\
\hline $\begin{array}{l}\text { act_calls.hxx } \\
\text { ref_act_calls.hxx } \\
\text { csim_workers.hxx } \\
\text { csim_tables.hxx } \\
\text { bootstrap_csim.hxx } \\
\text { sync_*.hxx }\end{array}$ & header files \\
\hline
\end{tabular}

Table A.1 the programs of the CSIM simulator

109 
In order the make the CSIM simulator more flexible, more functions and parameters are added to the generated CSIM simulator (It will be referred as CsimSimulator in the rest of the appendix).

The new functions are:

1. CsimSimulator has a new $\mathrm{C}++$ program called output.cpp which collects, calculates and records the statistics of performance metrics at each measurement step. The output.cpp is able to work in two different modes. In mode 1 , the statistics is written to an output file at each step. In mode 2, the statistics is sent to another program (e.g. CsimController) using socket communication. In mode 2, CsimSimulator is able to change the number of users, threads or replicas according to the commands it received from CsimController. A Boolean variable called writeToFile in sim_para.hxx determines the working mode. If writeToFile is true, CsimSimulator works in mode 1 . Otherwise it works in mode 2. The format of the statistics and communication protocol will be introduced later.

2. In bootstrap_csim.cpp, a new function called change_service_demands is added to determine which service demands should be changed during at certain rate. The pattern of changing the service demand is implemented in getNewSimMean function. Currently, two change patterns are implemented. These methods could be customized for different requirements.

3. In LQ-CSIM, processor_replica[], task_replica[] and task_multiserver[] are used to store the number of replicas and threads. In CsimSimulator, those parameters are defined as the number of replicas and threads allowed to use currently. These parameters could be modified during simulation. New parameters processor_replica_max []$, \quad$ task_replica_max []$\quad$ and task_multiserver_max[] are added to store the maximum number of replicas and threads available during the whole simulation. They are fixed after initialization. The parameters processor_replica[], task_replica[] and task_multiserver[] should be modified manually in csim_init.h if they are different from the maximum values. If these are found larger than the maximum values, they will be set to the maximum values. 
4. In LQ-CSIM, it uses CSIM storage resource to model the multi threads. One feature of the storage resource in CSIM is that when a storage resource is available, it will be allocated automatically if it has been requested. In CsimSimulator, the total numbers of threads are initialized to the values of task_multiserver_max[] which might be different from the numbers of threads allowed to use currently (task_multiserver []). It will not provide correct results if the storages are just allocated or deallocated without checking. New parameters threads_in_use[] and storage_avail[] are added to monitor the number of threads in use and whether there are available threads to use. Condition checking is added before/after storage allocation and deallocation.

For storage allocation, the code is like

wait(storage_avail[2]);

$\{$;

\}

allocate(1, tasks[2].task);

threads_in_use[2]++;

if (threads_in_use[ 2] < task_multiserver[2])

set(storage_avail[2]);

else

clear(storage_avail[2]);

instead of

allocate(1, tasks[2].task);

For storage deallocation, the code is like

deallocate(1, tasks[2].task);

threads_in_use[2]-;

if (threads_in_use[2] <task_multiserver[2])

set(storage_avail[2]);

else

clear(storage_avail[2]);

instead of 
deallocate(1, tasks[2.task);

In the case of replicas with multithreads, the code for storage allocation is like:

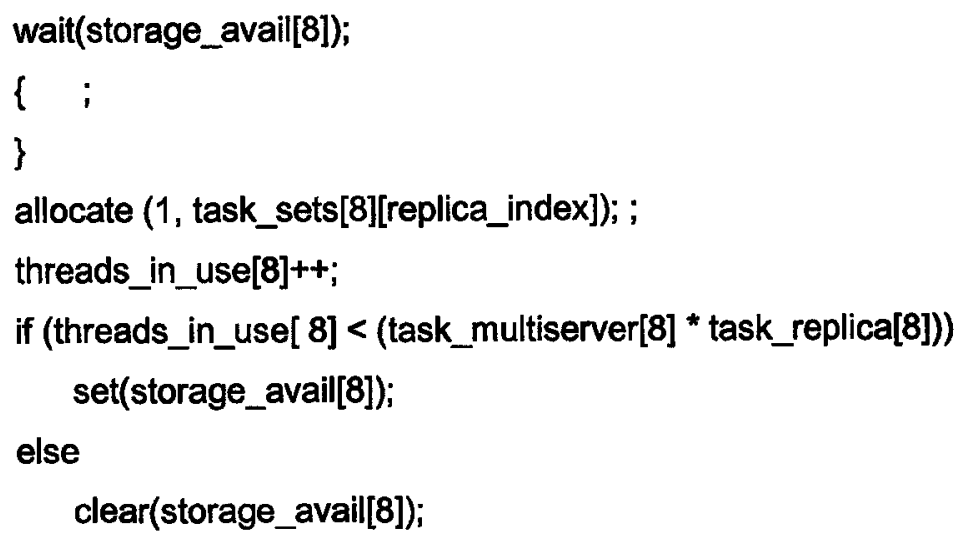

The code for storage deallocation is like:

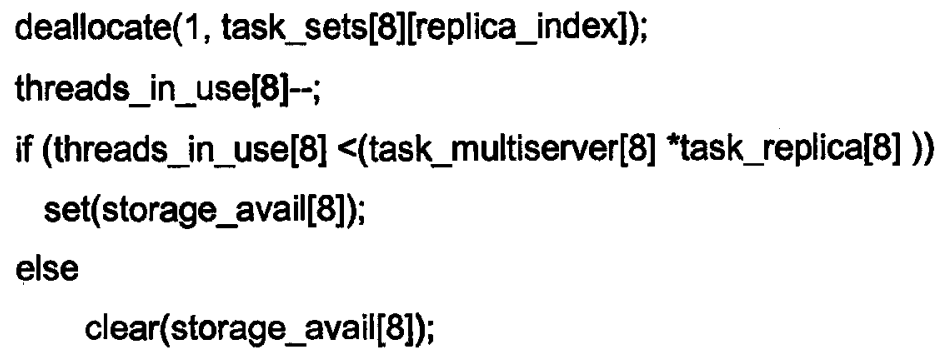

\section{A.3 Data format of the statistic and the communication protocol between CsimSimulator and CsimController}

CsimSimulator provides the following the statistics of the following metrics:

- mean value and variance of the response time of each entry

- throughput of each entry

- CPU utilization of each entry

- task utilization of each entry

- mean value and variance of the service demand of each entry

- CPU utilization of each processor

Other metrics based on tasks and processors could be calculated from those metrics.

The format of the statistics includes the metric name and metric value and a delimiter. Each line has one metric only. The metric name has the format of 
prefix_taskName_entryName or prefix_processorName.

entryName is the name of the specific entry, and taskName is the name of the task where the specific entry belongs to. processorName is the name of the specific processor which applies to the CPU utilization of each processor only. prefix represents the type of metrics.

The list of prefix:
resp_
the prefix of mean value of the response time
resp_var_ the prefix of the variance of the response time
mean the prefix of the mean value of the service demand
variance the prefix of the variance of the service demand
cpu_util the prefix of the CPU utilization
task_util_ the prefix of the task utilization
count_ the prefix of the throughput
proc_cpu the prefix of the CPU utilization of a processor

At each measurement step, the metrics starts with a line contains "Simu_Time" and ends with a line contains

Here is an example of the format:

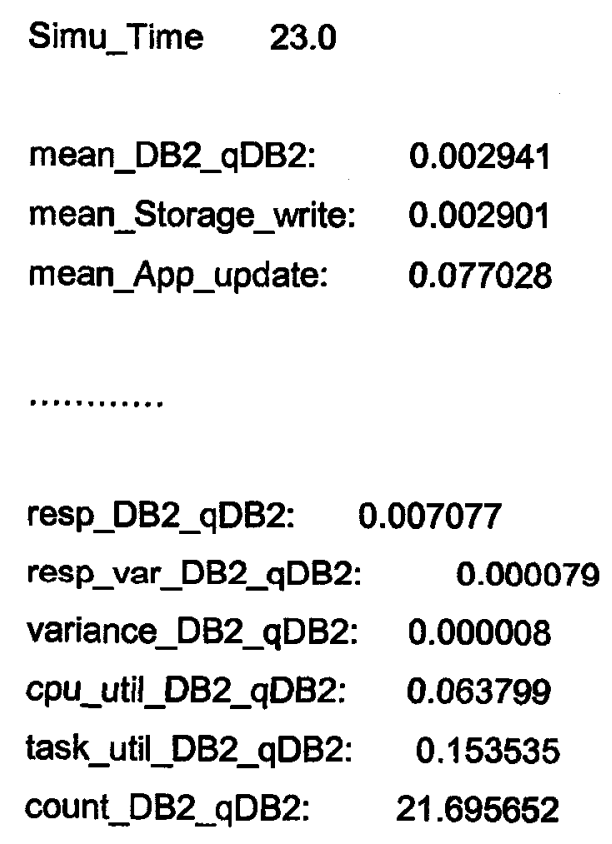




$$
\begin{array}{ll}
\text { proc_cpu_UserProc: } & 18.263458 \\
\text { proc_cpu_DB2Proc: } & 0.177741 \\
\text { proc_cpu_AppProc: } & 0.549562
\end{array}
$$

When the CsimSimulator works in mode 1, the metrics are written to an output file with this format. If the CsimSimulator works in mode 2, the metrics are sent to CsimController with this format. In this case, CsimSimulator receives commands from CsimController as well. The received commands starts with " START $\sim$ " and ends with " END ". A legal command must be a valid task name followed by a character and an integer with delimiter "." and ",". The character has only two valid value: "r" or " $m$ ". The value " $\mathrm{r}$ " indicates that the task should modify the number of replicas while " $\mathrm{m}$ " implies that the task should change the number of threads (or users). The CsimController is responsible to make sure the commands are legal and complete.

Here is an example of the data received from the CsimController:

$\sim$ START

App: $r, \quad 3$

User: $\mathrm{m}, \mathbf{3 0}$

$\sim$ END

"App: $r$, 3" means that the App should change the task replica value. The related processor replica value will be changed as well. "User: $\mathrm{m}, 30$ " means that the task should modify the number of threads to 30 . If "User" is a reference task, it means the "Users" should have a new number of users.

\section{A.4 CsimTracker and CsimController}

Both CsimTracker and CsimController parse the data generated by CsimSimulator and store the metrics in a hash table. The variables measurements[] and trackingParameters [] determine which metrics are measurement data and which parameters needs tracking. CsimTracker/CsimController uses lqns solver to predict the performance metrics. By comparing the predictions with the measurements, it estimates 
the tracking parameters using IEKF algorithm. CsimController tries to optimize the model in addition to the tracking. It suggests a good configuration, transforms the configuration to the control commands and sends the commands to CsimSimulator. CsimSimulator will modify the configuration at runtime and continue. 


\section{Appendix B: LQN Model Files Used in the Thesis}

\section{B.1 The Model Shown in Figure 2.2}

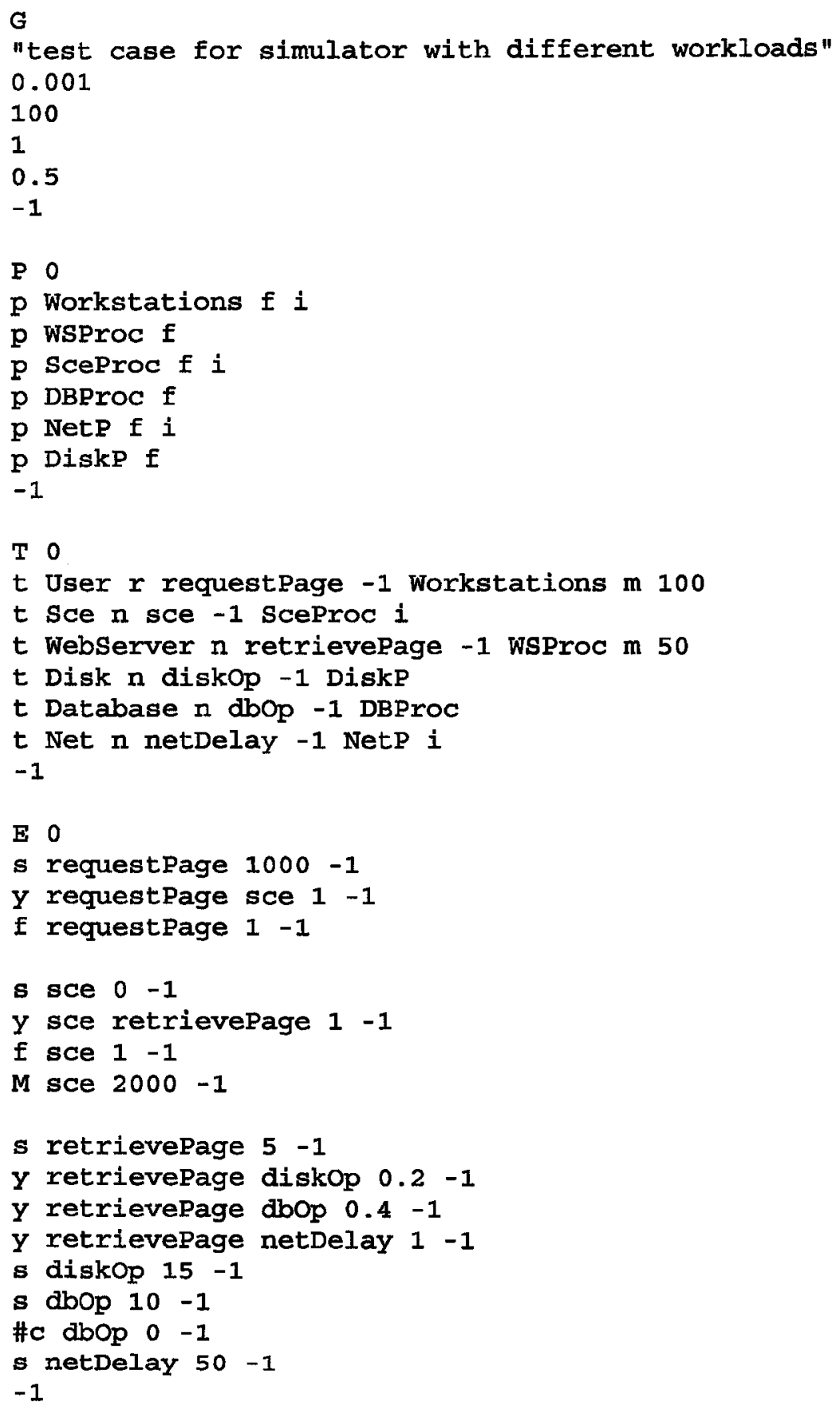




\section{B.2 A Model with Two Classes of Users Shown in Figure 5.31}

G

"a web service system with 2 classes of userg"

0.0001

100

1

0.5

$-1$

P 0

$p$ UserProc $f i$

$p$ ManagerProc $f$ i

p UserPseudoProc $f i$

p ManagerPseudoProc $f i$

p AppProc s

p DB1Proc $s$

p DB2Proc s

p storageProc $\mathrm{f}$

$-1$

T 0

$t$ User $r$ request -1 UserProc m 20

$t$ Manager $r$ manage -1 ManagerProc $m 10$

$t$ UserPseudo $n$ userResponse -1 UserPseudoProc $i$

t ManagerPseudo $n$ managerResponse -1 ManagerPseudoProc $i$

$t$ App in query report update -1 AppProc m 30

$t$ DB1 n $\mathrm{qDB1}$ uDB1 -1 DB1Proc m 5

$t$ DB2 $n$ qDB2 uDB2 -1 DB2Proc $m 5$

$t$ Storage $n$ read write -1 storageProc $m 5$

$-1$

E 0

s request $1-1$

y request userResponse $1-1$

f request $1-1$

s manage $2-1$

y manage managerResponse $1-1$

f manage $1-1$

s userResponse $0-1$

Y userResponse query $0.9-1$

y userResponse report $0.1-1$

s managerResponse $0-1$

Y managerResponse query $0.3-1$

$y$ managerResponse report $0.1-1$

Y managerResponse update $0.6-1$

s query $0.002-1$

s report $0.04-1$

s update $0.008-1$

y query qDBI $2-1$ 


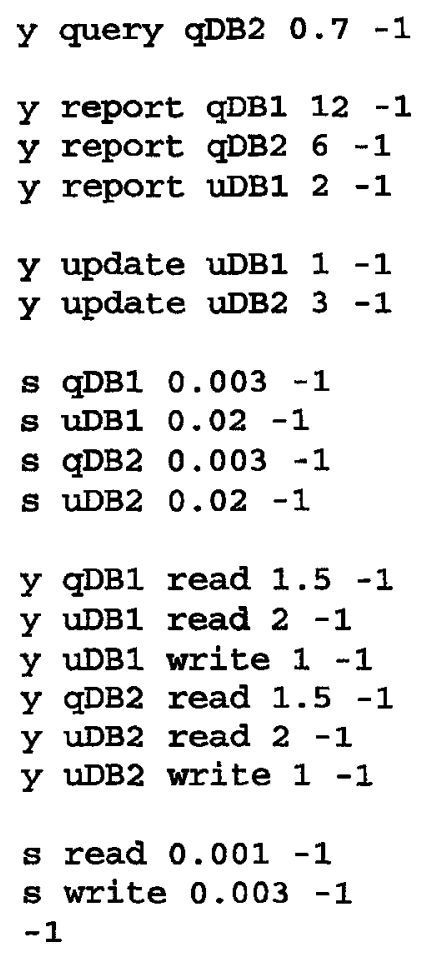

